\title{
Besov and Triebel-Lizorkin Spaces Related to Singular Integrals with Flag Kernels
}

\author{
Dachun YANG \\ School of Mathematical Sciences \\ Beijing Normal University \\ Laboratory of Mathematics and Complex Systems \\ Ministry of Education \\ Beijing 100875 - People's Republic of China \\ dcyang@bnu.edu.cn
}

Received: August 11, 2008

Accepted: January 8, 2009

\begin{abstract}
Let $s_{1}, s_{2} \in(-1,1)$ and $s=\left(s_{1}, s_{2}\right)$. In this paper, the author introduces the Besov space $\dot{B}_{p q}^{s}\left(\mathbb{R}^{2}\right)$ with $p, q \in[1, \infty]$ and the Triebel-Lizorkin space $\dot{F}_{p q}^{s}\left(\mathbb{R}^{2}\right)$ with $p \in(1, \infty)$ and $q \in(1, \infty]$ associated to singular integrals with flag kernels. Some basic properties, including their dual spaces, some equivalent norm characterizations via Littlewood-Paley functions, lifting properties and some embedding theorems, on these spaces are given. Moreover, the author obtains the boundedness of flag singular integrals and fractional integrals on these spaces.
\end{abstract}

Key words: Besov space, Triebel-Lizorkin space, flag singular integral, flag fractional integral, Littlewood-Paley operator, dual space, lifting, embedding.

2000 Mathematics Subject Classification: 42B35, 42B20, 42B25, 46E35.

\section{Introduction}

In order to study the $\square_{b}$-complex on certain quadratic CR submanifolds of $\mathbb{C}^{n}$, Nagel, Ricci, and Stein [6] introduced the notion of singular integrals with flag kernels on $\mathbb{R}^{n}$. Since the flag singular integral is a special case of product singular integrals, the boundedness of flag singular integrals on $L^{p}\left(\mathbb{R}^{n}\right)$ with $p \in(1, \infty)$ is a simple corollary of the boundedness of the corresponding product singular integrals. Recently,

The author is supported by National Science Foundation for Distinguished Young Scholars (No. 10425106) of China. 
Han and Lu in [3] developed a corresponding Hardy space theory for flag singular integrals on $\mathbb{R}^{n}$.

Motivated by [3,6,7], letting $s_{1}, s_{2} \in(-1,1)$ and $s=\left(s_{1}, s_{2}\right)$, in this paper, we introduce the Besov space $\dot{B}_{p q}^{s}\left(\mathbb{R}^{2}\right)$ with $p, q \in[1, \infty]$ and the Triebel-Lizorkin space $\dot{F}_{p q}^{s}\left(\mathbb{R}^{2}\right)$ with $p \in(1, \infty)$ and $q \in(1, \infty]$ associated to singular integrals with flag kernels. Some basic properties, including their dual spaces, some equivalent norm characterizations via Littlewood-Paley functions, lifting properties, and some embedding theorems on these spaces are given. Moreover, we obtain the boundedness of flag singular integrals and fractional integrals on these spaces.

For the simplicity of presentation, we work on flag integrals on $\mathbb{R}^{2}$. However, our method works for more general product Euclidean spaces.

It was proved in $[5,6]$ that flag kernels on $\mathbb{R}^{2}$ are closely connected with product kernels on $\mathbb{R}^{2} \times \mathbb{R}$. We denote any element of $\mathbb{R}^{2} \times \mathbb{R}$ by the 3 -tuple $x=\left(x_{1}, x_{2}, x_{3}\right)$, where $\left(x_{1}, x_{2}\right) \in \mathbb{R}^{2}$ and $x_{3} \in \mathbb{R}$. We endow $\mathbb{R}^{2}$ with the following dilation that for any $\delta>0$ and $x=\left(x_{1}, x_{2}\right) \in \mathbb{R}^{2}, \delta x=\left(\delta x_{1}, \delta^{2} x_{2}\right)$ and the norm that $\|x\|=\left(x_{1}^{2}+\left|x_{2}\right|\right)^{1 / 2}$, which is equivalent to $\left|x_{1}\right|+\left|x_{2}\right|^{1 / 2}$. Obviously, the homogeneous dimension of $\mathbb{R}^{2}$ is 3 .

In order to express the cancellation conditions introduced by Nagel, Ricci, and Stein in [6], we introduce the following terminology. A $k$-normalized bump function on $\mathbb{R}^{n}$ is a $C^{k}$-function supported on the unit ball with $C^{k}$-norm bounded by 1 . It was proved by Nagel, Ricci, and Stein in [6, Remark 2.1.7] that Definitions 1.1 and 1.2 given below are essentially independent of the choice of $k \in \mathbb{N}$. Hence we usually speak of normalized bump functions rather than $k$-normalized bump functions.

In what follows, we denote by $C$ a positive constant which is independent of the main parameters, but it may vary from line to line. Constants with subscripts, such as $C_{1}$, do not change in different occurrences. We also use subscripts to indicate which parameters the constant depends on. Moreover, let $\mathbb{N} \equiv\{1,2, \ldots\}$ and $\mathbb{Z}_{+} \equiv \mathbb{N} \cup\{0\}$.

Definition 1.1. A product kernel on $\mathbb{R}^{2} \times \mathbb{R}$, relative to the given dilations, is a distribution $K$ on $\mathbb{R}^{2} \times \mathbb{R}$ which coincides with a $C^{\infty}$ function away from the coordinate subspace $x_{j}=0$ for $j=1,2,3$ and which satisfies:

(i) (Differential inequalities) For each multi-index $\alpha=\left(\alpha_{1}, \alpha_{2}, \alpha_{3}\right) \in\left(\mathbb{Z}_{+}\right)^{3}$, there exists a positive constant $C_{\alpha}$ so that

$$
\left|\partial_{x_{1}}^{\alpha_{1}} \partial_{x_{2}}^{\alpha_{2}} \partial_{x_{3}}^{\alpha_{3}} K\left(x_{1}, x_{2}, x_{3}\right)\right| \leq C_{\alpha}\left\|\left(x_{1}, x_{2}\right)\right\|^{-3-\alpha_{1}-2 \alpha_{2}}\left|x_{3}\right|^{-1-\alpha_{3}}
$$

away from the coordinate subspaces, where $\partial_{x_{i}}^{\alpha_{i}}=\frac{\partial^{\alpha_{i}}}{\partial x_{i}^{\alpha_{i}}}$.

(ii) (Cancellation conditions)

(a) For each multi-index $\left(\alpha_{1}, \alpha_{2}\right) \in\left(\mathbb{Z}_{+}\right)^{2}$ and any given normalized bump function $\varphi$ on $\mathbb{R}$ and any $\delta>0$, there exists a positive constant $C_{\alpha_{1}, \alpha_{2}}$ 
so that, for all $\left(x_{1}, x_{2}\right) \neq(0,0)$,

$$
\left|\int_{\mathbb{R}} \partial_{x_{1}}^{\alpha_{1}} \partial_{x_{2}}^{\alpha_{2}} K\left(x_{1}, x_{2}, x_{3}\right) \varphi\left(\delta x_{3}\right) d x_{3}\right| \leq C_{\alpha_{1}, \alpha_{2}}\left\|\left(x_{1}, x_{2}\right)\right\|^{-3-\alpha_{1}-2 \alpha_{2}} .
$$

(b) For each $\alpha_{3} \in \mathbb{Z}_{+}$and any given normalized bump function $\varphi$ on $\mathbb{R}^{2}$ and any $\delta>0$, there exists a positive constant $C_{\alpha_{3}}$ so that, for all $x_{3} \neq 0$,

$$
\left|\int_{\mathbb{R}^{2}} \partial_{x_{3}}^{\alpha_{3}} K\left(x_{1}, x_{2}, x_{3}\right) \varphi\left(\delta x_{1}, \delta^{2} x_{2}\right) d x_{1} d x_{2}\right| \leq C_{\alpha_{3}}\left|x_{3}\right|^{-1-\alpha_{3}} .
$$

(c) For any given normalized bump function $\varphi$ on $\mathbb{R}^{2} \times \mathbb{R}$ and any $\delta_{1}, \delta_{2}>0$, there exists a positive constant $C$ so that

$$
\left|\int_{\mathbb{R}^{2} \times \mathbb{R}} K\left(x_{1}, x_{2}, x_{3}\right) \varphi\left(\delta_{1} x_{1}, \delta_{1}^{2} x_{2}, \delta_{2} x_{3}\right) d x_{1} d x_{2} d x_{3}\right| \leq C .
$$

The following definition of flag kernels is just a special case of flag kernels in [6].

Definition 1.2. A flag kernel on $\mathbb{R}^{2}$, relative to the given dilations, is a distribution $K$ on $\mathbb{R}^{2}$ which coincides with a $C^{\infty}$ function away from the coordinate subspace $x_{1}=0$ and which satisfies:

(i) (Differential inequalities) For each $\alpha=\left(\alpha_{1}, \alpha_{2}\right) \in\left(\mathbb{Z}_{+}\right)^{2}$, there exists a positive constant $C_{\alpha}$ so that, for all $x_{1} \neq 0$,

$$
\left|\partial_{x_{1}}^{\alpha_{1}} \partial_{x_{2}}^{\alpha_{2}} K\left(x_{1}, x_{2}\right)\right| \leq C_{\alpha}\left|x_{1}\right|^{-1-\alpha_{1}}\left\|\left(x_{1}, x_{2}\right)\right\|^{-2-2 \alpha_{2}} .
$$

(ii) (Cancellation conditions)

(a) For any given normalized bump function $\varphi$ on $\mathbb{R}$, any $\alpha_{1} \in \mathbb{Z}_{+}$, and any $\delta>0$, there exists a positive constant $C_{\alpha_{1}}$ so that, for all $x_{1} \neq 0$,

$$
\left|\int_{\mathbb{R}} \partial_{x_{1}}^{\alpha_{1}} K\left(x_{1}, x_{2}\right) \varphi\left(\delta x_{2}\right) d x_{2}\right| \leq C_{\alpha_{1}}\left|x_{1}\right|^{-1-\alpha_{1}} .
$$

(b) For any given normalized bump function $\varphi$ on $\mathbb{R}$, any $\alpha_{2} \in \mathbb{Z}_{+}$, and any $\delta>0$, there exists a positive constant $C_{\alpha_{2}}$ so that for all $x_{2} \neq 0$,

$$
\left|\int_{\mathbb{R}} \partial_{x_{2}}^{\alpha_{2}} K\left(x_{1}, x_{2}\right) \varphi\left(\delta x_{1}\right) d x_{1}\right| \leq C_{\alpha_{2}}\left|x_{2}\right|^{-1-\alpha_{2}} .
$$

(c) For any given normalized bump function $\varphi$ on $\mathbb{R}^{2}$, and any $\delta_{1}, \delta_{2}>0$, there exists a positive constant $C$ so that

$$
\left|\int_{\mathbb{R}^{2}} K\left(x_{1}, x_{2}\right) \varphi\left(\delta_{1} x_{1}, \delta_{2} x_{2}\right) d x_{1} d x_{2}\right| \leq C .
$$


Remark 1.3. It was pointed by Nagel, Ricci, and Stein in [6] that the single normalized bump function in Definitions 1.1 and 1.2 (ii)-(c) can be replaced by the tensor product of normalized bump functions on $\mathbb{R}^{2}$ and $\mathbb{R}$.

The following proposition is completely similar to Proposition 3.2 and Lemma 4.5 in [5], which reveals the relation between the product kernel and the flag kernel.

Proposition 1.4. Let $K^{\sharp}$ be an integrable function on $\mathbb{R}^{2} \times \mathbb{R}$ which is a product kernel as in Definition 1.1. Then, for $\left(x_{1}, x_{2}\right) \in \mathbb{R}^{2}$,

$$
K\left(x_{1}, x_{2}\right)=\int_{\mathbb{R}} K^{\sharp}\left(x_{1}, x_{2}-x_{3}, x_{3}\right) d x_{3}
$$

is a flag singular kernel on $\mathbb{R}^{2}$.

Conversely, given $K \in L^{1}\left(\mathbb{R}^{2}\right)$ which is a flag kernel as in Definition 1.2, then

$$
K^{\sharp}\left(x_{1}, x_{2}, x_{3}\right)=\frac{1}{\left|x_{1}\right|^{2}} \chi\left(\frac{x_{2}}{\left|x_{1}\right|^{2}}\right) K\left(x_{1}, x_{2}+x_{3}\right),
$$

where $\chi$ is a non-negative smooth function supported on $[1 / 2,1]$ such that $\int_{1 / 2}^{1} \chi(t) d t=1$, is an integrable product kernel on $\mathbb{R}^{2} \times \mathbb{R}$ such that (1) holds.

The organization of this paper is as follows. In section 2, we first establish some Calderón reproducing formulae (see Lemma 2.3), whose dyadic version (see Lemma 2.4) are essentially included in [3]. However, in this paper, we use a slightly different way from [3] to define the topology of $\mathcal{S}_{\infty, F}\left(\mathbb{R}^{2}\right)$; see Definition 2.1 below and Definition 1.6 in [3]. Let $s_{1}, s_{2} \in(-1,1)$ and $s=\left(s_{1}, s_{2}\right)$. With a special choice of approximations of the identity associated to flag kernels (see (1.3) of [3]), we then introduce the norms of $\|\cdot\|_{\dot{B}_{p q}^{s}\left(\mathbb{R}^{2}\right)}$ and $\|\cdot\|_{\dot{F}_{p q}^{s}\left(\mathbb{R}^{2}\right)}$ in Definition 2.5, and using the Calderón reproducing formulae, we prove in Theorem 2.6 that these norms are independent of the choice of the approximations of the identity associated to flag kernels. Then we introduce the Besov space $\dot{B}_{p q}^{s}\left(\mathbb{R}^{2}\right)$ and the Triebel-Lizorkin space $\dot{F}_{p q}^{s}\left(\mathbb{R}^{2}\right)$ in Definition 2.7. Some basic properties including dual spaces of these spaces are presented in Propositions 2.9, 2.10, and 2.11. In Theorems 2.8, 2.14 and Corollary 2.22 , we establish some equivalent norm characterizations of these spaces including various Littlewood-Paley functions. We remark that Corollary 2.22 clearly reveals the difference between $\dot{B}_{p q}^{s}\left(\mathbb{R}^{2}\right)$ and $\dot{F}_{p q}^{s}\left(\mathbb{R}^{2}\right)$ with the classical product Besov and Triebel-Lizorkin spaces in [7].

The boundedness of flag singular integrals on $\dot{B}_{p q}^{s}\left(\mathbb{R}^{2}\right)$ and $\dot{F}_{p q}^{s}\left(\mathbb{R}^{2}\right)$ is given in Theorem 3.1 and the lifting properties of these spaces via Riesz potential operators is presented in Theorem 4.6.

Finally, in Theorems 5.1 and 5.2, we establish some embedding theorems on $\dot{B}_{p q}^{s}\left(\mathbb{R}^{2}\right)$ and $\dot{F}_{p q}^{s}\left(\mathbb{R}^{2}\right)$. The boundedness of flag fractional integrals is given in Theorem 5.4. 


\section{Besov and Triebel-Lizorkin spaces on $\mathbb{R}^{2}$}

We first introduce the Calderón reproducing formulae. To this end, we need to introduce some spaces of distributions; see [3].

Definition 2.1. A Schwartz function $f \in \mathcal{S}\left(\mathbb{R}^{2}\right)$ is said to belong to the space of test functions, $\mathcal{S}_{\infty, F}\left(\mathbb{R}^{2}\right)$, related to flag singular integrals, if there exists a Schwartz function $f^{\sharp} \in \mathcal{S}\left(\mathbb{R}^{2} \times \mathbb{R}\right)$ such that, for all $\left(x_{1}, x_{2}\right) \in \mathbb{R}^{2}$,

$$
f\left(x_{1}, x_{2}\right)=\int_{\mathbb{R}} f^{\sharp}\left(x_{1}, x_{2}-x_{3}, x_{3}\right) d x_{3},
$$

where $f^{\sharp}$ satisfies the following conditions: for all $x_{3} \in \mathbb{R}$ and $\left(\alpha_{1}, \alpha_{2}\right) \in\left(\mathbb{Z}_{+}\right)^{2}$,

$$
\int_{\mathbb{R}^{2}} f^{\sharp}\left(x_{1}, x_{2}, x_{3}\right) x_{1}^{\alpha_{1}} x_{2}^{\alpha_{2}} d x_{1} d x_{2}=0,
$$

and for all $\left(x_{1}, x_{2}\right) \in \mathbb{R}^{2}$ and $\alpha_{3} \in \mathbb{Z}_{+}$,

$$
\int_{\mathbb{R}} f^{\sharp}\left(x_{1}, x_{2}, x_{3}\right) x_{3}^{\alpha_{3}} d x_{3}=0 .
$$

We endow $\mathcal{S}_{\infty, F}\left(\mathbb{R}^{2}\right)$ with the same topology as $\mathcal{S}\left(\mathbb{R}^{2}\right)$, and we denote its dual space by $\mathcal{S}_{\infty, F}\left(\mathbb{R}^{2}\right)^{\prime}$.

Remark 2.2. It is easy to see that the space $\mathcal{S}_{\infty, F}\left(\mathbb{R}^{2}\right)$ is a closed subspace of $\mathcal{S}\left(\mathbb{R}^{2}\right)$, and if $f \in \mathcal{S}_{\infty, F}\left(\mathbb{R}^{2}\right)$ then, for all $\alpha_{2} \in \mathbb{Z}_{+}$,

$$
\int_{\mathbb{R}} f\left(x_{1}, x_{2}\right) x_{2}^{\alpha_{2}} d x_{2}=0 .
$$

Let $\mathcal{P}_{x_{2}}(\mathbb{R})$ be the set of all polynomials on $\mathbb{R}$ in variable $x_{2}$. Then, one can easily see that if $f \in \mathcal{S}_{\infty, F}\left(\mathbb{R}^{2}\right)^{\prime}, h \in \mathcal{P}_{x_{2}}(\mathbb{R})$, and $g \in \mathcal{S}_{\infty, F}\left(\mathbb{R}^{2}\right)$, then $\langle f, g\rangle=\langle f, g+h\rangle$, namely, $\mathcal{S}_{\infty}\left(\mathbb{R}^{2}\right)^{\prime} / \mathcal{P}_{x_{2}}(\mathbb{R}) \subset \mathcal{S}_{\infty, F}\left(\mathbb{R}^{2}\right)^{\prime}$.

We now establish the following Calderón reproducing formulae by a method essentially similar to that of Theorem 3 in the appendix of [2] and a dual argument; see also [3].

Lemma 2.3. Let $\psi^{(1)} \in \mathcal{S}\left(\mathbb{R}^{2}\right)$ with $\int_{\mathbb{R}^{2}} \psi^{(1)}\left(x_{1}, x_{2}\right) d x_{1} d x_{2}=0$ and $\psi^{(2)} \in \mathcal{S}(\mathbb{R})$ with $\int_{\mathbb{R}} \psi^{(2)}\left(x_{3}\right) d x_{3}=0$ satisfy the following admissible conditions: for all $\left(\xi_{1}, \xi_{2}\right) \in \mathbb{R}^{2}$ and $\left(\xi_{1}, \xi_{2}\right) \neq 0$,

$$
\int_{0}^{\infty}\left|\hat{\psi}^{(1)}\left(t \xi_{1}, t^{2} \xi_{2}\right)\right|^{2} \frac{d t}{t}=1,
$$


and, for all $\eta \in \mathbb{R}$ and $\eta \neq 0$,

$$
\int_{0}^{\infty}\left|\hat{\psi}^{(2)}(t \eta)\right|^{2} \frac{d t}{t}=1
$$

For $t_{1}, t_{2}>0$ and $x_{1}, x_{2} \in \mathbb{R}$, let

and

$$
\psi_{t_{1}}^{(1)}\left(x_{1}, x_{2}\right)=\frac{1}{t_{1}^{3}} \psi^{(1)}\left(\frac{x_{1}}{t_{1}}, \frac{x_{2}}{t_{1}^{2}}\right), \quad \psi_{t_{2}}^{(2)}\left(x_{2}\right)=\frac{1}{t_{2}} \psi^{(2)}\left(\frac{x_{2}}{t_{2}}\right),
$$

$$
\psi_{t_{1} t_{2}}\left(x_{1}, x_{2}\right)=\int_{\mathbb{R}} \psi_{t_{1}}^{(1)}\left(x_{1}, x_{2}-x_{2}^{\prime}\right) \psi_{t_{2}}^{(2)}\left(x_{2}^{\prime}\right) d x_{2}^{\prime} .
$$

Then the identity

$$
f\left(x_{1}, x_{2}\right)=\int_{0}^{\infty} \int_{0}^{\infty} \psi_{t_{1} t_{2}} * \psi_{t_{1} t_{2}} * f\left(x_{1}, x_{2}\right) \frac{d t_{1}}{t_{1}} \frac{d t_{2}}{t_{2}}
$$

holds in $L^{2}\left(\mathbb{R}^{2}\right), \mathcal{S}_{\infty, F}\left(\mathbb{R}^{2}\right)$, and $\mathcal{S}_{\infty, F}\left(\mathbb{R}^{2}\right)^{\prime}$.

Proof. From the Plancherel principle and the assumptions of the lemma, it is easy to see that (2) holds in $L^{2}\left(\mathbb{R}^{2}\right)$. The fact that (2) holds in $\mathcal{S}_{\infty, F}\left(\mathbb{R}^{2}\right)$ and a dual argument show that (2) also holds in $\mathcal{S}_{\infty, F}\left(\mathbb{R}^{2}\right)^{\prime}$. Thus, to finish the proof of Lemma 2.3 , we only need to show that (2) holds in $\mathcal{S}_{\infty, F}\left(\mathbb{R}^{2}\right)$. To do so, for $f \in \mathcal{S}_{\infty, F}\left(\mathbb{R}^{2}\right), \epsilon_{i}>0$, and $\delta_{i}>0$ with $i=1,2$ and $\left(x_{1}, x_{2}\right) \in \mathbb{R}^{2}$, set

$$
f_{\epsilon_{1}, \epsilon_{2}, \delta_{1}, \delta_{2}}\left(x_{1}, x_{2}\right)=\int_{\epsilon_{1}}^{\delta_{1}} \int_{\epsilon_{2}}^{\delta_{1}} \psi_{t_{1} t_{2}} * \psi_{t_{1} t_{2}} * f\left(x_{1}, x_{2}\right) \frac{d t_{1}}{t_{1}} \frac{d t_{2}}{t_{2}} .
$$

We only need to show that for any fixed $N \in \mathbb{Z}_{+}$and all $\left(x_{1}, x_{2}\right) \in \mathbb{R}^{2}$, there exists a positive constant $C=C_{f, \psi^{(1)}, \psi^{(2)}, N}$ such that

$$
\left|f\left(x_{1}, x_{2}\right)-f_{\epsilon_{1}, \epsilon_{2}, \delta_{1}, \delta_{2}}\left(x_{1}, x_{2}\right)\right| \leq C\left(\epsilon_{1}+\frac{1}{\delta_{1}}\right)\left(\epsilon_{2}+\frac{1}{\delta_{2}}\right)\left(1+\left\|\left(x_{1}, x_{2}\right)\right\|\right)^{-N} .
$$

Obviously, we may assume that $0<\epsilon_{i}<1<\delta_{i}$ for $i=1,2$ in (3). To prove (3), we write

$$
\begin{aligned}
f\left(x_{1}, x_{2}\right)-f_{\epsilon_{1}, \epsilon_{2}, \delta_{1}, \delta_{2}}\left(x_{1}, x_{2}\right)= & \int_{0}^{\epsilon_{1}} \int_{0}^{\infty} \psi_{t_{1} t_{2}} * \psi_{t_{1} t_{2}} * f\left(x_{1}, x_{2}\right) \frac{d t_{1}}{t_{1}} \frac{d t_{2}}{t_{2}} \\
& +\int_{\epsilon_{1}}^{\delta_{1}} \int_{0}^{\epsilon_{2}} \cdots+\int_{\epsilon_{1}}^{\delta_{1}} \int_{\delta_{2}}^{\infty} \cdots+\int_{\delta_{1}}^{\infty} \int_{0}^{\infty} \cdots \\
= & H_{1}+H_{2}+H_{3} .
\end{aligned}
$$


We only estimate $H_{1}$. The same technique also works for $H_{2}$ and $H_{3}$. To estimate $H_{1}$, we further decompose it into

$$
H_{1}=\int_{0}^{\epsilon_{1}} \int_{0}^{1} \psi_{t_{1} t_{2}} * \psi_{t_{1} t_{2}} * f\left(x_{1}, x_{2}\right) \frac{d t_{1}}{t_{1}} \frac{d t_{2}}{t_{2}}+\int_{0}^{\epsilon_{1}} \int_{1}^{\infty} \cdots=H_{1}^{1}+H_{1}^{2} .
$$

Let $\varphi^{(i)}=\psi^{(i)} * \psi^{(i)}$ for $i=1,2$ and $\varphi_{t_{1} t_{2}}=\varphi_{t_{1}}^{(1)} * \varphi_{t_{2}}^{(2)}$. Then it is easy to see that $\varphi_{t_{1} t_{2}}=\psi_{t_{1} t_{2}} * \psi_{t_{1} t_{2}}$. Since $f \in \mathcal{S}_{\infty, F}\left(\mathbb{R}^{2}\right)$, by Definition 2.1 , there exists a function $f^{\sharp}$ as in Definition 2.1 such that, for all $\left(x_{1}, x_{2}\right) \in \mathbb{R}^{2}$,

$$
f\left(x_{1}, x_{2}\right)=\int_{\mathbb{R}} f^{\sharp}\left(x_{1}, x_{2}-x_{3}, x_{3}\right) d x_{3} .
$$

Using this fact, we have

$$
\begin{array}{rl}
\psi_{t_{1} t_{2}} & * \psi_{t_{1} t_{2}} * f\left(x_{1}, x_{2}\right) \\
& =\int_{\mathbb{R}} \int_{\mathbb{R}^{2}} \int_{\mathbb{R}} \varphi_{t_{1}}^{(1)}\left(x_{1}-y_{1}, x_{2}-y_{2}-z\right) \varphi_{t_{2}}^{(2)}\left(z-y_{3}\right) f^{\sharp}\left(y_{1}, y_{2}, y_{3}\right) d z d y_{1} d y_{2} d y_{3} .
\end{array}
$$

By the vanishing moments of $\varphi^{(1)}$ and $\varphi^{(2)}$, we further write

$$
\begin{aligned}
\psi_{t_{1} t_{2}} * & \psi_{t_{1} t_{2}} * f\left(x_{1}, x_{2}\right) \\
= & \int_{\mathbb{R}} \int_{\left|z-y_{3}\right| \leq|z| / 2} \int_{\left\|\left(x_{1}-y_{1}, x_{2}-y_{2}-z\right)\right\| \leq\left\|\left(x_{1}, x_{2}-z\right)\right\| / 2} \varphi_{t_{1}}^{(1)}\left(x_{1}-y_{1}, x_{2}-y_{2}-z\right) \\
& \times \varphi_{t_{2}}^{(2)}\left(z-y_{3}\right)\left\{\left[f^{\sharp}\left(y_{1}, y_{2}, y_{3}\right)-f^{\sharp}\left(x_{1}, x_{2}-z, y_{3}\right)\right]\right. \\
& \left.\quad-\left[f^{\sharp}\left(y_{1}, y_{2}, z\right)-f^{\sharp}\left(x_{1}, x_{2}-z, z\right)\right]\right\} d y_{1} d y_{2} d y_{3} d z \\
& +\int_{\mathbb{R}} \int_{\left|z-y_{3}\right|>|z| / 2} \int_{\left\|\left(x_{1}-y_{1}, x_{2}-y_{2}-z\right)\right\| \leq\left\|\left(x_{1}, x_{2}-z\right)\right\| / 2} \cdots \\
& +\int_{\mathbb{R}} \int_{\left|z-y_{3}\right| \leq|z| / 2} \int_{\left\|\left(x_{1}-y_{1}, x_{2}-y_{2}-z\right)\right\|>\left\|\left(x_{1}, x_{2}-z\right)\right\| / 2} \cdots \\
& +\int_{\mathbb{R}} \int_{\left|z-y_{3}\right|>|z| / 2} \int_{\left\|\left(x_{1}-y_{1}, x_{2}-y_{2}-z\right)\right\|>\left\|\left(x_{1}, x_{2}-z\right)\right\| / 2} \cdots=I_{1}+I_{2}+I_{3}+I_{4} .
\end{aligned}
$$

We denote the corresponding terms of $H_{1}^{1}$ to $I_{i}$, respectively, by $H_{1, i}^{1}$, where $i=1,2,3,4$, and by similarity we only estimate $H_{1,1}^{1}$ and $H_{1,2}^{1}$. By the mean value 
theorem, we have

$$
\begin{aligned}
\left|I_{1}\right| \leq C & \int_{\mathbb{R}} \int_{\mathbb{R}} \int_{\mathbb{R}^{2}}\left|\varphi_{t_{1}}^{(1)}\left(x_{1}-y_{1}, x_{2}-y_{2}-z\right)\right| \\
\times & {\left[\frac{\left|x_{1}-y_{1}\right|}{\left(1+\left\|\left(x_{1}, x_{2}-z\right)\right\|\right)^{N+2}}+\frac{\left|x_{2}-y_{2}-z\right|^{1 / 2}}{\left(1+\left\|\left(x_{1}, x_{2}-z\right)\right\|\right)^{N+2}}\right] } \\
& \times\left|\varphi_{t_{2}}^{(2)}\left(z-y_{3}\right)\right|\left|z-y_{3}\right| \frac{1}{(1+|z|)^{N}} d y_{1} d y_{2} d y_{3} d z \\
\leq & C t_{1} t_{2} \int_{\mathbb{R}} \frac{1}{\left(1+\left\|\left(x_{1}, x_{2}-z\right)\right\|\right)^{N+2}} \frac{1}{(1+|z|)^{N}} d z \leq C t_{1} t_{2} \frac{1}{\left(1+\left\|\left(x_{1}, x_{2}\right)\right\|\right)^{N}} .
\end{aligned}
$$

From this, we can easily deduce a desired estimate for $H_{1,1}^{1}$. Similarly, we have

$$
\begin{aligned}
\left|I_{2}\right| \leq & C t_{1} \int_{\mathbb{R}} \int_{\left|z-y_{3}\right|>|z| / 2}\left|\varphi_{t_{2}}^{(2)}\left(z-y_{3}\right)\right|\left|y_{3}-z\right| \\
& \quad \times \frac{1}{\left(1+\left\|\left(x_{1}, x_{2}-z\right)\right\|\right)^{N+2}} d y_{3} d z \\
\leq & C t_{1} t_{2} \int_{\mathbb{R}} \frac{1}{\left(1+\left\|\left(x_{1}, x_{2}-z\right)\right\|\right)^{N+2}} \frac{1}{(1+|z|)^{N}} d z \\
\leq & C t_{1} t_{2} \frac{1}{\left(1+\left\|\left(x_{1}, x_{2}\right)\right\|\right)^{N}},
\end{aligned}
$$

which gives a desired estimate of $H_{1,2}^{1}$.

In what follows, we denote by $\lfloor a\rfloor$ the integer no more than $a \in \mathbb{R}$. To estimate $H_{1}^{2}$, by the vanishing moments of $\varphi^{(1)}$ and $f^{\sharp}$, we write

$$
\begin{array}{rl}
\psi_{t_{1} t_{2}} * \psi_{t_{1} t_{2}} & * f\left(x_{1}, x_{2}\right) \\
= & \int_{\mathbb{R}} \int_{\left|y_{3}\right| \leq|z| / 2} \int_{\left\|\left(x_{1}-y_{1}, x_{2}-y_{2}-z\right)\right\| \leq\left\|\left(x_{1}, x_{2}-z\right)\right\| / 2} \varphi_{t_{1}}^{(1)}\left(x_{1}-y_{1}, x_{2}-y_{2}-z\right) \\
& \times\left[\varphi_{t_{2}}^{(2)}\left(z-y_{3}\right)-\sum_{\gamma=0}^{\lfloor N / 2\rfloor}\left(-y_{3}\right)^{\gamma} \frac{d^{\gamma}}{d z^{\gamma}} \varphi_{t_{2}}^{(2)}(z)\right] \\
& \times\left[f^{\sharp}\left(y_{1}, y_{2}, y_{3}\right)-f^{\sharp}\left(x_{1}, x_{2}-z, y_{3}\right)\right] d y_{1} d y_{2} d y_{3} d z \\
& +\int_{\mathbb{R}} \int_{\left|y_{3}\right|>|z| / 2} \int_{\left\|\left(x_{1}-y_{1}, x_{2}-y_{2}-z\right)\right\| \leq\left\|\left(x_{1}, x_{2}-z\right)\right\| / 2} \cdots \\
& +\int_{\mathbb{R}} \int_{\left|y_{3}\right| \leq|z| / 2} \int_{\left\|\left(x_{1}-y_{1}, x_{2}-y_{2}-z\right)\right\|>\left\|\left(x_{1}, x_{2}-z\right)\right\| / 2} \cdots \\
& +\int_{\mathbb{R}} \int_{\left|y_{3}\right|>|z| / 2} \int_{\left\|\left(x_{1}-y_{1}, x_{2}-y_{2}-z\right)\right\|>\left\|\left(x_{1}, x_{2}-z\right)\right\| / 2} \cdots=J_{1}+J_{2}+J_{3}+J_{4},
\end{array}
$$


and we also denote the corresponding terms of $H_{1}^{2}$ to $J_{i}$, respectively, by $H_{1, i}^{2}$, where $i=1,2,3,4$. By similarity, we only estimate $H_{1,1}^{2}$. The Taylor expansion theorem yields

$$
\begin{aligned}
\left|J_{1}\right| & \leq C \int_{\mathbb{R}} \frac{t_{1}}{\left(1+\left\|\left(x_{1}, x_{2}-z\right)\right\|\right)^{N+2}} \frac{1}{t_{2}} \frac{1}{\left(t_{2}+|z|\right)^{\lfloor N / 2\rfloor+1}} d z \\
& \leq C \frac{t_{1}}{\left(1+\left\|\left(x_{1}, x_{2}\right)\right\|\right)^{N+2}} \frac{1}{t_{2}^{\lfloor N / 2\rfloor+1}}+C \frac{t_{1}}{\left(1+\left|x_{1}\right|\right)^{N}} \frac{1}{t_{2}} \frac{1}{\left(t_{2}+\left|x_{2}\right|\right)^{\lfloor N / 2\rfloor+1}},
\end{aligned}
$$

which yields a desired estimate for $H_{1,1}^{2}$. This finishes the proof of Lemma 2.3.

Similarly, we have a 'discrete' version of Lemma 2.3 and we omit the details of its proof; see [3].

Lemma 2.4. Let $\psi^{(1)} \in \mathcal{S}\left(\mathbb{R}^{2}\right)$ with $\int_{\mathbb{R}^{2}} \psi^{(1)}\left(x_{1}, x_{2}\right) d x_{1} d x_{2}=0$ and $\psi^{(2)} \in \mathcal{S}(\mathbb{R})$ with $\int_{\mathbb{R}} \psi^{(2)}\left(x_{3}\right) d x_{3}=0$ satisfy the following admissible conditions that for all $\left(\xi_{1}, \xi_{2}\right) \in \mathbb{R}^{2}$ and $\left(\xi_{1}, \xi_{2}\right) \neq 0$,

$$
\sum_{k_{1}=-\infty}^{\infty}\left|\hat{\psi}^{(1)}\left(2^{-k_{1}} \xi_{1}, 2^{-2 k_{1}} \xi_{2}\right)\right|^{2}=1
$$

and for all $\eta \in \mathbb{R}$ and $\eta \neq 0, \sum_{k_{2}=-\infty}^{\infty}\left|\hat{\psi}^{(2)}\left(2^{-k_{2}} \eta\right)\right|^{2}=1$. For all $k_{1}, k_{2} \in \mathbb{Z}$ and $x_{1}, x_{2} \in \mathbb{R}$, let $\psi_{k_{1}}^{(1)}\left(x_{1}, x_{2}\right)=2^{3 k_{1}} \psi^{(1)}\left(2^{k_{1}} x_{1}, 2^{2 k_{1}} x_{2}\right), \psi_{k_{2}}^{(2)}\left(x_{2}\right)=2^{k_{2}} \psi^{(2)}\left(2^{k_{2}} x_{2}\right)$, and

$$
\psi_{k_{1} k_{2}}\left(x_{1}, x_{2}\right)=\int_{\mathbb{R}} \psi_{k_{1}}^{(1)}\left(x_{1}, x_{2}-x_{2}^{\prime}\right) \psi_{k_{2}}^{(2)}\left(x_{2}^{\prime}\right) d x_{2}^{\prime} .
$$

Then the identity

$$
f\left(x_{1}, x_{2}\right)=\sum_{k_{1}=-\infty}^{\infty} \sum_{k_{2}=-\infty}^{\infty} \psi_{k_{1} k_{2}} * \psi_{k_{1} k_{2}} * f\left(x_{1}, x_{2}\right)
$$

holds in $L^{2}\left(\mathbb{R}^{2}\right), \mathcal{S}_{\infty, F}\left(\mathbb{R}^{2}\right)$, and $\mathcal{S}_{\infty, F}\left(\mathbb{R}^{2}\right)^{\prime}$.

We now introduce the norms $\|\cdot\|_{\dot{B}_{p q}^{s}\left(\mathbb{R}^{2}\right)}$ and $\|\cdot\|_{\dot{F}_{p q}^{s}\left(\mathbb{R}^{2}\right)}$ for $f \in \mathcal{S}_{\infty, F}\left(\mathbb{R}^{2}\right)^{\prime}$ and using Lemma 2.3, we prove that they are independent of choices of $\psi^{(1)}$ and $\psi^{(2)}$.

Definition 2.5. Let $\psi_{t_{1} t_{2}}$ be the same as in Lemma 2.3 and $s_{1}, s_{2} \in \mathbb{R}$. For $f \in \mathcal{S}_{\infty, F}\left(\mathbb{R}^{2}\right)^{\prime}$, define

$$
\|f\|_{\dot{B}_{p q}^{s}\left(\mathbb{R}^{2}\right)}=\left\{\int_{0}^{\infty} \int_{0}^{\infty} t_{1}^{-s_{1} q} t_{2}^{-s_{2} q}\left\|\psi_{t_{1} t_{2}} * f\right\|_{L^{p}\left(\mathbb{R}^{2}\right)}^{q} \frac{d t_{1}}{t_{1}} \frac{d t_{2}}{t_{2}}\right\}^{1 / q}
$$


for $p, q \in[1, \infty]$, and

$$
\|f\|_{\dot{F}_{p q}^{s}\left(\mathbb{R}^{2}\right)}=\left\|\left\{\int_{0}^{\infty} \int_{0}^{\infty} t_{1}^{-s_{1} q} t_{2}^{-s_{2} q}\left|\psi_{t_{1} t_{2}} * f\right|^{q} \frac{d t_{1}}{t_{1}} \frac{d t_{2}}{t_{2}}\right\}^{1 / q}\right\|_{L^{p}\left(\mathbb{R}^{2}\right)}
$$

for $p \in(1, \infty)$ and $q \in(1, \infty]$, where the usual modifications are made when $p=\infty$ or $q=\infty$.

We recall the definition of the strong maximal function: for any $f \in L_{\text {loc }}^{1}\left(\mathbb{R}^{2}\right)$ and all $\left(x_{1}, x_{2}\right) \in \mathbb{R}^{2}$,

$$
M_{s}(f)\left(x_{1}, x_{2}\right)=\sup _{\substack{\left(x_{1}, x_{2}\right) \in R \\ R \text { rectangle }}} \frac{1}{|R|} \int_{R}\left|f\left(y_{1}, y_{2}\right)\right| d y_{1} d y_{2} .
$$

Theorem 2.6. Let $s_{1}, s_{2} \in(-1,1)$ and $s=\left(s_{1}, s_{2}\right)$. The norm $\|\cdot\|_{\dot{B}_{p q}^{s}\left(\mathbb{R}^{2}\right)}$ with $p, q \in[1, \infty]$ and the norm $\|\cdot\|_{\dot{F}_{p q}^{s}\left(\mathbb{R}^{2}\right)}$ with $p \in(1, \infty)$ and $q \in(1, \infty]$ are independent of the choices of $\psi^{(i)}$ for $i=1,2$.

Proof. Let $\tilde{\psi}^{(i)}$ be some other functions satisfying the same conditions as $\psi^{(i)}$ for $i=1,2$. We denote the corresponding norms, respectively, by $\|\cdot\|_{0} \dot{B}_{p q}^{s}\left(\mathbb{R}^{2}\right)$ and $\|\cdot\|_{0} \dot{F}_{p q}^{s}\left(\mathbb{R}^{2}\right)$ and now prove that there exists a positive constant $C$ such that, for all $f \in \mathcal{S}_{\infty, F}\left(\mathbb{R}^{2}\right)^{\prime}$,

$$
\|f\|_{\dot{B}_{p q}^{s}}\left(\mathbb{R}^{2}\right) \leq C\|f\|_{\dot{B}_{p q}^{s}\left(\mathbb{R}^{2}\right)}
$$

and

$$
\|f\|_{0 \dot{F}_{p q}^{s}\left(\mathbb{R}^{2}\right)} \leq C\|f\|_{\dot{F}_{p q}^{s}\left(\mathbb{R}^{2}\right)} .
$$

To prove (5) and (6), by Lemma 2.3, we first need to establish a desired estimate for $\tilde{\psi}_{u_{1} u_{2}} * \psi_{t_{1} t_{2}}$. By its definition, it is easy to show that, for all $\left(x_{1}, x_{2}\right) \in \mathbb{R}^{2}$,

$$
\tilde{\psi}_{u_{1} u_{2}} * \psi_{t_{1} t_{2}}\left(x_{1}, x_{2}\right)=\left(\tilde{\psi}_{u_{1}}^{(1)} * \psi_{t_{1}}^{(1)}\right) *_{2}\left(\tilde{\psi}_{u_{2}}^{(2)} * \psi_{t_{2}}^{(2)}\right)\left(x_{1}, x_{2}\right),
$$

where, and in what follows, $*_{2}$ denotes the convolution in the second variable. We also set $a \wedge b=\min \{a, b\}$ and $a \vee b=\max \{a, b\}$ for $a, b \in \mathbb{R}$. We claim that

(i) for all $t_{1}, u_{1}>0$ and all $\left(x_{1}, x_{2}\right) \in \mathbb{R}^{2}$,

$$
\left|\left(\tilde{\psi}_{u_{1}}^{(1)} * \psi_{t_{1}}^{(1)}\right)\left(x_{1}, x_{2}\right)\right| \leq C\left(\frac{u_{1}}{t_{1}} \wedge \frac{t_{1}}{u_{1}}\right) \frac{u_{1} \vee t_{1}}{\left(u_{1} \vee t_{1}+\left\|\left(x_{1}, x_{2}\right)\right\|\right)^{4}} ;
$$

(ii) for all $t_{2}, u_{2}>0$ and all $z \in \mathbb{R}$,

$$
\left|\left(\tilde{\psi}_{u_{2}}^{(2)} * \psi_{t_{2}}^{(2)}\right)(z)\right| \leq C\left(\frac{u_{2}}{t_{2}} \wedge \frac{t_{2}}{u_{2}}\right) \frac{u_{2} \vee t_{2}}{\left(u_{2} \vee t_{2}+|z|\right)^{2}} .
$$


By similarity, we only show (7). To this end, by the mean value theorem and some trivial computation, we can easily prove that, for all $u_{1}>0$ and $\left(x_{1}, x_{2}\right) \in \mathbb{R}^{2}$,

$$
\left|\tilde{\psi}_{u_{1}}^{(1)}\left(x_{1}, x_{2}\right)\right| \leq C \frac{u_{1}}{\left(u_{1}+\left\|\left(x_{1}, x_{2}\right)\right\|\right)^{4}},
$$

and, for all $u_{1}>0$ and $\left(x_{1}, x_{2}\right),\left(y_{1}, y_{2}\right) \in \mathbb{R}^{2}$ with $\left\|\left(y_{1}, y_{2}\right)\right\| \leq\left(u_{1}+\left\|\left(x_{1}, x_{2}\right)\right\|\right) / 2$,

$$
\left|\tilde{\psi}_{u_{1}}^{(1)}\left(x_{1}+y_{1}, x_{2}+y_{2}\right)-\tilde{\psi}_{u_{1}}^{(1)}\left(x_{1}, x_{2}\right)\right| \leq C \frac{\left\|\left(y_{1}, y_{2}\right)\right\|}{u_{1}+\left\|\left(x_{1}, x_{2}\right)\right\|} \frac{u_{1}}{\left(u_{1}+\left\|\left(x_{1}, x_{2}\right)\right\|\right)^{4}} .
$$

The estimates (9) and (10), with $\tilde{\psi}_{u_{1}}^{(1)}$ and $u_{1}$ replaced respectively by $\psi_{t_{1}}^{(1)}$ and $t_{1}$, also hold. We now prove $(7)$ in the case $u_{1} \geq t_{1}$. In this case, as

$$
\int_{\mathbb{R}^{2}} \psi_{t_{1}}^{(1)}\left(y_{1}, y_{2}\right) d y_{1} d y_{2}=0
$$

we can write

$$
\begin{aligned}
\left(\tilde{\psi}_{u_{1}}^{(1)} * \psi_{t_{1}}^{(1)}\right)\left(x_{1}, x_{2}\right) & \\
= & \int_{\left\|\left(y_{1}, y_{2}\right)\right\| \leq\left(u_{1}+\left\|\left(x_{1}, x_{2}\right)\right\|\right) / 2}\left[\tilde{\psi}_{u_{1}}^{(1)}\left(x_{1}-y_{1}, x_{2}-y_{2}\right)-\tilde{\psi}_{u_{1}}^{(1)}\left(x_{1}, x_{2}\right)\right] \\
& \quad \times \psi_{t_{1}}^{(1)}\left(y_{1}, y_{2}\right) d y_{1} d y_{2} \\
& +\int_{\left\|\left(y_{1}, y_{2}\right)\right\|>\left(u_{1}+\left\|\left(x_{1}, x_{2}\right)\right\|\right) / 2} \\
= & D_{1}+D_{2} .
\end{aligned}
$$

The estimate (10) yields that

$$
\begin{aligned}
\left|D_{1}\right| & \leq C \frac{t_{1}}{\left(u_{1}+\left\|\left(x_{1}, x_{2}\right)\right\|\right)^{4}} \int_{\mathbb{R}^{2}}\left\|\left(\frac{y_{1}}{t_{1}}, \frac{y_{2}}{t_{1}^{2}}\right)\right\|\left|\psi_{t_{1}}^{(1)}\left(y_{1}, y_{2}\right)\right| d y_{1} d y_{2} \\
& \leq C \frac{t_{1}}{\left(u_{1}+\left\|\left(x_{1}, x_{2}\right)\right\|\right)^{4}},
\end{aligned}
$$

and the estimates $(9)$ for $\tilde{\psi}_{u_{1}}^{(1)}$ and $\psi_{t_{1}}^{(1)}$ imply that

$$
\begin{aligned}
\left|D_{2}\right| \leq & C \int_{\left\|\left(y_{1}, y_{2}\right)\right\|>\left(u_{1}+\left\|\left(x_{1}, x_{2}\right)\right\|\right) / 2}\left|\tilde{\psi}_{u_{1}}^{(1)}\left(x_{1}-y_{1}, x_{2}-y_{2}\right)\right| \frac{t_{1}}{\left\|\left(y_{1}, y_{2}\right)\right\|^{4}} d y_{1} d y_{2} \\
& +C \frac{u_{1}}{\left(u_{1}+\left\|\left(x_{1}, x_{2}\right)\right\|\right)^{4}} \int_{\left\|\left(y_{1}, y_{2}\right)\right\|>u_{1} / 2} \frac{t_{1}}{\left\|\left(y_{1}, y_{2}\right)\right\|^{4}} d y_{1} d y_{2} \\
\leq & C \frac{t_{1}}{\left(u_{1}+\left\|\left(x_{1}, x_{2}\right)\right\|\right)^{4}},
\end{aligned}
$$


which proves (7).

Let $t, s>0$ and $\left(x_{1}, x_{2}\right) \in \mathbb{R}^{2}$. We now estimate

$$
\begin{aligned}
\int_{\mathbb{R}} \frac{t}{\left(t+\left\|\left(x_{1}, x_{2}\right)-(0, y)\right\|\right)^{4}} & \frac{s}{(s+|y|)^{2}} d y \\
\leq \int_{|y| \leq\left|x_{2}\right| / 2} & \frac{t}{\left(t^{2}+\left|x_{1}\right|^{2}+\left|x_{2}-y\right|\right)^{2}} \frac{s}{(s+|y|)^{2}} d y \\
& \quad+\int_{\left|x_{2}\right| / 2<|y| \leq 2\left|x_{2}\right|} \cdots+\int_{|y|>2\left|x_{2}\right|} \cdots=E_{1}+E_{2}+E_{3} .
\end{aligned}
$$

For $E_{1}$, in this case, we have that $\left|x_{2}-y\right| \geq\left|x_{2}\right| / 2$ and

$$
E_{1} \leq \frac{t}{\left(t^{2}+\left|x_{1}\right|^{2}+\left|x_{2}\right| / 2\right)^{2}} \int_{\mathbb{R}} \frac{s}{(s+|y|)^{2}} d y \leq C \frac{t}{\left(t+\left\|\left(x_{1}, x_{2}\right)\right\|\right)^{4}} ;
$$

for $E_{3}$, by the fact that

$$
t^{2}+\left|x_{1}\right|^{2}+\left|x_{2}-y\right| \geq t^{2}+\left|x_{1}\right|^{2}+\left|x_{2}\right| \geq\left(t+\left\|\left(x_{1}, x_{2}\right)\right\|\right)^{2} / 2,
$$

we also obtain an estimate similar to $E_{1}$. For $E_{2}$, we have

$$
E_{2} \leq C \frac{t}{t^{2}+\left|x_{1}\right|^{2}} \frac{s}{\left(s+\left|x_{2}\right|\right)^{2}} \int_{\mathbb{R}} \frac{1}{(1+|y|)^{2}} d y \leq C \frac{t}{\left(t+\left|x_{1}\right|\right)^{2}} \frac{s}{\left(s+\left|x_{2}\right|\right)^{2}} .
$$

Thus, for all $t, s>0$ and $\left(x_{1}, x_{2}\right) \in \mathbb{R}^{2}$,

$$
\begin{aligned}
\int_{\mathbb{R}} \frac{t}{\left(t+\left\|\left(x_{1}, x_{2}\right)-(0, y)\right\|\right)^{4}} \frac{s}{(s+|y|)^{2}} d y \\
\leq C\left\{\frac{t}{\left(t+\left\|\left(x_{1}, x_{2}\right)\right\|\right)^{4}}+\frac{t}{\left(t+\left|x_{1}\right|\right)^{2}} \frac{s}{\left(s+\left|x_{2}\right|\right)^{2}}\right\} .
\end{aligned}
$$

Let $M$ denote the Hardy-Littlewood maximal funnction on $\mathbb{R}^{2}$. Now, the estimates (7), (8), and (11) and Lemma 2.3 yield that

$$
\begin{array}{rl}
\mid \tilde{\psi}_{u_{1} u_{2}} * & f\left(x_{1}, x_{2}\right) \mid \\
\leq & C \int_{0}^{\infty} \int_{0}^{\infty}\left(\frac{u_{1}}{t_{1}} \wedge \frac{t_{1}}{u_{1}}\right)\left(\frac{u_{2}}{t_{2}} \wedge \frac{t_{2}}{u_{2}}\right) \\
& \times \int_{\mathbb{R}^{2}}\left\{\frac{u_{1} \vee t_{1}}{\left(u_{1} \vee t_{1}+\left\|\left(z_{1}, z_{2}\right)\right\|\right)^{4}}+\frac{u_{1} \vee t_{1}}{\left(u_{1} \vee t_{1}+\left|z_{1}\right|\right)^{2}} \frac{u_{2} \vee t_{2}}{\left(u_{2} \vee t_{2}+\left|z_{2}\right|\right)^{2}}\right\} \\
& \times\left|\psi_{t_{1} t_{2}} * f\left(x_{1}-z_{1}, x_{2}-z_{2}\right)\right| d z_{1} d z_{2} \frac{d t_{1}}{t_{1}} \frac{d t_{2}}{t_{2}} \\
\leq & C \int_{0}^{\infty} \int_{0}^{\infty}\left(\frac{u_{1}}{t_{1}} \wedge \frac{t_{1}}{u_{1}}\right)\left(\frac{u_{2}}{t_{2}} \wedge \frac{t_{2}}{u_{2}}\right) \\
& \times\left\{M\left(\psi_{t_{1} t_{2}} * f\right)\left(x_{1}, x_{2}\right)+M_{s}\left(\psi_{t_{1} t_{2}} * f\right)\left(x_{1}, x_{2}\right)\right\} \frac{d t_{1}}{t_{1}} \frac{d t_{2}}{t_{2}}
\end{array}
$$


which together with the Minkowski inequality and the $L^{p}\left(\mathbb{R}^{2}\right)$-boundedness of $M$ and $M_{s}$ yields that, for $p \in(1, \infty)$,

$$
\begin{aligned}
& \left\|\tilde{\psi}_{u_{1} u_{2}} * f\right\|_{L^{p}\left(\mathbb{R}^{2}\right)} \\
& \quad \leq C \int_{0}^{\infty} \int_{0}^{\infty}\left(\frac{u_{1}}{t_{1}} \wedge \frac{t_{1}}{u_{1}}\right)\left(\frac{u_{2}}{t_{2}} \wedge \frac{t_{2}}{u_{2}}\right)\left\|\psi_{t_{1} t_{2}} * f\right\|_{L^{p}\left(\mathbb{R}^{2}\right)} \frac{d t_{1}}{t_{1}} \frac{d t_{2}}{t_{2}} .
\end{aligned}
$$

The estimate (13) combined with the Minkowski inequality shows that

$$
\begin{aligned}
\|f\|_{0} \dot{B}_{p q}^{s}\left(\mathbb{R}^{2}\right) & \\
\leq & \left\{\int _ { 0 } ^ { \infty } \int _ { 0 } ^ { \infty } \left[\int_{0}^{u_{1}} \int_{0}^{u_{2}}\left(\frac{t_{1}}{u_{1}}\right)^{s_{1}+1}\left(\frac{t_{2}}{u_{2}}\right)^{s_{2}+1}\right.\right. \\
& \left.\left.\times t_{1}^{-s_{1}} t_{2}^{-s_{2}}\left\|\psi_{t_{1} t_{2}} * f\right\|_{L^{p}\left(\mathbb{R}^{2}\right)} \frac{d t_{1}}{t_{1}} \frac{d t_{2}}{t_{2}}\right]^{q} \frac{d u_{1}}{u_{1}} \frac{d u_{2}}{u_{2}}\right\}^{1 / q} \\
+ & C\left\{\int_{0}^{\infty} \int_{0}^{\infty}\left[\int_{0}^{u_{1}} \int_{u_{2}}^{\infty}\left(\frac{t_{1}}{u_{1}}\right)^{s_{1}+1}\left(\frac{t_{2}}{u_{2}}\right)^{s_{2}-1} \cdots \frac{d t_{1}}{t_{1}} \frac{d t_{2}}{t_{2}}\right]^{q} \frac{d u_{1}}{u_{1}} \frac{d u_{2}}{u_{2}}\right\}^{1 / q} \\
+ & C\left\{\int_{0}^{\infty} \int_{0}^{\infty}\left[\int_{u_{1}}^{\infty} \int_{0}^{u_{2}}\left(\frac{t_{1}}{u_{1}}\right)^{s_{1}-1}\left(\frac{t_{2}}{u_{2}}\right)^{s_{2}+1} \cdots \frac{d t_{1}}{t_{1}} \frac{d t_{2}}{t_{2}}\right]^{q} \frac{d u_{1}}{u_{1}} \frac{d u_{2}}{u_{2}}\right\}^{1 / q} \\
+ & C\left\{\int_{0}^{\infty} \int_{0}^{\infty}\left[\int_{u_{1}}^{\infty} \int_{u_{2}}^{\infty}\left(\frac{t_{1}}{u_{1}}\right)^{s_{1}-1}\left(\frac{t_{2}}{u_{2}}\right)^{s_{2}-1} \cdots \frac{d t_{1}}{t_{1}} \frac{d t_{2}}{t_{2}}\right]^{q} \frac{d u_{1}}{u_{1}} \frac{d u_{2}}{u_{2}}\right\}^{1 / q} \\
= & F_{1}+F_{2}+F_{3}+F_{4} .
\end{aligned}
$$

The Hölder inequality and the assumption that $s_{i}>-1$ for $i=1,2$ further imply that

$$
\begin{aligned}
F_{1} \leq & C\left\{\int_{0}^{\infty} \int_{0}^{\infty} t_{1}^{-s_{1} q} t_{2}^{-s_{2} q}\left\|\psi_{t_{1} t_{2}} * f\right\|_{L^{p}\left(\mathbb{R}^{2}\right)}^{q}\right. \\
\times & {\left.\left[\int_{t_{1}}^{\infty} \int_{t_{2}}^{\infty}\left(\frac{t_{1}}{u_{1}}\right)^{s_{1}+1}\left(\frac{t_{2}}{u_{2}}\right)^{s_{2}+1} \frac{d u_{1}}{u_{1}} \frac{d u_{2}}{u_{2}}\right] \frac{d t_{1}}{t_{1}} \frac{d t_{2}}{t_{2}}\right\}^{1 / q} } \\
\leq & C\left\{\int_{0}^{\infty} \int_{0}^{\infty} t_{1}^{-s_{1} q} t_{2}^{-s_{2} q}\left\|\psi_{t_{1} t_{2}} * f\right\|_{L^{p}\left(\mathbb{R}^{2}\right)}^{q} \frac{d t_{1}}{t_{1}} \frac{d t_{2}}{t_{2}}\right\}^{1 / q}=C\|f\|_{\dot{B}_{p q}^{s}\left(\mathbb{R}^{2}\right)},
\end{aligned}
$$

where, and in what follows, we denote by $q^{\prime}$ the conjugate index of $q$, namely, $1 / q+1 / q^{\prime}=1$.

The same argument as for $F_{1}$ also yields the desired estimates for $F_{i}$ with $i=2,3,4$. This proves (5) and hence, by symmetry, the independence of the norm $\|\cdot\|_{\dot{B}_{p q}^{s}\left(\mathbb{R}^{2}\right)}$ with respect to the choice of $\psi^{(i)}$, for $i=1,2$. 
We now turn to the proof of (6). From the estimate (12), it follows that, for all $u_{1}, u_{2}>0$ and $\left(x_{1}, x_{2}\right) \in \mathbb{R}^{2}$,

$$
\begin{aligned}
u_{1}^{-s_{1}} u_{2}^{-s_{2}}\left|\tilde{\psi}_{u_{1} u_{2}} * f\left(x_{1}, x_{2}\right)\right| & \\
\leq C\left\{\int_{0}^{\infty}\right. & \int_{0}^{\infty}\left(\frac{u_{1}}{t_{1}} \wedge \frac{t_{1}}{u_{1}}\right)\left(\frac{u_{2}}{t_{2}} \wedge \frac{t_{2}}{u_{2}}\right)\left(\frac{t_{1}}{u_{1}}\right)^{s_{1}}\left(\frac{t_{2}}{u_{2}}\right)^{s_{2}} \\
& \times\left[M\left(t_{1}^{-s_{1}} t_{2}^{-s_{2}} \psi_{t_{1} t_{2}} * f\right)\left(x_{1}, x_{2}\right)\right. \\
& \left.\left.+M_{s}\left(t_{1}^{-s_{1}} t_{2}^{-s_{2}} \psi_{t_{1} t_{2}} * f\right)\left(x_{1}, x_{2}\right)\right]^{q} \frac{d t_{1}}{t_{1}} \frac{d t_{2}}{t_{2}}\right\}^{1 / q},
\end{aligned}
$$

which combined with Lemma 2.3 and (10) yields that

$$
\begin{aligned}
\|f\|_{0} \dot{F}_{p q}^{s}\left(\mathbb{R}^{2}\right) \leq & C \|\left\{\int _ { 0 } ^ { \infty } \int _ { 0 } ^ { \infty } \left[M\left(t_{1}^{-s_{1}} t_{2}^{-s_{2}} \psi_{t_{1} t_{2}} * f\right)\right.\right. \\
& \left.\left.+M_{s}\left(t_{1}^{-s_{1}} t_{2}^{-s_{2}} \psi_{t_{1} t_{2}} * f\right)\right]^{q} \frac{d t_{1}}{t_{1}} \frac{d t_{2}}{t_{2}}\right\}^{1 / q} \|_{L^{p}\left(\mathbb{R}^{2}\right)} \\
\leq & C\left\|\left\{\int_{0}^{\infty} \int_{0}^{\infty} t_{1}^{-s_{1} q} t_{2}^{-s_{2} q}\left|\psi_{t_{1} t_{2}} * f\right|^{q} \frac{d t_{1}}{t_{1}} \frac{d t_{2}}{t_{2}}\right\}^{1 / q}\right\|_{L^{p}\left(\mathbb{R}^{2}\right)} \\
= & C\|f\|_{\dot{F}_{p q}^{s}\left(\mathbb{R}^{2}\right)},
\end{aligned}
$$

where we have used the vector-valued inequality of Fefferman-Stein in [1]. This proves (6) and, by symmetry, the independence of the norm $\|\cdot\|_{\dot{F}_{p q}^{s}\left(\mathbb{R}^{2}\right)}$ with respect to the choice of $\psi^{(i)}$ for $i=1,2$. This finishes the proof of Theorem 2.6.

Based on Theorem 2.6, we now introduce the Besov space $\dot{B}_{p q}^{s}\left(\mathbb{R}^{2}\right)$ and the TriebelLizorkin space $\dot{F}_{p q}^{s}\left(\mathbb{R}^{2}\right)$ as follows.

Definition 2.7. Let $s_{1}, s_{2} \in(-1,1)$ and $s=\left(s_{1}, s_{2}\right)$. The Besov space $\dot{B}_{p q}^{s}\left(\mathbb{R}^{2}\right)$ with $p, q \in[1, \infty]$ is defined by

$$
\dot{B}_{p q}^{s}\left(\mathbb{R}^{2}\right)=\left\{f \in \mathcal{S}_{\infty, F}\left(\mathbb{R}^{2}\right)^{\prime}:\|f\|_{\dot{B}_{p q}^{s}\left(\mathbb{R}^{2}\right)}<\infty\right\} ;
$$

and the Triebel-Lizorkin space $\dot{F}_{p q}^{s}\left(\mathbb{R}^{2}\right)$ with $p \in(1, \infty)$ and $q \in(1, \infty]$ is defined by

$$
\dot{F}_{p q}^{s}\left(\mathbb{R}^{2}\right)=\left\{f \in \mathcal{S}_{\infty, F}\left(\mathbb{R}^{2}\right)^{\prime}:\|f\|_{\dot{F}_{p q}^{s}\left(\mathbb{R}^{2}\right)}<\infty\right\} .
$$

Theorem 2.6 shows that the definitions of the spaces $\dot{B}_{p q}^{s}\left(\mathbb{R}^{2}\right)$ and $\dot{F}_{p q}^{s}\left(\mathbb{R}^{2}\right)$ are independent of the choices of $\psi^{(i)}$ with $i=1,2$.

From Lemma 2.3 and Lemma 2.4, we deduce the 'discrete' characterization of Besov spaces $\dot{B}_{p q}^{s}\left(\mathbb{R}^{2}\right)$ and Triebel-Lizorkin spaces $\dot{F}_{p q}^{s}\left(\mathbb{R}^{2}\right)$ as below. 
Theorem 2.8. Let $s_{1}, s_{2} \in(-1,1), s=\left(s_{1}, s_{2}\right)$ and all other notation be the same as in Lemma 2.4. Then $f \in \dot{B}_{p q}^{s}\left(\mathbb{R}^{2}\right)$ with $p, q \in[1, \infty]$ if and only if $f \in \mathcal{S}_{\infty, F}\left(\mathbb{R}^{2}\right)^{\prime}$ and

$$
\|f\|_{\dot{B}_{p q}^{s}\left(\mathbb{R}^{2}\right)}^{\prime}=\left\{\sum_{k_{1}=-\infty}^{\infty} \sum_{k_{2}=-\infty}^{\infty} 2^{k_{1} s_{1} q} 2^{k_{2} s_{2} q}\left\|\psi_{k_{1} k_{2}} * f\right\|_{L^{p}\left(\mathbb{R}^{2}\right)}^{q}\right\}^{1 / q}<\infty ;
$$

and $f \in \dot{F}_{p q}^{s}\left(\mathbb{R}^{2}\right)$ with $p \in(1, \infty)$ and $q \in(1, \infty]$ if and only if $f \in \mathcal{S}_{\infty, F}\left(\mathbb{R}^{2}\right)^{\prime}$ and

$$
\|f\|_{\dot{F}_{p q}^{s}\left(\mathbb{R}^{2}\right)}^{\prime}=\left\|\left\{\sum_{k_{1}=-\infty}^{\infty} \sum_{k_{2}=-\infty}^{\infty} 2^{k_{1} s_{1} q} 2^{k_{2} s_{2} q}\left|\psi_{k_{1} k_{2}} * f\right|^{q}\right\}^{1 / q}\right\|_{L^{p}\left(\mathbb{R}^{2}\right)}<\infty .
$$

Furthermore, in this case, $\|\cdot\|_{\dot{B}_{p q}^{s}\left(\mathbb{R}^{2}\right)}^{\prime}$ and $\|\cdot\|_{\dot{F}_{p q}^{s}\left(\mathbb{R}^{2}\right)}^{\prime}$ are equivalent to $\|\cdot\|_{\dot{B}_{p q}^{s}\left(\mathbb{R}^{2}\right)}$ and $\|\cdot\|_{\dot{F}_{p q}^{s}\left(\mathbb{R}^{2}\right)}$, respectively.

Since the proof of Theorem 2.8 is essentially the same as that of Theorem 2.6, we omit the details.

The following properties of these spaces can easily be deduced from Theorem 2.8 and the monotonicity of the spaces $l^{q}$; see $[9,11]$.

Proposition 2.9. Let $s_{1}, s_{2} \in(-1,1)$ and $s=\left(s_{1}, s_{2}\right)$. Then

(i) If $p \in[1, \infty]$ and $1 \leq q_{1} \leq q_{2} \leq \infty$, then $\dot{B}_{p q_{1}}^{s}\left(\mathbb{R}^{2}\right) \subset \dot{B}_{p q_{2}}^{s}\left(\mathbb{R}^{2}\right)$, namely, there exists a positive constant $C$ such that, for all $f \in \dot{B}_{p q_{1}}^{s}\left(\mathbb{R}^{2}\right)$,

$$
\|f\|_{\dot{B}_{p q_{2}}^{s}\left(\mathbb{R}^{2}\right)} \leq C\|f\|_{\dot{B}_{p q_{1}}^{s}\left(\mathbb{R}^{2}\right)} .
$$

(ii) If $p \in(1, \infty)$ and $1<q_{1} \leq q_{2} \leq \infty$, then $\dot{F}_{p q_{1}}^{s}\left(\mathbb{R}^{2}\right) \subset \dot{F}_{p q_{2}}^{s}\left(\mathbb{R}^{2}\right)$, namely, there exists a positive constant $C$ such that, for all $f \in \dot{F}_{p q_{1}}^{s}\left(\mathbb{R}^{2}\right)$,

$$
\|f\|_{\dot{F}_{p q_{2}}^{s}\left(\mathbb{R}^{2}\right)} \leq C\|f\|_{\dot{F}_{p q_{1}}^{s}\left(\mathbb{R}^{2}\right)} .
$$

(iii) If $p \in(1, \infty)$ and $q \in(1, \infty]$, then

$$
\dot{B}_{p, \min (p, q)}^{s}\left(\mathbb{R}^{2}\right) \subset \dot{F}_{p q}^{s}\left(\mathbb{R}^{2}\right) \subset \dot{B}_{p, \max (p, q)}^{s}\left(\mathbb{R}^{2}\right),
$$

namely, there exists a positive constant $C$ such that, for all $f \in \dot{B}_{p, \min (p, q)}^{s}\left(\mathbb{R}^{2}\right)$,

$$
\|f\|_{\dot{B}_{p, \max (p, q)}^{s}\left(\mathbb{R}^{2}\right)} \leq C\|f\|_{\dot{F}_{p q}^{s}\left(\mathbb{R}^{2}\right)} \leq C\|f\|_{\dot{B}_{p, \min (p, q)}^{s}\left(\mathbb{R}^{2}\right)} .
$$

The following basic properties are useful in the study on the dual and interpolation of these function spaces; see $[4,9]$. 
Proposition 2.10. Let $s_{1}, s_{2} \in(-1,1)$ and $s=\left(s_{1}, s_{2}\right)$. Then

(i) The space $\dot{B}_{p q}^{s}\left(\mathbb{R}^{2}\right)$ is a Banach space and $\mathcal{S}_{\infty, F}\left(\mathbb{R}^{2}\right) \subset \dot{B}_{p q}^{s}\left(\mathbb{R}^{2}\right) \subset \mathcal{S}_{\infty, F}\left(\mathbb{R}^{2}\right)^{\prime}$ for $p, q \in[1, \infty]$. If $p, q \in[1, \infty)$, then $\mathcal{S}_{\infty, F}\left(\mathbb{R}^{2}\right)$ is dense in $\dot{B}_{p q}^{s}\left(\mathbb{R}^{2}\right)$.

(ii) The space $\dot{F}_{p q}^{s}\left(\mathbb{R}^{2}\right)$ is a Banach space and $\mathcal{S}_{\infty, F}\left(\mathbb{R}^{2}\right) \subset \dot{F}_{p q}^{s}\left(\mathbb{R}^{2}\right) \subset \mathcal{S}_{\infty, F}\left(\mathbb{R}^{2}\right)^{\prime}$ for $p \in(1, \infty)$ and $q \in(1, \infty]$. If $p, q \in(1, \infty)$, then $\mathcal{S}_{\infty, F}\left(\mathbb{R}^{2}\right)$ is dense in $\dot{F}_{p q}^{s}\left(\mathbb{R}^{2}\right)$.

Proof. We only prove the conclusion that $\mathcal{S}_{\infty, F}\left(\mathbb{R}^{2}\right) \subset \dot{B}_{p q}^{s}\left(\mathbb{R}^{2}\right)$ of (i), and the conclusion that $\dot{B}_{p q}^{s}\left(\mathbb{R}^{2}\right) \subset \mathcal{S}_{\infty, F}\left(\mathbb{R}^{2}\right)^{\prime}$ can be deduced from Lemma 2.3 and the Hölder inequality. Moreover, by a routine procedure, we can prove that $\mathcal{S}_{\infty}\left(\mathbb{R}^{2}\right)$ is dense in $\dot{B}_{p q}^{s}\left(\mathbb{R}^{2}\right)$ for $p, q \in[1, \infty)$ and we omit the details; see [4].

Let $f \in \mathcal{S}_{\infty, F}\left(\mathbb{R}^{2}\right)$. By Definition 2.1, there exists a Schwartz function $f^{\sharp} \in \mathcal{S}\left(\mathbb{R}^{2} \times \mathbb{R}\right)$ satisfying all the properties of Definition 2.1 such that, for all $\left(x_{1}, x_{2}\right) \in \mathbb{R}^{2}$,

$$
f\left(x_{1}, x_{2}\right)=\int_{\mathbb{R}} f^{\sharp}\left(x_{1}, x_{2}-x_{3}, x_{3}\right) d x_{3} .
$$

Let $\psi_{t_{1} t_{2}}$ be the same as in Lemma 2.3, and we write

$$
\begin{aligned}
\|f\|_{\dot{B}_{p q}^{s}\left(\mathbb{R}^{2}\right)} \leq & \left\{\int_{0}^{1} \int_{0}^{1} t_{1}^{-s_{1} q} t_{2}^{-s_{2} q}\left\|\psi_{t_{1} t_{2}} * f\right\|_{L^{p}\left(\mathbb{R}^{2}\right)}^{q} \frac{d t_{1}}{t_{1}} \frac{d t_{2}}{t_{2}}\right\}^{1 / q} \\
& +\left\{\int_{0}^{1} \int_{1}^{\infty} \cdots \frac{d t_{1}}{t_{1}} \frac{d t_{2}}{t_{2}}\right\}^{1 / q}+\left\{\int_{1}^{\infty} \int_{0}^{1} \cdots \frac{d t_{1}}{t_{1}} \frac{d t_{2}}{t_{2}}\right\}^{1 / q} \\
& +\left\{\int_{1}^{\infty} \int_{1}^{\infty} \cdots \frac{d t_{1}}{t_{1}} \frac{d t_{2}}{t_{2}}\right\}^{1 / q} \\
= & L_{1}+L_{2}+L_{3}+L_{4} .
\end{aligned}
$$

To estimate $L_{1}$, for $\left(x_{1}, x_{2}\right) \in \mathbb{R}^{2}$ and $t_{1}, t_{2}>0$, by the vanishing moments of $\psi^{(1)}$ and $\psi^{(2)}$, we write

$$
\begin{aligned}
\psi_{t_{1} t_{2}} * f\left(x_{1}, x_{2}\right) & \\
=\int_{\mathbb{R}} & \int_{\left|z-y_{3}\right| \leq|z| / 2} \int_{\left\|\left(x_{1}-y_{1}, x_{2}-y_{2}-z\right)\right\| \leq\left\|\left(x_{1}, x_{2}-z\right)\right\| / 2} \psi_{t_{1}}^{(1)}\left(x_{1}-y_{1}, x_{2}-y_{2}-z\right) \\
\quad \times & \psi_{t_{2}}^{(2)}\left(z-y_{3}\right)\left\{\left[f^{\sharp}\left(y_{1}, y_{2}, y_{3}\right)-f^{\sharp}\left(x_{1}, x_{2}-z, y_{3}\right)\right]\right. \\
& \left.-\left[f^{\sharp}\left(y_{1}, y_{2}, z\right)-f^{\sharp}\left(x_{1}, x_{2}-z, z\right)\right]\right\} d y_{1} d y_{2} d y_{3} d z+
\end{aligned}
$$




$$
\begin{aligned}
& +\int_{\mathbb{R}} \int_{\left|z-y_{3}\right|>|z| / 2} \int_{\left\|\left(x_{1}-y_{1}, x_{2}-y_{2}-z\right)\right\| \leq\left\|\left(x_{1}, x_{2}-z\right)\right\| / 2} \cdots \\
& +\int_{\mathbb{R}} \int_{\left|z-y_{3}\right| \leq|z| / 2} \int_{\left\|\left(x_{1}-y_{1}, x_{2}-y_{2}-z\right)\right\|>\left\|\left(x_{1}, x_{2}-z\right)\right\| / 2} \cdots \\
& +\int_{\mathbb{R}} \int_{\left|z-y_{3}\right|>|z| / 2} \int_{\left\|\left(x_{1}-y_{1}, x_{2}-y_{2}-z\right)\right\|>\left\|\left(x_{1}, x_{2}-z\right)\right\| / 2}^{\cdots}
\end{aligned}
$$

Then, the same argument as that for $I_{i}$ with $i=1,2,3,4$ in the proof of Lemma 2.3 yields that, for any $N \in \mathbb{Z}$, there exists a positive constant $C=C_{N, f}$ such that, for all $t_{1}, t_{2}>0$ and $\left(x_{1}, x_{2}\right) \in \mathbb{R}^{2}$,

$$
\left|\psi_{t_{1} t_{2}} * f\left(x_{1}, x_{2}\right)\right| \leq C t_{1} t_{2} \frac{1}{\left(1+\left\|\left(x_{1}, x_{2}\right)\right\|\right)^{N}},
$$

which implies the desired estimate for $L_{1}$. The same argument as that for $J_{i}$ with $i=1,2,3,4$ in the proof of Lemma 2.3 can yield a desired estimate for $L_{2}$. The estimates for $L_{3}$ and $L_{4}$ can be obtained in a similar way. We omit the details. This finishes the proof of Proposition 2.9.

Based on Proposition 2.10, we can give out the dual spaces of these spaces as below, which can be proved by an argument same as that of Theorem 7.1 in [4]. We omit the details.

Proposition 2.11. Let $s_{1}, s_{2} \in(-1,1), s=\left(s_{1}, s_{2}\right)$, and $-s=\left(-s_{1},-s_{2}\right)$. Then

(i) $\left(\dot{B}_{p q}^{s}\left(\mathbb{R}^{2}\right)\right)^{*}=\dot{B}_{p^{\prime} q^{\prime}}^{-s}\left(\mathbb{R}^{2}\right)$ for $p, q \in[1, \infty)$ with $1 / p+1 / p^{\prime}=1 / q+1 / q^{\prime}=1$. More precisely, given $g \in \dot{B}_{p^{\prime} q^{\prime}}^{-s}\left(\mathbb{R}^{2}\right)$, then $\mathcal{L}_{g}(f)=\langle f, g\rangle$ defines a linear functional on $\mathcal{S}_{\infty, F}\left(\mathbb{R}^{2}\right)$ such that

$$
\left|\mathcal{L}_{g}(f)\right| \leq C\|f\|_{\dot{B}_{p q}^{s}\left(\mathbb{R}^{2}\right)}\|g\|_{\dot{B}_{p^{\prime} q^{\prime}}^{-s}\left(\mathbb{R}^{2}\right)},
$$

and this linear functional can be extended to $\dot{B}_{p q}^{s}\left(\mathbb{R}^{2}\right)$ with norm at most $C\|g\|_{\dot{B}_{p^{\prime} q^{\prime}}^{-s}\left(\mathbb{R}^{2}\right)}$.

Conversely, if $\mathcal{L}$ is a linear functional on $\dot{B}_{p q}^{s}\left(\mathbb{R}^{2}\right)$, then there exists a unique $g \in \dot{B}_{p^{\prime} q^{\prime}}^{-s}\left(\mathbb{R}^{2}\right)$ such that $\mathcal{L}_{g}(f)=\langle f, g\rangle$ defines a linear functional on $\mathcal{S}_{\infty, F}\left(\mathbb{R}^{2}\right)$, and $\mathcal{L}$ is the extension of $\mathcal{L}_{g}$ with $\|g\|_{\dot{B}_{p^{\prime} q^{\prime}}^{-s}\left(\mathbb{R}^{2}\right)} \leq C\|\mathcal{L}\|$.

(ii) $\left(\dot{F}_{p q}^{s}\left(\mathbb{R}^{2}\right)\right)^{*}=F_{p^{\prime} q^{\prime}}^{-s}\left(\mathbb{R}^{2}\right)$ for $p, q \in(1, \infty)$ with $1 / p+1 / p^{\prime}=1 / q+1 / q^{\prime}=1$. More precisely, given $g \in \dot{F}_{p^{\prime} q^{\prime}}^{-s}\left(\mathbb{R}^{2}\right)$, then $\mathcal{L}_{g}(f)=\langle f, g\rangle$ defines a linear functional on $\mathcal{S}_{\infty, F}\left(\mathbb{R}^{2}\right)$ such that

$$
\left|\mathcal{L}_{g}(f)\right| \leq C\|f\|_{\dot{F}_{p q}^{s}\left(\mathbb{R}^{2}\right)}\|g\|_{\dot{F}_{p^{\prime} q^{\prime}}^{-s}\left(\mathbb{R}^{2}\right)},
$$


and this linear functional can be extended to $\dot{F}_{p q}^{s}\left(\mathbb{R}^{2}\right)$ with norm at most $C\|g\|_{\dot{F}_{p^{\prime} q^{\prime}}^{-s}\left(\mathbb{R}^{2}\right)}$.

Conversely, if $\mathcal{L}$ is a linear functional on $\dot{F}_{p q}^{s}\left(\mathbb{R}^{2}\right)$, then there exists a unique $g \in \dot{F}_{p^{\prime} q^{\prime}}^{-s}\left(\mathbb{R}^{2}\right)$ such that $\mathcal{L}_{g}(f)=\langle f, g\rangle$ defines a linear functional on $\mathcal{S}_{\infty, F}\left(\mathbb{R}^{2}\right)$, and $\mathcal{L}$ is the extension of $\mathcal{L}_{g}$ with $\|g\|_{\dot{F}_{p^{\prime} q^{\prime}}^{-s}\left(\mathbb{R}^{2}\right)} \leq C\|\mathcal{L}\|$.

Remark 2.12. For $s_{1}, s_{2} \in(-1,1), s=\left(s_{1}, s_{2}\right)$ and $p, q \in[1, \infty]$, let us now define ${ }_{\circ} \dot{B}_{p q}^{s}\left(\mathbb{R}^{2}\right)$ to be the completion of $\mathcal{S}_{\infty, F}\left(\mathbb{R}^{2}\right)$ in $\dot{B}_{p q}^{s}\left(\mathbb{R}^{2}\right)$ endowed with the same norm as $\dot{B}_{p q}^{s}\left(\mathbb{R}^{2}\right)$. Then, in the sense of Proposition 2.11, we have

$$
\left({ }_{\circ} \dot{B}_{p q}^{s}\left(\mathbb{R}^{2}\right)\right)^{*}=B_{p^{\prime} q^{\prime}}^{-s}\left(\mathbb{R}^{2}\right)
$$

with $-s, p^{\prime}$, and $q^{\prime}$ having the same meaning as in Proposition 2.11. The equality (14) is new only for the case $\max (p, q)=\infty$ in comparison with Proposition 2.11. This fact can be easily proved by combining the argument in [4, pp. 116-120] with that in [9, p. 180]; see also [10, pp. 121, 122]. We omit the details.

Now, using these properties, we establish the Lusin-area characterizations of Triebel-Lizorkin spaces $\dot{F}_{p q}^{s}\left(\mathbb{R}^{2}\right)$. First we introduce the following two kinds of Lusinarea functions.

Definition 2.13. Let $s_{i} \in \mathbb{R}$ and $\alpha_{i}>0$ for $i=1,2, s=\left(s_{1}, s_{2}\right)$ and $q \in(1, \infty)$. Let $\psi_{t_{1} t_{2}}$ for $t_{i}>0$ with $i=1,2$ be the same as in Lemma 2.3 and $\chi=\chi_{(0,1)}$. For $f \in \mathcal{S}_{\infty, F}\left(\mathbb{R}^{2}\right)^{\prime}$ and $\left(x_{1}, x_{2}\right) \in \mathbb{R}^{2}$, we define

$$
\begin{aligned}
S_{q ; \alpha_{1}, \alpha_{2}}^{s}(f)\left(x_{1}, x_{2}\right)= & \left\{\int_{0}^{\infty} \int_{0}^{\infty} \int_{\mathbb{R}^{2}} \chi\left(\frac{\left|x_{1}-y_{1}\right|}{\alpha_{1} t_{1}}\right) \chi\left(\frac{\left|x_{2}-y_{2}\right|}{\alpha_{2} t_{2}}\right)\right. \\
& \left.\times t_{1}^{-s_{1} q} t_{2}^{-s_{2} q}\left|\psi_{t_{1} t_{2}} * f\left(y_{1}, y_{2}\right)\right|^{q} d y_{1} d y_{2} \frac{d t_{1}}{t_{1}^{2}} \frac{d t_{2}}{t_{2}^{2}}\right\}^{1 / q}
\end{aligned}
$$

and

$$
\begin{aligned}
\tilde{S}_{q ; \alpha_{1}, \alpha_{2}}^{s}(f)\left(x_{1}, x_{2}\right)= & \left\{\int_{0}^{\infty} \int_{0}^{t_{1}^{2}} \int_{\mathbb{R}^{2}} \chi\left(\frac{\left|x_{1}-y_{1}\right|}{\alpha_{1} t_{1}}\right) \chi\left(\frac{\left|x_{2}-y_{2}\right|}{\alpha_{2} t_{1}^{2}}\right)\right. \\
& \left.\times t_{1}^{-s_{1} q} t_{2}^{-s_{2} q}\left|\psi_{t_{1} t_{2}} * f\left(y_{1}, y_{2}\right)\right|^{q} d y_{1} d y_{2} \frac{d t_{2}}{t_{1}^{2} t_{2}} \frac{d t_{1}}{t_{1}^{2}}\right\}^{1 / q} \\
& +\left\{\int_{0}^{\infty} \int_{t_{1}^{2}}^{\infty} \int_{\mathbb{R}^{2}} \chi\left(\frac{\left|x_{1}-y_{1}\right|}{\alpha_{1} t_{1}}\right) \chi\left(\frac{\left|x_{2}-y_{2}\right|}{\alpha_{2} t_{2}}\right)\right. \\
& \left.\times t_{1}^{-s_{1} q} t_{2}^{-s_{2} q}\left|\psi_{t_{1} t_{2}} * f\left(y_{1}, y_{2}\right)\right|^{q} d y_{1} d y_{2} \frac{d t_{2}}{t_{2}^{2}} \frac{d t_{1}}{t_{1}^{2}}\right\}^{1 / q} .
\end{aligned}
$$

The characterizations of Lusin-area functions of $\dot{F}_{p q}^{s}\left(\mathbb{R}^{2}\right)$ can be stated as below. 
Theorem 2.14. Let $s_{1}, s_{2} \in(-1,1), s=\left(s_{1}, s_{2}\right), \alpha_{i}>0$ for $i=1,2$, and $p, q \in$ $(1, \infty)$. Then, for $f \in \mathcal{S}_{\infty, F}\left(\mathbb{R}^{2}\right)^{\prime}$, the following three propositions are equivalent:

(i) $f \in \dot{F}_{p q}^{s}\left(\mathbb{R}^{2}\right)$;

(ii) $S_{q ; \alpha_{1}, \alpha_{2}}^{s}(f) \in L^{p}\left(\mathbb{R}^{2}\right)$;

(iii) $\tilde{S}_{q ; \alpha_{1}, \alpha_{2}}^{s}(f) \in L^{p}\left(\mathbb{R}^{2}\right)$.

Furthermore, in this case,

$$
\|f\|_{\dot{F}_{p q}^{s}\left(\mathbb{R}^{2}\right)} \sim\left\|S_{q ; \alpha_{1}, \alpha_{2}}^{s}(f)\right\|_{L^{p}\left(\mathbb{R}^{2}\right)} \sim\left\|\tilde{S}_{q ; \alpha_{1}, \alpha_{2}}^{s}(f)\right\|_{L^{p}\left(\mathbb{R}^{2}\right)} .
$$

Proof. Letting $f \in \dot{F}_{p q}^{s}\left(\mathbb{R}^{2}\right)$, we first prove that $S_{q ; \alpha_{1}, \alpha_{2}}^{s}(f), \tilde{S}_{q ; \alpha_{1}, \alpha_{2}}^{s}(f) \in L^{p}\left(\mathbb{R}^{2}\right)$ and there exists a positive constant $C$ such that, for all $f \in \dot{F}_{p q}^{s}\left(\mathbb{R}^{2}\right)$,

$$
\left\|S_{q ; \alpha_{1}, \alpha_{2}}^{s}(f)\right\|_{L^{p}\left(\mathbb{R}^{2}\right)} \leq C\|f\|_{\dot{F}_{p q}^{s}\left(\mathbb{R}^{2}\right)}
$$

and

$$
\left\|\tilde{S}_{q ; \alpha_{1}, \alpha_{2}}^{s}(f)\right\|_{L^{p}\left(\mathbb{R}^{2}\right)} \leq C\|f\|_{\dot{F}_{p q}^{s}\left(\mathbb{R}^{2}\right)} .
$$

To this end, for $\left(z_{1}, z_{2}\right) \in \mathbb{R}^{2}, t_{i}>0,\left|x_{i}-y_{i}\right|<\alpha_{i} u_{i}$ with $u_{i}>0, i=1,2$, by (11), we have

$$
\begin{aligned}
\int_{\mathbb{R}} \frac{u_{1} \vee t_{1}}{\left(u_{1} \vee t_{1}+\left\|\left(y_{1}-z_{1}, y_{2}-z_{2}\right)-(0, w)\right\|\right)^{4}} \cdot \frac{u_{2} \vee t_{2}}{\left(u_{2} \vee t_{2}+|w|\right)^{2}} d w \\
\leq C\left\{\frac{u_{1} \vee t_{1}}{\left(u_{1} \vee t_{1}+\left\|\left(x_{1}-z_{1}, x_{2}-z_{2}\right)\right\|\right)^{4}}\right. \\
\left.\quad+\frac{u_{1} \vee t_{1}}{\left(u_{1} \vee t_{1}+\left|x_{1}-z_{1}\right|\right)^{2}} \frac{u_{2} \vee t_{2}}{\left(u_{2} \vee t_{2}+\left|x_{2}-z_{2}\right|\right)^{2}}\right\}
\end{aligned}
$$

and, similarly, for $\left|x_{1}-y_{1}\right|<\alpha_{1} u_{1}$ and $\left|x_{2}-y_{2}\right|<\alpha_{2} u_{1}^{2}$, we also have

$$
\begin{aligned}
\int_{\mathbb{R}} \frac{u_{1} \vee t_{1}}{\left(u_{1} \vee t_{1}+\left\|\left(y_{1}-z_{1}, y_{2}-z_{2}\right)-(0, w)\right\|\right)^{4}} \cdot \frac{u_{2} \vee t_{2}}{\left(u_{2} \vee t_{2}+|w|\right)^{2}} d w \\
\leq C\left\{\frac{u_{1} \vee t_{1}}{\left(u_{1} \vee t_{1}+\left\|\left(x_{1}-z_{1}, x_{2}-z_{2}\right)\right\|\right)^{4}}\right. \\
\left.\quad+\frac{u_{1} \vee t_{1}}{\left(u_{1} \vee t_{1}+\left|x_{1}-z_{1}\right|\right)^{2}} \frac{u_{2} \vee t_{2}}{\left(u_{2} \vee t_{2}+\left|x_{2}-z_{2}\right|\right)^{2}}\right\}
\end{aligned}
$$

Now the estimates (7), (8), Lemma 2.3, and the estimates (17) and (18), respectively, 
yield that

$$
\begin{aligned}
& \chi\left(\frac{\left|x_{1}-y_{1}\right|}{\alpha_{1} u_{1}}\right) \chi\left(\frac{\left|x_{2}-y_{2}\right|}{\alpha_{2} u_{2}}\right)\left|\psi_{u_{1} u_{2}} * f\left(y_{1}, y_{2}\right)\right| \\
& \leq C \int_{0}^{\infty} \int_{0}^{\infty}\left(\frac{u_{1}}{t_{1}} \wedge \frac{t_{1}}{u_{1}}\right)\left(\frac{u_{2}}{t_{2}} \wedge \frac{t_{2}}{u_{2}}\right) \\
& \times\left\{M\left(\psi_{t_{1} t_{2}} * f\right)\left(x_{1}, x_{2}\right)+M_{s}\left(\psi_{t_{1} t_{2}} * f\right)\left(x_{1}, x_{2}\right)\right\} \frac{d t_{1}}{t_{1}} \frac{d t_{2}}{t_{2}}
\end{aligned}
$$

and

$$
\begin{aligned}
\chi\left(\frac{\left|x_{1}-y_{1}\right|}{\alpha_{1} u_{1}}\right) \chi & \chi\left(\frac{\left|x_{2}-y_{2}\right|}{\alpha_{2} u_{1}^{2}}\right)\left|\psi_{u_{1} u_{2}} * f\left(y_{1}, y_{2}\right)\right| \\
\leq & C \int_{0}^{\infty} \int_{0}^{\infty}\left(\frac{u_{1}}{t_{1}} \wedge \frac{t_{1}}{u_{1}}\right)\left(\frac{u_{2}}{t_{2}} \wedge \frac{t_{2}}{u_{2}}\right) \\
& \times\left\{M\left(\psi_{t_{1} t_{2}} * f\right)\left(x_{1}, x_{2}\right)+M_{s}\left(\psi_{t_{1} t_{2}} * f\right)\left(x_{1}, x_{2}\right)\right\} \frac{d t_{1}}{t_{1}} \frac{d t_{2}}{t_{2}} .
\end{aligned}
$$

Replacing (12) with (19) and (20), respectively, and repeating the argument of (6) in Theorem 2.6, we then obtain (15) and (16).

We now show the converse of (15) and (16) and by similarity we only prove the converse of (16). To this end, letting $f \in \dot{F}_{p q}^{s}\left(\mathbb{R}^{2}\right)$ and $g \in \dot{F}_{p^{\prime} q^{\prime}}^{-s}\left(\mathbb{R}^{2}\right)$ and $\left\{\psi_{t_{1} t_{2}}\right\}_{t_{1}, t_{2}>0}$ be the same as in Lemma 2.3, by (16), Proposition 2.11, and the Hölder inequality, we obtain

$$
\begin{aligned}
\|f\|_{\dot{F}_{p q}^{s}\left(\mathbb{R}^{2}\right)} & =\sup _{\|g\|_{\dot{F}_{p^{\prime} q^{\prime}}^{-s}\left(\mathbb{R}^{2}\right)} \leq 1}\left|\int_{0}^{\infty} \int_{0}^{\infty}\left\langle\psi_{t_{1} t_{2}} * f, \psi_{t_{1} t_{2}} * g\right\rangle \frac{d t_{1}}{t_{1}} \frac{d t_{2}}{t_{2}}\right| \\
& \leq C \sup _{\|g\|_{\dot{F}_{p^{\prime} q^{\prime}}^{-s}}^{-s}\left(\mathbb{R}^{2}\right)} \leq 1 \\
& \leq C\left\|\tilde{S}_{q ; \alpha_{1}, \alpha_{2}}^{s}(f)\right\|_{\mathbb{R}^{p}\left(\mathbb{R}^{2}\right)} \tilde{S}_{q ; \alpha_{1}, \alpha_{2}}^{s}(f)\left(x_{1}, x_{2}\right) \tilde{S}_{q^{\prime} ; \alpha_{1}, \alpha_{2}}^{-s}(g)\left(x_{1}, x_{2}\right) d x_{1} d x_{2}
\end{aligned}
$$

which establishes the equivalence of (i) and (ii) and, hence, completes the proof of Theorem 2.14.

Remark 2.15. From the proof of Theorem 2.14, it is easy to see that (15) and (16) also hold for $p \in(1, \infty)$ and $q=\infty$.

Let us now state the Littlewood-Paley theorem corresponding to the following $g$-function.

Definition 2.16. With the same notation as in Lemma 2.3, for $f \in \mathcal{S}_{\infty, F}\left(\mathbb{R}^{2}\right)^{\prime}$, we define its Littlewood-Paley $g$-function, $g(f)$, of $f$ by

$$
g(f)\left(x_{1}, x_{2}\right)=\left\{\int_{0}^{\infty} \int_{0}^{\infty}\left|\psi_{t_{1} t_{2}} * f\left(x_{1}, x_{2}\right)\right|^{2} \frac{d t_{1}}{t_{1}} \frac{d t_{2}}{t_{2}}\right\}^{1 / 2},
$$


where $\left(x_{1}, x_{2}\right) \in \mathbb{R}^{2}$.

Using the boundedness of vector-valued singular integrals and a dual argument similar to the proof of Theorem 2.14, we can obtain the following result and we omit the details; see [3].

Theorem 2.17. Let $f \in \mathcal{S}_{\infty, F}\left(\mathbb{R}^{2}\right)^{\prime}$ and $p \in(1, \infty)$. Then the norm $\|g(f)\|_{L^{p}\left(\mathbb{R}^{2}\right)}$ is equivalent to the norm $\|f\|_{L^{p}\left(\mathbb{R}^{2}\right)}$.

From Theorem 2.17, Definition 2.7, and Theorem 2.14, it is easy to deduce the following conclusions:

Corollary 2.18. Let $p \in(1, \infty)$. Then $\dot{F}_{p 2}^{0}\left(\mathbb{R}^{2}\right)=L^{p}\left(\mathbb{R}^{2}\right)$ with an equivalent norm. Moreover, the norms $\|f\|_{L^{p}\left(\mathbb{R}^{2}\right)},\|g(f)\|_{L^{p}\left(\mathbb{R}^{2}\right)},\left\|S_{2 ; \alpha_{1}, \alpha_{2}}^{(0,0)}\right\|_{L^{p}\left(\mathbb{R}^{2}\right)}$, and $\left\|\tilde{S}_{2 ; \alpha_{1}, \alpha_{2}}^{(0,0)}\right\|_{L^{p}\left(\mathbb{R}^{2}\right)}$ are mutually equivalent, where $\alpha_{1}, \alpha_{2}>0$.

We now establish a new characterization of Besov and Triebel-Lizorkin spaces. First, we introduce the following new Besov and Triebel-Lizorkin norms.

Definition 2.19. Let $\psi_{t_{1} t_{2}}$ be the same as in Lemma 2.1 and $s_{1}, s_{2} \in \mathbb{R}$. For $f \in \mathcal{S}_{\infty, F}\left(\mathbb{R}^{2}\right)^{\prime}$, define

$$
\|f\|_{1 \dot{B}_{p q}^{s}\left(\mathbb{R}^{2}\right)}=\left\{\int_{0}^{\infty} \int_{0}^{\infty} t_{1}^{-s_{1} q}\left(t_{1}^{2}+t_{2}\right)^{-s_{2} q}\left\|\psi_{t_{1} t_{2}} * f\right\|_{L^{p}\left(\mathbb{R}^{2}\right)}^{q} \frac{d t_{1}}{t_{1}} \frac{d t_{2}}{t_{2}}\right\}^{1 / q}
$$

for $p, q \in[1, \infty]$, and

$$
\|f\|_{1 \dot{F}_{p q}^{s}\left(\mathbb{R}^{2}\right)}=\left\|\left\{\int_{0}^{\infty} \int_{0}^{\infty} t_{1}^{-s_{1} q}\left(t_{1}^{2}+t_{2}\right)^{-s_{2} q}\left|\psi_{t_{1} t_{2}} * f\right|^{q} \frac{d t_{1}}{t_{1}} \frac{d t_{2}}{t_{2}}\right\}^{1 / q}\right\|_{L^{p}\left(\mathbb{R}^{2}\right)}
$$

for $p \in(1, \infty)$ and $q \in(1, \infty]$, where the usual modifications are made when $p=\infty$ or $q=\infty$.

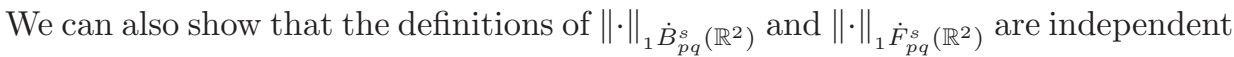
of the choices of $\psi^{(i)}$ for $i=1,2$ by an argument similar to that of Theorem 2.6.

Theorem 2.20. Let $s_{1}, s_{2} \in(-1,1),\left|s_{1}+2 s_{2}\right|<1$, and $s=\left(s_{1}, s_{2}\right)$. The norm $\|\cdot\|_{1} \dot{B}_{p q}^{s}\left(\mathbb{R}^{2}\right)$ with $p, q \in[1, \infty]$ and the norm $\|\cdot\|_{1} \dot{F}_{p q}^{s}\left(\mathbb{R}^{2}\right)$ with $p \in(1, \infty)$ and $q \in(1, \infty]$ are independent of the choices of $\psi^{(i)}$ for $i=1,2$.

Proof. Let $\tilde{\psi}^{(i)}$ for $i=1,2$ be the same as in the proof of Theorem 2.6. We denote the corresponding norms, respectively, by $\|\cdot\|_{2} \dot{B}_{p q}^{s}\left(\mathbb{R}^{2}\right)$ and $\|\cdot\|_{2} \dot{F}_{p q}^{s}\left(\mathbb{R}^{2}\right)$. To prove the theorem, by symmetry, we only need to show that there exists a positive constant $C$ such that, for all $f \in \mathcal{S}_{\infty, F}\left(\mathbb{R}^{2}\right)^{\prime}$,

$$
\|f\|_{2 \dot{B}_{p q}^{s}\left(\mathbb{R}^{2}\right)} \leq C\|f\|_{1} \dot{B}_{p q}^{s}\left(\mathbb{R}^{2}\right)
$$


and

$$
\|f\|_{2} \dot{F}_{p q}^{s}\left(\mathbb{R}^{2}\right) \leq C\|f\|_{1} \dot{F}_{p q}^{s}\left(\mathbb{R}^{2}\right),
$$

which, with the same notation as in the proof of Theorem 2.6, are deduced from the following facts:

$$
\int_{0}^{\infty} \int_{0}^{\infty}\left(\frac{u_{1}}{t_{1}} \wedge \frac{t_{1}}{u_{1}}\right)\left(\frac{u_{2}}{t_{2}} \wedge \frac{t_{2}}{u_{2}}\right)\left(\frac{t_{1}}{u_{1}}\right)^{s_{1}}\left(\frac{t_{1}^{2}+t_{2}}{u_{1}^{2}+u_{2}}\right)^{s_{2}} \frac{d t_{1}}{t_{1}} \frac{d t_{2}}{t_{2}} \leq C
$$

and

$$
\int_{0}^{\infty} \int_{0}^{\infty}\left(\frac{u_{1}}{t_{1}} \wedge \frac{t_{1}}{u_{1}}\right)\left(\frac{u_{2}}{t_{2}} \wedge \frac{t_{2}}{u_{2}}\right)\left(\frac{t_{1}}{u_{1}}\right)^{s_{1}}\left(\frac{t_{1}^{2}+t_{2}}{u_{1}^{2}+u_{2}}\right)^{s_{2}} \frac{d u_{1}}{u_{1}} \frac{d u_{2}}{u_{2}} \leq C .
$$

The proofs of both facts are similar by symmetry and we only show (21). To this end, we consider two cases.

- Case 1: $u_{2} \leq u_{1}^{2}$. In this case, the left-hand side of (21) is equivalent to

$$
\begin{aligned}
\int_{0}^{\infty} \int_{0}^{\infty}\left(\frac{u_{1}}{t_{1}} \wedge \frac{t_{1}}{u_{1}}\right)\left(\frac{u_{2}}{t_{2}} \wedge \frac{t_{2}}{u_{2}}\right)\left(\frac{t_{1}}{u_{1}}\right)^{s_{1}}\left(\frac{t_{1}^{2}+t_{2}}{u_{1}^{2}}\right)^{s_{2}} \frac{d t_{1}}{t_{1}} \frac{d t_{2}}{t_{2}} \\
=\int_{0}^{\infty} \int_{0}^{t_{1}^{2}}\left(\frac{u_{1}}{t_{1}} \wedge \frac{t_{1}}{u_{1}}\right)\left(\frac{u_{2}}{t_{2}} \wedge \frac{t_{2}}{u_{2}}\right)\left(\frac{t_{1}}{u_{1}}\right)^{s_{1}}\left(\frac{t_{1}^{2}+t_{2}}{u_{1}^{2}}\right)^{s_{2}} \frac{d t_{1}}{t_{1}} \frac{d t_{2}}{t_{2}} \\
+\int_{0}^{\infty} \int_{t_{1}^{2}}^{\infty} \cdots=P_{1}+P_{2} .
\end{aligned}
$$

For $P_{1}$, since $t_{1}^{2}+t_{2} \sim t_{1}^{2}$, we then have

$$
P_{1} \leq C \int_{0}^{\infty} \int_{0}^{\infty}\left(\frac{u_{1}}{t_{1}} \wedge \frac{t_{1}}{u_{1}}\right)\left(\frac{u_{2}}{t_{2}} \wedge \frac{t_{2}}{u_{2}}\right)\left(\frac{t_{1}}{u_{1}}\right)^{s_{1}+2 s_{2}} \frac{d t_{1}}{t_{1}} \frac{d t_{2}}{t_{2}} \leq C,
$$

by the assumption that $\left|s_{1}+2 s_{2}\right|<1$. For $P_{2}$, since $t_{1}^{2}+t_{2} \sim t_{2}$, we have

$$
P_{2} \leq C \int_{0}^{\infty} \int_{t_{1}^{2}}^{\infty}\left(\frac{u_{1}}{t_{1}} \wedge \frac{t_{1}}{u_{1}}\right)\left(\frac{u_{2}}{t_{2}} \wedge \frac{t_{2}}{u_{2}}\right)\left(\frac{t_{1}}{u_{1}}\right)^{s_{1}}\left(\frac{t_{2}}{u_{1}^{2}}\right)^{s_{2}} \frac{d t_{1}}{t_{1}} \frac{d t_{2}}{t_{2}} .
$$

If $s_{2} \leq 0$, the fact that $t_{2} \geq t_{1}^{2}$ and the assumption that $\left|s_{1}+2 s_{2}\right|<1$ imply the desired estimate of $P_{2}$; and if $s_{2}>0$, the assumptions that $u_{1}^{2} \geq u_{2}$ and $s_{1}, s_{2} \in(-1,1)$ also imply the desired estimate of $P_{2}$, which completes the proof of case 1 . 
- Case 2: $u_{1}^{2}<u_{2}$. In this case, the left-hand side of (21) is equivalent to

$$
\begin{aligned}
\int_{0}^{\infty} \int_{0}^{\infty}\left(\frac{u_{1}}{t_{1}} \wedge \frac{t_{1}}{u_{1}}\right)\left(\frac{u_{2}}{t_{2}} \wedge \frac{t_{2}}{u_{2}}\right)\left(\frac{t_{1}}{u_{1}}\right)^{s_{1}}\left(\frac{t_{1}^{2}+t_{2}}{u_{2}}\right)^{s_{2}} \frac{d t_{1}}{t_{1}} \frac{d t_{2}}{t_{2}} \\
\leq C \int_{0}^{\infty} \int_{0}^{t_{1}^{2}}\left(\frac{u_{1}}{t_{1}} \wedge \frac{t_{1}}{u_{1}}\right)\left(\frac{u_{2}}{t_{2}} \wedge \frac{t_{2}}{u_{2}}\right)\left(\frac{t_{1}}{u_{1}}\right)^{s_{1}}\left(\frac{t_{1}^{2}}{u_{2}}\right)^{s_{2}} \frac{d t_{1}}{t_{1}} \frac{d t_{2}}{t_{2}} \\
\quad+C \int_{0}^{\infty} \int_{t_{1}^{2}}^{\infty}\left(\frac{u_{1}}{t_{1}} \wedge \frac{t_{1}}{u_{1}}\right)\left(\frac{u_{2}}{t_{2}} \wedge \frac{t_{2}}{u_{2}}\right)\left(\frac{t_{1}}{u_{1}}\right)^{s_{1}}\left(\frac{t_{2}}{u_{2}}\right)^{s_{2}} \frac{d t_{1}}{t_{1}} \frac{d t_{2}}{t_{2}} .
\end{aligned}
$$

We can obtain the desired estimate for the second term of the last expression by the assumptions that $s_{1}, s_{2} \in(-1,1)$. For the first term, if $s_{2} \geq 0$, the fact that $u_{1}^{2}<u_{2}$ and the assumption that $\left|s_{1}+2 s_{2}\right|<1$ can imply the desired estimate; and if $s_{2}<0$, the fact that $t_{2}<t_{1}^{2}$ and the assumptions that $s_{1}, s_{2} \in(-1,1)$ also yield the desired estimate. This finishes the proof of (21) and hence, the proof of Theorem 2.20.

From Lemma 2.3 and Lemma 2.4, by an argument similar to the proof of Theorem 2.20, we also deduce the new 'discrete' characterization of Besov spaces $\dot{B}_{p q}^{s}\left(\mathbb{R}^{2}\right)$ and Triebel-Lizorkin spaces $\dot{F}_{p q}^{s}\left(\mathbb{R}^{2}\right)$ as follows. We omit the details.

Theorem 2.21. Let $s_{1}, s_{2} \in(-1,1),\left|s_{1}+2 s_{2}\right|<1, s=\left(s_{1}, s_{2}\right)$ and all other notation be the same as in Lemma 2.4. Then $f \in \dot{B}_{p q}^{s}\left(\mathbb{R}^{2}\right)$ with $p, q \in[1, \infty]$ if and only if $f \in \mathcal{S}_{\infty, F}\left(\mathbb{R}^{2}\right)^{\prime}$ and

$$
\begin{aligned}
\|f\|_{\dot{B}_{p q}^{s}\left(\mathbb{R}^{2}\right)}^{\prime \prime} & =\left\{\sum_{k_{1}=-\infty}^{\infty} \sum_{k_{2}=-\infty}^{\infty} 2^{k_{1} s_{1} q}\left(2^{-2 k_{1}}+2^{-k_{2}}\right)^{-s_{2} q}\left\|\psi_{k_{1} k_{2}} * f\right\|_{L^{p}\left(\mathbb{R}^{2}\right)}^{q}\right\}^{1 / q}<\infty,
\end{aligned}
$$

and $f \in \dot{F}_{p q}^{s}\left(\mathbb{R}^{2}\right)$ with $p \in(1, \infty)$ and $q \in(1, \infty]$ if and only if $f \in \mathcal{S}_{\infty, F}\left(\mathbb{R}^{2}\right)^{\prime}$ and

$$
\begin{aligned}
& \|f\|_{\dot{F}_{p q}^{s}\left(\mathbb{R}^{2}\right)}^{\prime \prime} \\
& \quad=\left\|\left\{\sum_{k_{1}=-\infty}^{\infty} \sum_{k_{2}=-\infty}^{\infty} 2^{k_{1} s_{1} q}\left(2^{-2 k_{1}}+2^{-k_{2}}\right)^{-s_{2} q}\left|\psi_{k_{1} k_{2}} * f\right|^{q}\right\}^{1 / q}\right\|_{L^{p}\left(\mathbb{R}^{2}\right)}<\infty .
\end{aligned}
$$

Furthermore, in this case, $\|\cdot\|_{\dot{B}_{p q}^{s}\left(\mathbb{R}^{2}\right)}^{\prime \prime}$ and $\|\cdot\|_{\dot{F}_{p q}^{s}\left(\mathbb{R}^{2}\right)}^{\prime \prime}$ are equivalent to $\|\cdot\|_{1} \dot{B}_{p q}^{s}\left(\mathbb{R}^{2}\right)$ and $\|\cdot\|_{1} \dot{F}_{p q}^{s}\left(\mathbb{R}^{2}\right)$, respectively.

As a corollary of Theorem 2.20 and Theorem 2.6, we obtain a new characterization of Besov spaces $\dot{B}_{p q}^{s}\left(\mathbb{R}^{2}\right)$ and Triebel-Lizorkin spaces $\dot{F}_{p q}^{s}\left(\mathbb{R}^{2}\right)$ as below, which clearly reveals the difference between $\dot{B}_{p q}^{s}\left(\mathbb{R}^{2}\right)$ and $\dot{F}_{p q}^{s}\left(\mathbb{R}^{2}\right)$ with the classical product Besov spaces and Triebel-Lizorkin spaces in [7]. 
Corollary 2.22. Let $s_{1}, s_{2} \in(-1,1),\left|s_{1}+2 s_{2}\right|<1$, and $s=\left(s_{1}, s_{2}\right)$. Then

(i) For $f \in \mathcal{S}_{\infty, F}\left(\mathbb{R}^{2}\right)^{\prime}, f \in \dot{B}_{p q}^{s}\left(\mathbb{R}^{2}\right)$ with $p, q \in[1, \infty]$ if and only if $\|f\|_{1} \dot{B}_{p q}^{s}\left(\mathbb{R}^{2}\right)$ $<\infty$. Moreover, in this case, $\|f\|_{\dot{B}_{p q}^{s}\left(\mathbb{R}^{2}\right)} \sim\|f\|_{1 \dot{B}_{p q}^{s}\left(\mathbb{R}^{2}\right)}$.

(ii) For $f \in \mathcal{S}_{\infty, F}\left(\mathbb{R}^{2}\right)^{\prime}, f \in \dot{F}_{p q}^{s}\left(\mathbb{R}^{2}\right)$ with $p \in(1, \infty)$ and $q \in(1, \infty]$ if and only if

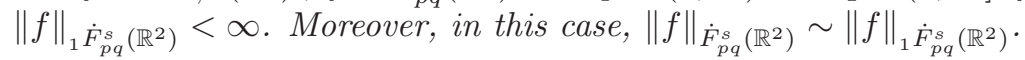

Proof. Theorem 2.6 and Theorem 2.20 imply that the definitions of the norms $\|\cdot\|_{\dot{B}_{p q}^{s}\left(\mathbb{R}^{2}\right)},\|\cdot\|_{1 \dot{B}_{p q}^{s}\left(\mathbb{R}^{2}\right)},\|\cdot\|_{\dot{F}_{p q}^{s}\left(\mathbb{R}^{2}\right)}$, and $\|\cdot\|_{1 \dot{F}_{p q}^{s}\left(\mathbb{R}^{2}\right)}$ are independent of the choices of $\psi^{(i)}$ for $i=1,2$. Let $\psi^{(i)}$ for $i=1,2$ be the same as in Lemma 2.3 satisfying the following additional conditions:

and

$$
\operatorname{supp} \hat{\psi}^{(1)} \subset\left\{\left(\xi_{1}, \xi_{2}\right) \in \mathbb{R}^{2}: 1 / 2 \leq\left\|\left(\xi_{1}, \xi_{2}\right)\right\| \leq 2\right\}
$$

$$
\operatorname{supp} \hat{\psi}^{(2)} \subset\{\xi \in \mathbb{R}: 1 / 2 \leq|\xi| \leq 2\} .
$$

From the definitions of $\psi_{t_{1} t_{2}}$, the above conditions of the supports of $\psi^{(i)}$ with $i=1,2$, and the Plancherel principle, it is easy to deduce that $\psi_{t_{1} t_{2}}=0$ if $t_{1}^{2} \leq 8 t_{2}$. Thus, using such $\psi_{t_{1} t_{2}}$ and noticing that $t_{1}^{2}+t_{2} \sim t_{2}$ if $t_{1}^{2} \leq 8 t_{2}$, by Definitions 2.5 and 2.19, we easily obtain that $\|f\|_{\dot{B}_{p q}^{s}\left(\mathbb{R}^{2}\right)} \sim\|f\|_{1 \dot{B}_{p q}^{s}\left(\mathbb{R}^{2}\right)}$ and $\|f\|_{\dot{F}_{p q}^{s}\left(\mathbb{R}^{2}\right)} \sim\|f\|_{1 \dot{F}_{p q}^{s}\left(\mathbb{R}^{2}\right)}$. This finishes the proof of Corollary 2.22 .

\section{Boundedness of flag singular integrals}

We now establish the boundedness on $\dot{B}_{p q}^{s}\left(\mathbb{R}^{2}\right)$ and $\dot{F}_{p q}^{s}\left(\mathbb{R}^{2}\right)$ of flag singular integrals.

Since it is well-known that the flag singular integral is bounded on $L^{p}\left(\mathbb{R}^{2}\right)$ for $p \in(1, \infty)$, we then automatically deduce that it is also bounded on classical Besov spaces and the new Besov spaces $\dot{B}_{p q}^{s}\left(\mathbb{R}^{2}\right)$ associated with flag kernels, when $p \in(1, \infty)$. However, this is not true for Besov spaces when $p=1$ or $p=\infty$ and Triebel-Lizorkin spaces. Moreover, our argument also gives a direct proof for the boundedness of flag singular integrals in $L^{p}\left(\mathbb{R}^{2}\right)$ with $p \in(1, \infty)$.

Theorem 3.1. Let $K$ be an integrable flag kernel on $\mathbb{R}^{2}$ as in Definition 1.2, and $s=\left(s_{1}, s_{2}\right)$ with $s_{i} \in(-1,1)$ for $i=1,2$. Then

(i) If $p, q \in[1, \infty]$, then there exists a positive constant $C$ such that, for all $f \in \dot{B}_{p q}^{s}\left(\mathbb{R}^{2}\right)$,

$$
\|K * f\|_{\dot{B}_{p q}^{s}\left(\mathbb{R}^{2}\right)} \leq C\|f\|_{\dot{B}_{p q}^{s}\left(\mathbb{R}^{2}\right)} .
$$

(ii) If $p \in(1, \infty)$ and $q \in(1, \infty]$, then there exists a positive constant $C$ such that, for all $f \in \dot{F}_{p q}^{s}\left(\mathbb{R}^{2}\right)$,

$$
\|K * f\|_{\dot{F}_{p q}^{s}\left(\mathbb{R}^{2}\right)} \leq C\|f\|_{\dot{F}_{p q}^{s}\left(\mathbb{R}^{2}\right)} .
$$


Before proving Theorem 3.1, we first establish several lemmas which are used in the proof of Theorem 3.1.

Lemma 3.2. Let $K$ be a distribution on $\mathbb{R}$ which is a continuous function on $\Omega_{1}=\mathbb{R} \backslash\{0\}$ and $\psi_{s}$ be a function on $\mathbb{R}$ for all $s>0$. Suppose that there exists a positive constant $C_{K}$ such that $K$ satisfies the following conditions:

(i) For all $x_{3} \in \Omega_{1},\left|K\left(x_{3}\right)\right| \leq C_{K} \frac{1}{\left|x_{3}\right|}$.

(ii) For all $x_{3} \in \Omega_{1}$ and $x_{3}^{\prime} \in \mathbb{R}$ with $\left|x_{3}-x_{3}^{\prime}\right| \leq\left|x_{3}\right| / 2$,

$$
\left|K\left(x_{3}\right)-K\left(x_{3}^{\prime}\right)\right| \leq C_{K} \frac{\left|x_{3}-x_{3}^{\prime}\right|}{\left|x_{3}\right|^{2}} .
$$

(iii) For any given bump function $\varphi$ on $\mathbb{R}$ and any $\delta>0$,

$$
\left|\int_{\mathbb{R}} K\left(x_{3}\right) \varphi\left(\delta x_{3}\right) d x_{3}\right| \leq C_{K}
$$

Suppose also that there exists a positive constant $C_{\psi}$ such that

(iv) For all $s>0$ and $x_{3} \in \mathbb{R},\left|\psi_{s}\left(x_{3}\right)\right| \leq C_{\psi} \frac{s}{\left(s+\left|x_{3}\right|\right)^{2}}$.

(v) For all $s>0$ and $x_{3}, x_{3}^{\prime} \in \mathbb{R}$ with $\left|x_{3}-x_{3}^{\prime}\right| \leq\left(s+\left|x_{3}\right|\right) / 2$,

$$
\left|\psi_{s}\left(x_{3}\right)-\psi_{s}\left(x_{3}^{\prime}\right)\right| \leq C_{\psi} \frac{\left|x_{3}-x_{3}^{\prime}\right|}{s+\left|x_{3}\right|} \cdot \frac{s}{\left(s+\left|x_{3}\right|\right)^{2}}
$$

(vi) For all $s>0, \int_{\mathbb{R}} \psi_{s}\left(x_{3}\right) d x_{3}=0$.

(vii) $\operatorname{supp} \psi_{s} \subset\left\{x_{3} \in \mathbb{R}:\left|x_{3}\right| \leq s\right\}$.

Then there exists a positive constant $C$ such that, for all $s>0$ and $z \in \mathbb{R}$,

$$
\left|\int_{\mathbb{R}} K\left(z-x_{3}\right) \psi_{s}\left(x_{3}\right) d x_{3}\right| \leq C C_{K} C_{\psi} \frac{s}{(s+|z|)^{2}}
$$

Proof. We consider two cases.

- Case 1: $|z| \leq 5 s$. In this case, let $\theta \in C_{0}^{\infty}(\mathbb{R}), 0 \leq \theta \leq 1, \operatorname{supp} \theta \subset\{x \in \mathbb{R}$ : $|x| \leq 2\}$, and $\theta(x)=1$ if $|x| \leq 1$. We then define $\xi(z)=\theta\left(\frac{|z|}{10 s}\right)$ for $z \in \mathbb{R}$. By (vii), 
we can write

$$
\begin{aligned}
\left|\int_{\mathbb{R}} K\left(z-x_{3}\right) \psi_{s}\left(x_{3}\right) d x_{3}\right| & \\
\leq & \int_{\left|x_{3}-z\right| \leq(s+|z|) / 2}\left|K\left(z-x_{3}\right)\left[\psi_{s}\left(x_{3}\right)-\psi_{s}(z)\right]\right| \xi\left(x_{3}\right) d x_{3} \\
& +\int_{\left|x_{3}-z\right|>(s+|z|) / 2} \cdots+\left|\psi_{s}(z)\right|\left|\int_{\mathbb{R}} K\left(z-x_{3}\right) \xi\left(x_{3}\right) d x_{3}\right| \\
= & G_{1}+G_{2}+G_{3} .
\end{aligned}
$$

Notice that $\xi$ is a normalized bump function multiplied with a normalizing constant and some dilation. The assumptions (iii) and (iv) give us the desired estimate for $G_{3}$ :

$$
G_{3} \leq C_{K} C_{\psi} \frac{s}{(s+|z|)^{2}}
$$

From (i) and (v), it follows that

$$
G_{1} \leq C_{K} C_{\psi} \int_{\mathbb{R}} \frac{1}{\left|z-x_{3}\right|} \frac{\left|x_{3}-z\right|}{s+\left|x_{3}\right|} \frac{s}{\left(s+\left|x_{3}\right|\right)^{2}} d x_{3} \leq C C_{K} C_{\psi} \frac{1}{s} .
$$

The assumptions (i) and (iv) also yield that

$$
\begin{aligned}
G_{2} & \leq \int_{\left|x_{3}-z\right|>(s+|z|) / 2}\left|K\left(z-x_{3}\right)\right|\left[\left|\psi_{s}\left(x_{3}\right)\right|+\left|\psi_{s}(z)\right|\right] \xi\left(x_{3}\right) d x_{3} \\
& \leq C C_{K} \frac{1}{s}\left[\int_{\mathbb{R}}\left|\psi_{s}\left(x_{3}\right)\right| d x_{3}+C_{\psi} \frac{s^{2}}{(s+|z|)^{2}}\right] \leq C C_{K} C_{\psi} \frac{s}{(s+|z|)^{2}},
\end{aligned}
$$

which completes the proof of case 1 .

- Case 2: $|z|>5 s$. In this case, by (vi), (vii), (ii), and (iv), we have

$$
\begin{aligned}
\left|\int_{\mathbb{R}} K\left(z-x_{3}\right) \psi_{s}\left(x_{3}\right) d x_{3}\right| & =\left|\int_{\mathbb{R}}\left[K\left(z-x_{3}\right)-K(z)\right] \psi_{s}\left(x_{3}\right) d x_{3}\right| \\
& \leq C_{K} \int_{\mathbb{R}} \frac{\left|x_{3}\right|}{|z|^{2}}\left|\psi_{s}\left(x_{3}\right)\right| d x_{3} \leq C_{K} C_{\psi} \frac{s}{|z|^{2}} .
\end{aligned}
$$

This finishes the proof of Lemma 3.2 .

The same argument as in the proof of Lemma 3.2 gives us the following result and we omit the details.

Lemma 3.3. Let $K$ be a distribution on $\mathbb{R}^{2}$ which is a continuous function on $\Omega_{2}=\mathbb{R}^{2} \backslash\{(0,0)\}$ and $\psi_{s}$ be a function on $\mathbb{R}^{2}$ for all $s>0$. Suppose that there exists a positive constant $C_{K}$ such that $K$ satisfies the following conditions: 
(i) For all $\left(x_{1}, x_{2}\right) \in \Omega_{2},\left|K\left(x_{1}, x_{2}\right)\right| \leq C_{K} \frac{1}{\left\|\left(x_{1}, x_{2}\right)\right\|^{3}}$.

(ii) For all $\left(x_{1}, x_{2}\right) \in \Omega_{2}$ and $\left(x_{1}^{\prime}, x_{2}^{\prime}\right) \in \mathbb{R}^{2}$ with $\left\|\left(x_{1}, x_{2}\right)-\left(x_{1}^{\prime}, x_{2}^{\prime}\right)\right\| \leq\left\|\left(x_{1}, x_{2}\right)\right\| / 2$,

$$
\left|K\left(x_{1}, x_{2}\right)-K\left(x_{1}^{\prime}, x_{2}^{\prime}\right)\right| \leq C_{K} \frac{\left\|\left(x_{1}, x_{2}\right)-\left(x_{1}^{\prime}, x_{2}^{\prime}\right)\right\|}{\left\|\left(x_{1}, x_{2}\right)\right\|^{4}} .
$$

(iii) For any given bump function $\varphi$ on $\mathbb{R}^{2}$ and any $\delta>0$,

$$
\left|\int_{\mathbb{R}^{2}} K\left(x_{1}, x_{2}\right) \varphi\left(\delta x_{1}, \delta^{2} x_{2}\right) d x_{1} d x_{2}\right| \leq C_{K}
$$

Suppose also that there exists a positive constant $C_{\psi}$ such that

(iv) For all $s>0$ and $\left(x_{1}, x_{2}\right) \in \mathbb{R}^{2},\left|\psi_{s}\left(x_{1}, x_{2}\right)\right| \leq C_{\psi} \frac{s}{\left(s+\left\|\left(x_{1}, x_{2}\right)\right\|\right)^{4}}$.

(v) For all $s>0$ and $\left(x_{1}, x_{2}\right),\left(x_{1}^{\prime}, x_{2}^{\prime}\right) \in \mathbb{R}^{2}$ with $\left\|\left(x_{1}, x_{2}\right)-\left(x_{1}^{\prime}, x_{2}^{\prime}\right)\right\| \leq$ $\left(s+\left\|\left(x_{1}, x_{2}\right)\right\|\right) / 2$,

$$
\left|\psi_{s}\left(x_{1}, x_{2}\right)-\psi_{s}\left(x_{1}^{\prime}, x_{2}^{\prime}\right)\right| \leq C_{\psi} \frac{\left\|\left(x_{1}, x_{2}\right)-\left(x_{1}^{\prime}, x_{2}^{\prime}\right)\right\|}{s+\left\|\left(x_{1}, x_{2}\right)\right\|} \cdot \frac{s}{\left(s+\left\|\left(x_{1}, x_{2}\right)\right\|\right)^{4}} .
$$

(vi) For all $s>0, \int_{\mathbb{R}^{2}} \psi_{s}\left(x_{1}, x_{2}\right) d x_{1} d x_{2}=0$.

(vii) $\operatorname{supp} \psi_{s} \subset\left\{\left(x_{1}, x_{2}\right) \in \mathbb{R}:\left\|\left(x_{1}, x_{2}\right)\right\| \leq s\right\}$.

Then there exists a positive constant $C$ such that, for all $s>0$ and $z \in \mathbb{R}$,

$$
\left|\int_{\mathbb{R}^{2}} K\left(x_{1}-y_{1}, x_{2}-y_{2}\right) \psi_{s}\left(y_{1}, y_{2}\right) d y_{1} d y_{2}\right| \leq C C_{K} C_{\psi} \frac{s}{\left(s+\left\|\left(x_{1}, x_{2}\right)\right\|\right)^{4}} .
$$

We also need the following basic estimate.

Lemma 3.4. Let $\psi^{(1)} \in C_{0}^{\infty}\left(\mathbb{R}^{2}\right), \operatorname{supp} \psi^{(1)} \subset\left\{\left(x_{1}, x_{2}\right) \in \mathbb{R}^{2}:\left\|\left(x_{1}, x_{2}\right)\right\|<1\right\}$ and

$$
\int_{\mathbb{R}^{2}} \psi^{(1)}\left(x_{1}, x_{2}\right) d x_{1} d x_{2}=0 .
$$

For $s>0$ and $\left(x_{1}, x_{2}\right) \in \mathbb{R}^{2}$, set $\psi_{s}^{(1)}\left(x_{1}, x_{2}\right)=\frac{1}{s^{3}} \psi^{(1)}\left(\frac{x_{1}}{s}, \frac{x_{2}}{s^{2}}\right)$. Then there exists a positive constant $C$ such that

(i) For all $t_{1}, u_{1}>0$ and $\left(x_{1}, x_{2}\right) \in \mathbb{R}^{2}$,

$$
\left|\left(\psi_{t_{1}}^{(1)} * \psi_{u_{1}}^{(1)}\right)\left(x_{1}, x_{2}\right)\right| \leq C\left(\frac{u_{1}}{t_{1}} \wedge \frac{t_{1}}{u_{1}}\right) \frac{t_{1} \vee u_{1}}{\left(t_{1} \vee u_{1}+\left\|\left(x_{1}, x_{2}\right)\right\|\right)^{4}} .
$$


(ii) For all $t_{1}, u_{1}>0$ and $\left(x_{1}, x_{2}\right),\left(y_{1}, y_{2}\right) \in \mathbb{R}^{2}$ with $\left\|\left(y_{1}, y_{2}\right)-\left(x_{1}, x_{2}\right)\right\| \leq$ $\left(t_{1} \vee u_{1}+\left\|\left(x_{1}, x_{2}\right)\right\|\right) / 2$,

$$
\begin{aligned}
& \mid\left(\psi_{t_{1}}^{(1)} * \psi_{u_{1}}^{(1)}\right)\left(y_{1}, y_{2}\right)-\left(\psi_{t_{1}}^{(1)} * \psi_{u_{1}}^{(1)}\right)\left(x_{1}, x_{2}\right) \mid \\
& \leq C\left(\frac{u_{1}}{t_{1}} \wedge \frac{t_{1}}{u_{1}}\right) \frac{\left\|\left(y_{1}, y_{2}\right)-\left(x_{1}, x_{2}\right)\right\|}{t_{1} \vee u_{1}+\left\|\left(x_{1}, x_{2}\right)\right\|} \cdot \frac{t_{1} \vee u_{1}}{\left(t_{1} \vee u_{1}+\left\|\left(x_{1}, x_{2}\right)\right\|\right)^{4}} .
\end{aligned}
$$

Proof. The same argument as in the proof of (7) also yields (i). We only show (ii) in the case $u_{1} \leq t_{1}$. In this case, the estimate (ii) becomes that if $\left\|\left(y_{1}, y_{2}\right)-\left(x_{1}, x_{2}\right)\right\| \leq$ $\left(t_{1}+\left\|\left(x_{1}, x_{2}\right)\right\|\right) / 2$ then

$$
\begin{aligned}
& \left|\left(\psi_{t_{1}}^{(1)} * \psi_{u_{1}}^{(1)}\right)\left(y_{1}, y_{2}\right)-\left(\psi_{t_{1}}^{(1)} * \psi_{u_{1}}^{(1)}\right)\left(x_{1}, x_{2}\right)\right| \\
& \quad \leq C \frac{u_{1}}{t_{1}} \frac{\left\|\left(y_{1}, y_{2}\right)-\left(x_{1}, x_{2}\right)\right\|}{t_{1}+\left\|\left(x_{1}, x_{2}\right)\right\|} \cdot \frac{t_{1}}{\left(t_{1}+\left\|\left(x_{1}, x_{2}\right)\right\|\right)^{4}} .
\end{aligned}
$$

To guarantee

$$
\left|\left(\psi_{t_{1}}^{(1)} * \psi_{u_{1}}^{(1)}\right)\left(y_{1}, y_{2}\right)-\left(\psi_{t_{1}}^{(1)} * \psi_{u_{1}}^{(1)}\right)\left(x_{1}, x_{2}\right)\right| \neq 0
$$

when $\left\|\left(y_{1}, y_{2}\right)-\left(x_{1}, x_{2}\right)\right\| \leq\left(t_{1}+\left\|\left(x_{1}, x_{2}\right)\right\|\right) / 2$, we always have $\left\|\left(x_{1}, x_{2}\right)\right\| \leq C t_{1}$. Thus, by (22) and the mean value theorem,

$$
\begin{aligned}
\mid\left(\psi_{t_{1}}^{(1)} *\right. & \left.\psi_{u_{1}}^{(1)}\right)\left(y_{1}, y_{2}\right)-\left(\psi_{t_{1}}^{(1)} * \psi_{u_{1}}^{(1)}\right)\left(x_{1}, x_{2}\right) \mid \\
= & \mid \int_{\mathbb{R}^{2}}\left\{\left[\psi_{t_{1}}^{(1)}\left(y_{1}-z_{1}, y_{2}-z_{2}\right)-\psi_{t_{1}}^{(1)}\left(x_{1}-z_{1}, x_{2}-z_{2}\right)\right]\right. \\
& \left.-\left[\psi_{t_{1}}^{(1)}\left(y_{1}, y_{2}\right)-\psi_{t_{1}}^{(1)}\left(x_{1}, x_{2}\right)\right]\right\} \psi_{u_{1}}^{(1)}\left(z_{1}, z_{2}\right) d z_{1} d z_{2} \mid \\
\leq & C \frac{u_{1}}{t_{1}^{5}}\left\|\left(y_{1}, y_{2}\right)-\left(x_{1}, x_{2}\right)\right\| \leq C \frac{u_{1}}{t_{1}} \frac{\left\|\left(y_{1}, y_{2}\right)-\left(x_{1}, x_{2}\right)\right\|}{t_{1}+\left\|\left(x_{1}, x_{2}\right)\right\|} \frac{t_{1}}{\left(t_{1}+\left\|\left(x_{1}, x_{2}\right)\right\|\right)^{4}},
\end{aligned}
$$

which completes the proof of Lemma 3.4.

The same argument as in the proof of Lemma 3.4 also yields the following basic estimates and we omit the details.

Lemma 3.5. Let $\psi^{(2)} \in C_{0}^{\infty}(\mathbb{R}), \operatorname{supp} \psi^{(2)} \subset(-1,1)$, and $\int_{\mathbb{R}} \psi\left(x_{3}\right) d x_{3}=0$. For $s>0$ and $x_{3} \in \mathbb{R}$, set $\psi_{s}^{(2)}\left(x_{3}\right)=\frac{1}{s} \psi^{(2)}\left(\frac{x_{3}}{s}\right)$. Then there exists a positive constant $C$ such that

(i) For all $t_{2}, u_{2}>0$ and $x_{3} \in \mathbb{R}$,

$$
\left|\left(\psi_{t_{2}}^{(2)} * \psi_{u_{2}}^{(2)}\right)\left(x_{3}\right)\right| \leq C\left(\frac{u_{2}}{t_{2}} \wedge \frac{t_{2}}{u_{2}}\right) \frac{t_{2} \vee u_{2}}{\left(t_{2} \vee u_{2}+\left|x_{3}\right|\right)^{2}} .
$$


(ii) For all $t_{2}, u_{2}>0$ and $x_{3}, y_{3} \in \mathbb{R}$ with $\left|y_{3}-x_{3}\right| \leq\left(t_{2} \vee u_{2}+\left|x_{3}\right|\right) / 2$,

$$
\begin{aligned}
\left|\left(\psi_{t_{2}}^{(2)} * \psi_{u_{2}}^{(2)}\right)\left(y_{3}\right)-\left(\psi_{t_{2}}^{(2)} * \psi_{u_{2}}^{(2)}\right)\left(x_{3}\right)\right| & \\
& \leq C\left(\frac{u_{2}}{t_{2}} \wedge \frac{t_{2}}{u_{2}}\right) \frac{\left|y_{3}-x_{3}\right|}{t_{2} \vee u_{2}+\left|x_{3}\right|} \cdot \frac{t_{2} \vee u_{2}}{\left(t_{2} \vee u_{2}+\left|x_{3}\right|\right)^{2}} .
\end{aligned}
$$

Lemma 3.6. Let $K^{\sharp}$ be a product kernel on $\mathbb{R}^{2} \times \mathbb{R}$ and $\psi_{s}$ be the same as in Lemma 3.2. For $\left(x_{1}, x_{2}\right) \in \mathbb{R}^{2}$ and $z \in \mathbb{R}$, define

$$
\tilde{K}\left(x_{1}, x_{2}, x_{3}\right)=\int_{\mathbb{R}^{2}} K^{\sharp}\left(x_{1}, x_{2}, x_{3}-z\right) \psi_{s}(z) d z .
$$

Then $\tilde{K}$ satisfies the same conditions of $K$ on $\left(x_{1}, x_{2}\right) \in \mathbb{R}^{2}$ as in Lemma 3.3 with $C_{\tilde{K}}$ no more than $C_{\psi} \frac{s}{\left(s+\left|x_{3}\right|\right)^{2}}$.

Proof. For any fixed $\left(x_{1}, x_{2}\right) \in \Omega_{2}$, by Definition 1.1, it is easy to see that $K^{\sharp}\left(x_{1}, x_{2}, \cdot\right)$ satisfies all the conditions of Lemma 3.2 with $C_{K^{\sharp}\left(x_{1}, x_{2}, \cdot\right)} \leq C_{\frac{1}{\left\|\left(x_{1}, x_{2}\right)\right\|^{3}}}$. Thus, Lemma 3.2 yields that, for all $s>0,\left(x_{1}, x_{2}\right) \in \Omega_{2}$, and $x_{3} \in \mathbb{R}$,

$$
\left|\tilde{K}\left(x_{1}, x_{2}, x_{3}\right)\right| \leq C C_{\psi} \frac{s}{\left(s+\left|x_{3}\right|\right)^{2}} \frac{1}{\left\|\left(x_{1}, x_{2}\right)\right\|^{3}},
$$

which shows that for any fixed $x_{3} \in \mathbb{R}, \tilde{K}\left(\cdot{ }_{1}, \cdot 2, x_{3}\right)$ satisfies Lemma 3.3 (i).

We now show that $\tilde{K}$ satisfies Lemma 3.3 (ii). Let $\left\|\left(x_{1}, x_{2}\right)-\left(y_{1}, y_{2}\right)\right\| \leq$ $\left\|\left(x_{1}, x_{2}\right)\right\| / 2$. By the mean value theorem and Definition 1.1, we have that

$$
\begin{aligned}
&\left|K^{\sharp}\left(x_{1}, x_{2}, x_{3}\right)-K^{\sharp}\left(y_{1}, y_{2}, x_{3}\right)\right| \\
& \leq C \frac{1}{\left\|\left((1-\kappa) x_{1}+\kappa y_{1},(1-\kappa) x_{2}+\kappa y_{2}\right)\right\|^{4}\left|x_{3}\right|} \\
& \times\left\{\left|x_{1}-y_{1}\right|+\frac{\left|x_{2}-y_{2}\right|}{\left\|\left((1-\kappa) x_{1}+\kappa y_{1},(1-\kappa) x_{2}+\kappa y_{2}\right)\right\|}\right\} \\
& \leq C \frac{\left\|\left(x_{1}, x_{2}\right)-\left(y_{1}, y_{2}\right)\right\|}{\left\|\left(x_{1}, x_{2}\right)\right\|^{4}\left|x_{3}\right|},
\end{aligned}
$$

where $\kappa \in(0,1)$ and we used the fact that

$$
\left\|\left((1-\kappa) x_{1}+\kappa y_{1},(1-\kappa) x_{2}+\kappa y_{2}\right)\right\| \geq\left\|\left(x_{1}, x_{2}\right)\right\| / 2 .
$$


Similarly, if $\left|x_{3}-y_{3}\right| \leq\left|x_{3}\right| / 2$, we have

$$
\begin{aligned}
\mid\left[K^{\sharp}\left(x_{1}, x_{2}, x_{3}\right)-\right. & \left.K^{\sharp}\left(y_{1}, y_{2}, x_{3}\right)\right]-\left[K^{\sharp}\left(x_{1}, x_{2}, y_{3}\right)-K^{\sharp}\left(y_{1}, y_{2}, y_{3}\right)\right] \mid \\
\leq & C \frac{\left|x_{3}-y_{3}\right|}{\left\|\left((1-\kappa) x_{1}+\kappa y_{1},(1-\kappa) x_{2}+\kappa y_{2}\right)\right\|^{4}\left|\left(1-\kappa_{0}\right) x_{3}+\kappa_{0} y_{3}\right|^{2}} \\
& \times\left\{\left|x_{1}-y_{1}\right|+\frac{\left|x_{2}-y_{2}\right|}{\left\|\left((1-\kappa) x_{1}+\kappa y_{1},(1-\kappa) x_{2}+\kappa y_{2}\right)\right\|}\right\} \\
\leq & C \frac{\left\|\left(x_{1}, x_{2}\right)-\left(y_{1}, y_{2}\right)\right\|\left|x_{3}-y_{3}\right|}{\left\|\left(x_{1}, x_{2}\right)\right\|^{4}\left|x_{3}\right|^{2}},
\end{aligned}
$$

where $\kappa, \kappa_{0} \in(0,1)$. Let $\varphi$ be a normalized bump function on $\mathbb{R}$. The mean value theorem and Definition 1.1 further yield that, for all $\delta>0$,

$$
\begin{aligned}
\left|\int_{\mathbb{R}}\left[K^{\sharp}\left(x_{1}, x_{2}, x_{3}\right)-K^{\sharp}\left(y_{1}, y_{2}, x_{3}\right)\right] \varphi\left(\delta x_{3}\right) d x_{3}\right| & \\
\leq & C \frac{1}{\left\|\left((1-\kappa) x_{1}+\kappa y_{1},(1-\kappa) x_{2}+\kappa y_{2}\right)\right\|^{4}} \\
& \times\left[\left|x_{1}-y_{1}\right|+\frac{\left|x_{2}-y_{2}\right|}{\left\|\left((1-\kappa) x_{1}+\kappa y_{1},(1-\kappa) x_{2}+\kappa y_{2}\right)\right\|}\right] \\
\leq & C \frac{\left\|\left(x_{1}, x_{2}\right)-\left(y_{1}, y_{2}\right)\right\|}{\left\|\left(x_{1}, x_{2}\right)\right\|^{4}},
\end{aligned}
$$

where $\kappa \in(0,1)$. Thus, for any fixed $\left(x_{1}, x_{2}\right) \in \Omega_{2}$ and any $\left(y_{1}, y_{2}\right) \in \mathbb{R}^{2}$ with $\left\|\left(x_{1}, x_{2}\right)-\left(y_{1}, y_{2}\right)\right\| \leq\left\|\left(x_{1}, x_{2}\right)\right\| / 2, K^{\sharp}\left(x_{1}, x_{2}, \cdot\right)-K^{\sharp}\left(y_{1}, y_{2}, \cdot\right)$ satisfies all the conditions of Lemma 3.2 with

$$
C_{K^{\sharp}\left(x_{1}, x_{2}, \cdot\right)-K^{\sharp}\left(y_{1}, y_{2}, \cdot\right)} \leq C \frac{\left\|\left(x_{1}, x_{2}\right)-\left(y_{1}, y_{2}\right)\right\|}{\left\|\left(x_{1}, x_{2}\right)\right\|^{4}} .
$$

Lemma 3.2 yields that

$$
\begin{aligned}
\left|\tilde{K}\left(x_{1}, x_{2}, x_{3}\right)-\tilde{K}\left(y_{1}, y_{2}, x_{3}\right)\right| & \\
& =\left|\int_{\mathbb{R}}\left[K^{\sharp}\left(x_{1}, x_{2}, x_{3}-z\right)-K^{\sharp}\left(y_{1}, y_{2}, x_{3}-z\right)\right] \psi_{s}(z) d z\right| \\
& \leq C C_{\psi} \frac{s}{\left(s+\left|x_{3}\right|\right)^{2}} \cdot \frac{\left\|\left(x_{1}, x_{2}\right)-\left(y_{1}, y_{2}\right)\right\|}{\left\|\left(x_{1}, x_{2}\right)\right\|^{4}},
\end{aligned}
$$

which shows that, for any fixed $x_{3} \in \mathbb{R}, \tilde{K}\left(\cdot_{1}, \cdot_{2}, x_{3}\right)$ satisfies Lemma 3.3 (ii).

Finally, let $\varphi$ be a normalized bump function on $\mathbb{R}^{2}$ and $\delta>0$. By the mean value theorem and Definition 1.1, we can easily show that

$$
\int_{\mathbb{R}^{2}} K^{\sharp}\left(x_{1}, x_{2}, \cdot\right) \varphi\left(\delta x_{1}, \delta^{2} x_{2}\right) d x_{1} d x_{2}
$$


satisfies all the conditions of Lemma 3.2 and hence

$$
\begin{aligned}
\mid \int_{\mathbb{R}^{2}} \tilde{K}\left(x_{1}, x_{2}, x_{3}\right) \varphi( & \left.\delta x_{1}, \delta^{2} x_{2}\right) d x_{1} d x_{2} \mid \\
& =\left|\int_{\mathbb{R}}\left[\int_{\mathbb{R}^{2}} K^{\sharp}\left(x_{1}, x_{2}, x_{3}-z\right) \varphi\left(\delta x_{1}, \delta^{2} x_{2}\right) d x_{1} d x_{2}\right] \psi_{s}(z) d z\right| \\
\leq & C C_{\psi} \frac{s}{\left(s+\left|x_{3}\right|\right)^{2}},
\end{aligned}
$$

which yields that, for any fixed $x_{3} \in \mathbb{R}, \tilde{K}\left(\cdot \cdot_{1}, \cdot_{2}, x_{3}\right)$ satisfies Lemma 3.3 (iii). This finishes the proof of Lemma 3.6.

Proof of Theorem 3.1. By Proposition 1.4, there exists an integrable product kernel $K^{\sharp}$ on $\mathbb{R}^{2} \times \mathbb{R}$ such that for all $\left(x_{1}, x_{2}\right) \in \mathbb{R}^{2}$,

$$
K\left(x_{1}, x_{2}\right)=\int_{\mathbb{R}} K^{\sharp}\left(x_{1}, x_{2}-x_{3}, x_{3}\right) d x_{3} .
$$

Let $\psi_{t_{1} t_{2}}$ be the same as in Lemma 2.3 ; moreover, let

$$
\operatorname{supp} \psi^{(1)} \subset\left\{\left(x_{1}, x_{2}\right):\left\|\left(x_{1}, x_{2}\right)\right\|<1\right\}
$$

and $\operatorname{supp} \psi^{(2)} \subset(-1,1)$. By Lemma 2.3, for $u_{1}, u_{2}>0$ and $\left(x_{1}, x_{2}\right) \in \mathbb{R}^{2}$, we have

$$
\begin{aligned}
& \left(\psi_{u_{1} u_{2}} * K * f\right)\left(x_{1}, x_{2}\right) \\
& =\int_{0}^{\infty} \int_{0}^{\infty}\left(\psi_{u_{1} u_{2}} * K * \psi_{t_{1} t_{2}} * \psi_{t_{1} t_{2}} * f\right)\left(x_{1}, x_{2}\right) \frac{d t_{1}}{t_{1}} \frac{d t_{2}}{t_{2}}
\end{aligned}
$$

and

$$
\begin{aligned}
\psi_{u_{1} u_{2}} * K * \psi_{t_{1} t_{2}}( & \left.x_{1}, x_{2}\right) \\
= & \int_{\mathbb{R}} \int_{\mathbb{R}^{2}}\left(\psi_{u_{1}}^{(1)} * \psi_{t_{1}}^{(1)}\right)\left(x_{1}-y_{1}, x_{2}-x_{3}-y_{2}\right) \\
& \times\left[\int_{\mathbb{R}} K^{\sharp}\left(y_{1}, y_{2}, x_{3}-y_{3}\right)\left(\psi_{u_{2}}^{(2)} * \psi_{t_{2}}^{(2)}\right)\left(y_{3}\right) d y_{3}\right] d y_{1} d y_{2} d x_{3} .
\end{aligned}
$$

Lemma 3.5 implies that $\psi_{u_{2}}^{(2)} * \psi_{t_{2}}^{(2)}$ satisfies all the conditions of Lemma 3.2 with $s=u_{2} \vee t_{2}$ and

$$
C_{\psi_{u_{2}}^{(2)} * \psi_{t_{2}}^{(2)}} \leq C\left(\frac{u_{2}}{t_{2}} \wedge \frac{t_{2}}{u_{2}}\right)
$$

which together with Lemma 3.6 shows that

$$
\tilde{K}\left(y_{1}, y_{2}, x_{3}\right)=\int_{\mathbb{R}} K^{\sharp}\left(y_{1}, y_{2}, x_{3}-y_{3}\right)\left(\psi_{u_{2}}^{(2)} * \psi_{t_{2}}^{(2)}\right)\left(y_{3}\right) d y_{3}
$$


satisfies all the conditions of Lemma 3.3 for any fixed $x_{3} \in \mathbb{R}$ with

$$
C_{\tilde{K}} \leq C\left(\frac{u_{2}}{t_{2}} \wedge \frac{t_{2}}{u_{2}}\right) \frac{u_{2} \vee t_{2}}{\left(u_{2} \vee t_{2}+\left|x_{3}\right|\right)^{2}}
$$

From this, Lemma 3.5, and Lemma 3.3, it follows that

$$
\begin{aligned}
& \left|\left(\psi_{u_{1} u_{2}} * K * \psi_{t_{1} t_{2}}\right)\left(x_{1}, x_{2}\right)\right| \\
& \leq C\left(\frac{u_{1}}{t_{1}} \wedge \frac{t_{1}}{u_{1}}\right)\left(\frac{u_{2}}{t_{2}} \wedge \frac{t_{2}}{u_{2}}\right) \int_{\mathbb{R}} \frac{u_{1} \vee t_{1}}{\left(u_{1} \vee t_{1}+\left\|\left(x_{1}, x_{2}-x_{3}\right)\right\|\right)^{4}} \frac{u_{2} \vee t_{2}}{\left(u_{2} \vee t_{2}+\left|x_{3}\right|\right)^{2}} d x_{3},
\end{aligned}
$$

which together with the estimate (11) yields that

$$
\begin{aligned}
\mid\left(\psi_{u_{1} u_{2}} * K\right. & \left.* \psi_{t_{1} t_{2}}\right)\left(x_{1}, x_{2}\right) \mid \\
\leq C & \left(\frac{u_{1}}{t_{1}} \wedge \frac{t_{1}}{u_{1}}\right)\left(\frac{u_{2}}{t_{2}} \wedge \frac{t_{2}}{u_{2}}\right) \\
& \times\left\{\frac{u_{1} \vee t_{1}}{\left(u_{1} \vee t_{1}+\left\|\left(x_{1}, x_{2}\right)\right\|\right)^{4}}+\frac{u_{1} \vee t_{1}}{\left(u_{1} \vee t_{1}+\left|x_{1}\right|\right)^{2}} \frac{u_{2} \vee t_{2}}{\left(u_{2} \vee t_{2}+\left|x_{2}\right|\right)^{2}}\right\} .
\end{aligned}
$$

Inserting the last estimate into (23) yields that

$$
\begin{aligned}
\left|\left(\psi_{u_{1} u_{2}} * K * f\right)\left(x_{1}, x_{2}\right)\right| & \leq \int_{0}^{\infty} \int_{0}^{\infty}\left(\frac{u_{1}}{t_{1}} \wedge \frac{t_{1}}{u_{1}}\right)\left(\frac{u_{2}}{t_{2}} \wedge \frac{t_{2}}{u_{2}}\right) \\
& \times\left\{M\left(\psi_{t_{1} t_{2}} * f\right)\left(x_{1}, x_{2}\right)+M_{s}\left(\psi_{t_{1} t_{2}} * f\right)\left(x_{1}, x_{2}\right)\right\} \frac{d t_{1}}{t_{1}} \frac{d t_{2}}{t_{2}} .
\end{aligned}
$$

Replacing (12) with (24) in the proof of Theorem 2.6 and repeating the proof there, we complete the proof of Theorem 3.1 and we omit the details.

\section{Lifting properties}

In this section, we use Theorem 3.1 in the last section to establish the lifting property of $\dot{B}_{p q}^{s}\left(\mathbb{R}^{2}\right)$ and $\dot{F}_{p q}^{s}\left(\mathbb{R}^{2}\right)$. First, we introduce the following Riesz potential operators related to flag singular integrals.

Definition 4.1. Let $\left\{\psi_{l_{1} l_{2}}\right\}_{l_{1}, l_{2} \in \mathbb{Z}}$ be the same as in Lemma 2.4 and $\left(\alpha_{1}, \alpha_{2}\right) \in \mathbb{R} \times \mathbb{R}$. Then the Riesz potential operator $I_{\left(\alpha_{1}, \alpha_{2}\right)}$ for $f \in \mathcal{S}_{\infty, F}\left(\mathbb{R}^{2}\right)^{\prime}$ is defined by

$$
I_{\left(\alpha_{1}, \alpha_{2}\right)}(f)\left(x_{1}, x_{2}\right)=\sum_{l_{1}=-\infty}^{\infty} \sum_{l_{2}=-\infty}^{\infty} 2^{-l_{1} \alpha_{1}} 2^{-l_{2} \alpha_{2}}\left(\psi_{l_{1} l_{2}} * f\right)\left(x_{1}, x_{2}\right),
$$

where $\left(x_{1}, x_{2}\right) \in \mathbb{R}^{2}$. 
From Definition 4.1, it is easy to deduce the following simple property of Riesz potential operators.

Proposition 4.2. Let $\left\{\psi_{l_{1}}^{(1)}\right\}_{l_{1} \in \mathbb{Z}}$ and $\left\{\psi_{l_{2}}^{(2)}\right\}_{l_{2} \in \mathbb{Z}}$ be the same as in Lemma 2.4. For $f \in \mathcal{S}\left(\mathbb{R}^{2}\right)^{\prime}$ and $\left(x_{1}, x_{2}\right) \in \mathbb{R}^{2}$, let

$$
I_{\alpha_{1}}^{(1)}(f)\left(x_{1}, x_{2}\right)=\sum_{l_{1}=-\infty}^{\infty} 2^{-l_{1} \alpha_{1}}\left(\psi_{l_{1}}^{(1)} * f\right)\left(x_{1}, x_{2}\right),
$$

and for $f \in \mathcal{S}(\mathbb{R})^{\prime}$ and $x_{3} \in \mathbb{R}$, let

$$
I_{\alpha_{2}}^{(2)}(f)\left(x_{3}\right)=\sum_{l_{2}=-\infty}^{\infty} 2^{-l_{2} \alpha_{2}}\left(\psi_{l_{2}}^{(2)} * f\right)\left(x_{3}\right) .
$$

Then

$$
I_{\left(\alpha_{1}, \alpha_{2}\right)}=I_{\alpha_{1}}^{(1)} I_{\alpha_{2}}^{(2)}=I_{\alpha_{2}}^{(2)} I_{\alpha_{1}}^{(1)} .
$$

One of the main theorems in this section is the following boundedness of $I_{\left(\alpha_{1}, \alpha_{2}\right)}$ on $\dot{B}_{p q}^{s}\left(\mathbb{R}^{2}\right)$ and $\dot{F}_{p q}^{s}\left(\mathbb{R}^{2}\right)$ as below.

Proposition 4.3. Let $\left|s_{i}\right|<1,\left|\alpha_{i}\right|<1,\left|s_{i}+\alpha_{i}\right|<1$ for $i=1,2, s=\left(s_{1}, s_{2}\right)$ and $s+\alpha=\left(s_{1}+\alpha_{1}, s_{2}+\alpha_{2}\right)$. Then

(i) If $p, q \in[1, \infty], I_{\left(\alpha_{1}, \alpha_{2}\right)}$ is bounded from $\dot{B}_{p q}^{s}\left(\mathbb{R}^{2}\right)$ to $\dot{B}_{p q}^{s+\alpha}\left(\mathbb{R}^{2}\right)$, namely, there exists a positive constant $C$ such that for all $f \in \dot{B}_{p q}^{s}\left(\mathbb{R}^{2}\right)$,

$$
\left\|I_{\left(\alpha_{1}, \alpha_{2}\right)}(f)\right\|_{\dot{B}_{p q}^{s+\alpha}\left(\mathbb{R}^{2}\right)} \leq C\|f\|_{\dot{B}_{p q}^{s}\left(\mathbb{R}^{2}\right)} .
$$

(ii) If $p \in(1, \infty)$ and $q \in(1, \infty], I_{\left(\alpha_{1}, \alpha_{2}\right)}$ is bounded from $\dot{F}_{p q}^{s}\left(\mathbb{R}^{2}\right)$ to $\dot{F}_{p q}^{s+\alpha}\left(\mathbb{R}^{2}\right)$, namely, there exists a positive constant $C$ such that for all $f \in \dot{F}_{p q}^{s}\left(\mathbb{R}^{2}\right)$,

$$
\left\|I_{\left(\alpha_{1}, \alpha_{2}\right)}(f)\right\|_{\dot{F}_{p q}^{s+\alpha}\left(\mathbb{R}^{2}\right)} \leq C\|f\|_{\dot{F}_{p q}^{s}\left(\mathbb{R}^{2}\right)} .
$$

To prove Proposition 4.3, we first establish the following basic estimate. In what follows, for any $a, b \in \mathbb{R}$, let $a \wedge b=\min \{a, b\}$.

Lemma 4.4. For $i=1$, 2, let $I_{\alpha_{i}}^{(i)}$ be the same as in Proposition 4.2 and $\left\{\tilde{\psi}_{k_{i}}^{(i)}\right\}_{k_{i} \in \mathbb{Z}}$ be the same as in Lemma 2.4 with $\operatorname{supp} \tilde{\psi}^{(1)} \subset\left\{\left(x_{1}, x_{2}\right) \in \mathbb{R}^{2}:\left\|\left(x_{1}, x_{2}\right)\right\| \leq 1\right\}$ and

$$
\operatorname{supp} \tilde{\psi}^{(2)} \subset\left\{x_{3} \in \mathbb{R}:\left|x_{3}\right| \leq 1\right\} .
$$

Then, 
(i) For $\left|\alpha_{1}\right|<1$ and any $\epsilon_{1}>0$, there exists a positive constant $C_{\epsilon_{1}, \alpha_{1}}$ such that for all $k_{1}, j_{1} \in \mathbb{Z}$ and all $\left(x_{1}, x_{2}\right) \in \mathbb{R}^{2}$,

$$
\begin{aligned}
\mid \tilde{\psi}_{k_{1}}^{(1)} * I_{\alpha_{1}}^{(1)} * & \tilde{\psi}_{j_{1}}^{(1)}\left(x_{1}, x_{2}\right) \mid \\
& \leq C_{\epsilon_{1}, \alpha_{1}}\left(1+\left|k_{1}-j_{1}\right|\right) 2^{-\left(k_{1} \wedge j_{1}\right) \alpha_{1}} 2^{-\left|k_{1}-j_{1}\right|\left(1+\alpha_{1} \wedge 0\right)} \\
& \times \frac{2^{-\left(k_{1} \wedge j_{1}\right)\left(1-\alpha_{1}-\epsilon_{1}\right)}}{\left(2^{-\left(k_{1} \wedge j_{1}\right)}+\left\|\left(x_{1}, x_{2}\right)\right\|\right)^{4-\alpha_{1}-\epsilon_{1}}} .
\end{aligned}
$$

(ii) For $\left|\alpha_{2}\right|<1$ and any $\epsilon_{2}>0$, there exists a positive constant $C_{\epsilon_{2}, \alpha_{2}}$ such that for all $k_{2}, j_{2} \in \mathbb{Z}$ and all $x_{3} \in \mathbb{R}$,

$$
\begin{aligned}
\left|\tilde{\psi}_{k_{2}}^{(2)} * I_{\alpha_{2}}^{(2)} * \tilde{\psi}_{j_{2}}^{(2)}\left(x_{3}\right)\right| & \\
\leq C_{\epsilon_{2}, \alpha_{2}}\left(1+\left|k_{2}-j_{2}\right|\right) 2^{-\left(k_{2} \wedge j_{2}\right) \alpha_{2}} & 2^{-\left|k_{2}-j_{2}\right|\left(1+\alpha_{2} \wedge 0\right)} \\
& \times \frac{2^{-\left(k_{2} \wedge j_{2}\right)\left(1-\alpha_{2}-\epsilon_{2}\right)}}{\left(2^{-\left(k_{2} \wedge j_{2}\right)}+\left|x_{3}\right|\right)^{2-\alpha_{2}-\epsilon_{2}}}
\end{aligned}
$$

Proof. By similarity, we only show (i). Without loss of generality, we may further assume that $j_{1} \geq k_{1}$. In this case, we write

$$
\begin{aligned}
\tilde{\psi}_{k_{1}}^{(1)} * I_{\alpha_{1}}^{(1)} * \tilde{\psi}_{j_{1}}^{(1)} & \left(x_{1}, x_{2}\right) \\
& =\sum_{l_{1}=-\infty}^{k_{1}} 2^{-l_{1} \alpha_{1}} \tilde{\psi}_{k_{1}}^{(1)} * \psi_{l_{1}}^{(1)} * \tilde{\psi}_{j_{1}}^{(1)}\left(x_{1}, x_{2}\right)+\sum_{l_{1}=k_{1}+1}^{j_{1}} \cdots+\sum_{l_{1}=j_{1}+1}^{\infty} \ldots \\
& =O_{1}+O_{2}+O_{3} .
\end{aligned}
$$

We now consider two cases.

- Case 1: $\left\|\left(x_{1}, x_{2}\right)\right\| \geq 5 \cdot 2^{-k_{1}}$. In this case, by the vanishing moment of $\tilde{\psi}^{(1)}$ and the mean value theorem,

$$
\begin{aligned}
\left|O_{1}\right|=\mid & \sum_{l_{1}=-\infty}^{k_{1}} 2^{-l_{1} \alpha_{1}} \int_{\mathbb{R}^{2}} \int_{\mathbb{R}^{2}} \tilde{\psi}_{k_{1}}^{(1)}\left(x_{1}-y_{1}, x_{2}-y_{2}\right) \\
& \times\left[\psi_{l_{1}}^{(1)}\left(y_{1}-u_{1}, y_{2}-u_{2}\right)-\psi_{l_{1}}^{(1)}\left(y_{1}, y_{2}\right)\right] \tilde{\psi}_{j_{1}}^{(1)}\left(u_{1}, u_{2}\right) d y_{1} d y_{2} d u_{1} d u_{2} \mid \leq
\end{aligned}
$$




$$
\begin{aligned}
& \leq \sum_{l_{1}=-\infty}^{k_{1}} 2^{-l_{1} \alpha_{1}} \int_{\mathbb{R}^{2}} \int_{\mathbb{R}^{2}}\left|\tilde{\psi}_{k_{1}}^{(1)}\left(y_{1}, y_{2}\right)\right| \\
& \times\left[\left|\frac{\partial \psi_{l_{1}}^{(1)}}{\partial y_{1}}\left(x_{1}-y_{1}-\theta u_{1}, x_{2}-y_{2}-\theta u_{2}\right)\right|\left|u_{1}\right|\right. \\
& \left.\quad+\left|\frac{\partial \psi_{l_{1}}^{(1)}}{\partial y_{2}}\left(x_{1}-y_{1}-\theta u_{1}, x_{2}-y_{2}-\theta u_{2}\right)\right|\left|u_{2}\right|\right] \\
& \quad \times\left|\tilde{\psi}_{j_{1}}^{(1)}\left(u_{1}, u_{2}\right)\right| d y_{1} d y_{2} d u_{1} d u_{2},
\end{aligned}
$$

where $\theta \in(0,1)$. The support condition of $\tilde{\psi}^{(1)}$ yields that

$$
\begin{aligned}
\left\|\left(x_{1}-y_{1}-\theta u_{1}, x_{2}-y_{2}-\theta u_{2}\right)\right\| & \geq\left\|\left(x_{1}, x_{2}\right)\right\|-\left\|\left(y_{1}, y_{2}\right)\right\|-\left\|\left(u_{1}, u_{2}\right)\right\| \\
& \geq\left\|\left(x_{1}, x_{2}\right)\right\| / 2 .
\end{aligned}
$$

From this, it follows that $\left|O_{1}\right|$ is further controlled by

$$
\left|O_{1}\right| \leq C \sum_{l_{1}=-\infty}^{k_{1}} 2^{l_{1} \epsilon_{1}} 2^{-j_{1}} \frac{1}{\left\|\left(x_{1}, x_{2}\right)\right\|^{4-\alpha_{1}-\epsilon_{1}}} \leq C 2^{-k_{1} \alpha_{1}} 2^{k_{1}-j_{1}} \frac{2^{-k_{1}\left(1-\alpha_{1}-\epsilon_{1}\right)}}{\left\|\left(x_{1}, x_{2}\right)\right\|^{4-\alpha_{1}-\epsilon_{1}}}
$$

Similarly, for $\mathrm{O}_{2}$, we have

$$
\begin{aligned}
\left|O_{2}\right|= & \mid \sum_{l_{1}=k_{1}+1}^{j_{1}} 2^{-l_{1} \alpha_{1}} \int_{\mathbb{R}^{2}} \int_{\mathbb{R}^{2}} \psi_{l_{1}}^{(1)}\left(x_{1}-y_{1}, x_{2}-y_{2}\right) \\
& \quad \times\left[\tilde{\psi}_{k_{1}}^{(1)}\left(y_{1}-u_{1}, y_{2}-u_{2}\right)-\tilde{\psi}_{k_{1}}^{(1)}\left(y_{1}, y_{2}\right)\right] \tilde{\psi}_{j_{1}}^{(1)}\left(u_{1}, u_{2}\right) d y_{1} d y_{2} d u_{1} d u_{2} \mid \\
\leq & C 2^{k_{1}-j_{1}} \frac{1}{\left\|\left(x_{1}, x_{2}\right)\right\|^{4-\alpha_{1}}} \sum_{l_{1}=k_{1}+1}^{j_{1}} 2^{-l_{1}}=C 2^{-k_{1} \alpha_{1}} 2^{k_{1}-j_{1}} \frac{2^{-k_{1}\left(1-\alpha_{1}\right)}}{\left\|\left(x_{1}, x_{2}\right)\right\|^{4-\alpha_{1}}}
\end{aligned}
$$

since

$$
\left\|\left(x_{1}-y_{1}, x_{2}-y_{2}\right)\right\| \geq\left\|\left(x_{1}, x_{2}\right)\right\|-\left\|\left(y_{1}, y_{2}\right)\right\| \geq\left\|\left(x_{1}, x_{2}\right)\right\| / 2 \text {. }
$$


For $O_{3}$, by the vanishing moment of $\psi^{(1)}$ and the mean value theorem, we have

$$
\begin{aligned}
\left|O_{3}\right|=\mid & \sum_{l_{1}=j_{1}+1}^{\infty} 2^{-l_{1} \alpha_{1}} \int_{\mathbb{R}^{2}} \int_{\mathbb{R}^{2}}\left[\tilde{\psi}_{k_{1}}^{(1)}\left(u_{1}-y_{1}, u_{2}-y_{2}\right)-\tilde{\psi}_{k_{1}}^{(1)}\left(u_{1}, u_{2}\right)\right] \\
& \times \psi_{l_{1}}^{(1)}\left(y_{1}, y_{2}\right) \tilde{\psi}_{j_{1}}^{(1)}\left(x_{1}-u_{1}, x_{2}-u_{2}\right) d u_{1} d u_{2} d y_{1} d y_{2} \mid \\
\leq & \sum_{l_{1}=j_{1}+1}^{\infty} 2^{-l_{1} \alpha_{1}} \int_{\mathbb{R}^{2}} \int_{\left\|\left(u_{1}, u_{2}\right)\right\| \leq 2^{-k_{1}}}\left|\tilde{\psi}_{k_{1}}^{(1)}\left(u_{1}-y_{1}, u_{2}-y_{2}\right)-\tilde{\psi}_{k_{1}}^{(1)}\left(u_{1}, u_{2}\right)\right| \\
& \times\left|\psi_{l_{1}}^{(1)}\left(y_{1}, y_{2}\right) \tilde{\psi}_{j_{1}}^{(1)}\left(x_{1}-u_{1}, x_{2}-u_{2}\right)\right| d u_{1} d u_{2} d y_{1} d y_{2}+\sum_{l_{1}=j_{1}+1}^{\infty} 2^{-l_{1} \alpha_{1}} \\
& \times \int_{\mathbb{R}^{2}} \int_{\left\|\left(u_{1}-y_{1}, u_{2}-y_{2}\right)\right\| \leq 2^{-k_{1}}}\left|\tilde{\psi}_{k_{1}}^{(1)}\left(u_{1}-y_{1}, u_{2}-y_{2}\right)-\tilde{\psi}_{k_{1}}^{(1)}\left(u_{1}, u_{2}\right)\right| \\
& \times\left|\psi_{l_{1}}^{(1)}\left(y_{1}, y_{2}\right) \tilde{\psi}_{j_{1}}^{(1)}\left(x_{1}-u_{1}, x_{2}-u_{2}\right)\right| d u_{1} d u_{2} d y_{1} d y_{2}=O_{3}^{1}+O_{3}^{2} .
\end{aligned}
$$

For $O_{3}^{1}$, we have

$$
\left\|\left(x_{1}-u_{1}, x_{2}-u_{2}\right)\right\| \geq\left\|\left(x_{1}, x_{2}\right)\right\|-\left\|\left(u_{1}, u_{2}\right)\right\| \geq\left\|\left(x_{1}, x_{2}\right)\right\| / 2 ;
$$

and, for $O_{3}^{2}$, we have

$$
\left\|\left(y_{1}, y_{2}\right)\right\| \geq\left\|\left(x_{1}, x_{2}\right)\right\|-\left\|\left(y_{1}-u_{1}, y_{2}-u_{2}\right)\right\|-\left\|\left(u_{1}-x_{1}, u_{2}-x_{2}\right)\right\| \geq\left\|\left(x_{1}, x_{2}\right)\right\| / 2 \text {. }
$$

These facts, respectively, imply that

and

$$
\begin{aligned}
O_{3}^{1} & \leq C \frac{1}{\left\|\left(x_{1}, x_{2}\right)\right\|^{4-\alpha_{1}}} 2^{k_{1}-j_{1}\left(1-\alpha_{1}\right)} \sum_{l_{1}=j_{1}+1}^{\infty} 2^{-l_{1}\left(1+\alpha_{1}\right)} \\
& \leq C 2^{-k_{1} \alpha_{1}} 2^{2\left(k_{1}-j_{1}\right)} \frac{2^{-k_{1}\left(1-\alpha_{1}\right)}}{\left\|\left(x_{1}, x_{2}\right)\right\|^{4-\alpha_{1}}}
\end{aligned}
$$

$$
\begin{aligned}
O_{3}^{2} & \leq C \frac{1}{\left\|\left(x_{1}, x_{2}\right)\right\|^{4-\alpha_{1}}} 2^{k_{1}} \sum_{l_{1}=j_{1}+1}^{\infty} 2^{-2 l_{1}} \\
& \leq C 2^{-k_{1} \alpha_{1}} 2^{2\left(k_{1}-j_{1}\right)} \frac{2^{-k_{1}\left(1-\alpha_{1}\right)}}{\left\|\left(x_{1}, x_{2}\right)\right\|^{4-\alpha_{1}}} .
\end{aligned}
$$

This finishes the proof of case 1 .

- Case 2: $\left\|\left(x_{1}, x_{2}\right)\right\|<5 \cdot 2^{-k_{1}}$. In this case, by (25), we have

$$
\left|O_{1}\right| \leq C \sum_{l_{1}=-\infty}^{k_{1}} 2^{4 l_{1}-l_{1} \alpha_{1}-j_{1}}=C 2^{-k_{1} \alpha_{1}} 2^{k_{1}-j_{1}} 2^{3 k_{1}}
$$


by (26), we obtain

$$
\left|O_{2}\right| \leq C \sum_{l_{1}=k_{1}+1}^{j_{1}} 2^{4 k_{1}-l_{1} \alpha_{1}-j_{1}}= \begin{cases}\left(j_{1}-k_{1}\right) 2^{k_{1}-j_{1}} 2^{3 k_{1}}, & \alpha_{1}=0 \\ C 2^{-k_{1} \alpha_{1}} 2^{k_{1}-j_{1}} 2^{3 k_{1}}, & \alpha_{1}>0 \\ C 2^{-k_{1} \alpha_{1}} 2^{\left(k_{1}-j_{1}\right)\left(1+\alpha_{1}\right)} 2^{3 k_{1}}, & \alpha_{1}<0\end{cases}
$$

and, finally by (27), we have

$$
\left|O_{3}\right| \leq C \sum_{l_{1}=j_{1}+1}^{\infty} 2^{4 k_{1}-l_{1} \alpha_{1}-l_{1}}=C 2^{-k_{1} \alpha_{1}} 2^{\left(k_{1}-j_{1}\right)\left(1+\alpha_{1}\right)} 2^{3 k_{1}},
$$

which are desired estimates. This finishes the proof of case 2 and, hence, the proof of Lemma 4.4

Proof of Proposition 4.3. Let $\left\{\tilde{\psi}_{k_{i}}^{(i)}\right\}_{k_{i} \in \mathbb{Z}}$ with $i=1,2$ and $\left\{\psi_{l_{1} l_{2}}\right\}_{l_{1}, l_{2} \in \mathbb{Z}}$ be the same, respectively, as in Lemma 4.4 and Lemma 2.4. By Theorem 2.8 and Lemma 2.4, we have

$$
\begin{aligned}
\left\|I_{\left(\alpha_{1}, \alpha_{2}\right)}(f)\right\|_{\dot{B}_{p q}^{s+\alpha}\left(\mathbb{R}^{2}\right)} & \\
\leq C & \left\{\sum_{k_{1}=-\infty}^{\infty} \sum_{k_{2}=-\infty}^{\infty} 2^{k_{1}\left(s_{1}+\alpha_{1}\right) q} 2^{k_{2}\left(s_{2}+\alpha_{2}\right) q}\right. \\
\quad & \left.\times\left\|\sum_{j_{1}=-\infty}^{\infty} \sum_{j_{2}=-\infty}^{\infty} \tilde{\psi}_{k_{1} k_{2}} * I_{\left(\alpha_{1}, \alpha_{2}\right)} * \tilde{\psi}_{j_{1} j_{2}} * \tilde{\psi}_{j_{1} j_{2}} * f\right\|_{L^{p}\left(\mathbb{R}^{2}\right)}^{q}\right\}^{1 / q} .
\end{aligned}
$$

Lemma 4.4 further yields that for all $\left(x_{1}, x_{2}\right) \in \mathbb{R}^{2}$,

$$
\begin{aligned}
\mid \tilde{\psi}_{k_{1} k_{2}} * I_{\left(\alpha_{1}, \alpha_{2}\right)} * & \tilde{\psi}_{j_{1} j_{2}}\left(x_{1}, x_{2}\right) \mid \\
\leq C & 2^{-\left(k_{1} \wedge j_{1}\right) \alpha_{1}-\left|k_{1}-j_{1}\right|\left(1+\alpha_{1} \wedge 0\right)-\left(k_{2} \wedge j_{2}\right) \alpha_{2}-\left|k_{2}-j_{2}\right|\left(1+\alpha_{2} \wedge 0\right)} \\
\times & \left(1+\left|k_{1}-j_{1}\right|\right)\left(1+\left|k_{2}-j_{2}\right|\right) \\
& \times\left\{\frac{2^{-\left(k_{1} \wedge j_{1}\right)\left(1-\alpha_{1}-\epsilon_{1}\right)}}{\left(2^{-\left(k_{1} \wedge j_{1}\right)}+\left\|\left(x_{1}, x_{2}\right)\right\|\right)^{4-\alpha_{1}-\epsilon_{1}}}\right. \\
& \left.+\frac{2^{-\left(k_{1} \wedge j_{1}\right)\left(1-\alpha_{1}-\epsilon_{1}\right)}}{\left(2^{-\left(k_{1} \wedge j_{1}\right)}+\left|x_{1}\right|\right)^{2-\alpha_{1}-\epsilon_{1}}} \frac{2^{-\left(k_{2} \wedge j_{2}\right)\left(1-\alpha_{2}-\epsilon_{2}\right)}}{\left(2^{-\left(k_{2} \wedge j_{2}\right)}+\left|x_{2}\right|\right)^{2-\alpha_{2}-\epsilon_{2}}}\right\} .
\end{aligned}
$$

From this, the Minkowski inequality, and the boundedness of $M_{s}$ in $L^{p}\left(\mathbb{R}^{2}\right)$, it follows 
that, for $p \in(1, \infty]$,

$$
\begin{aligned}
&\left\|I_{\left(\alpha_{1}, \alpha_{2}\right)}(f)\right\|_{\dot{B}_{p q}^{s+\alpha}\left(\mathbb{R}^{2}\right)} \\
& \leq C\left\{\sum_{k_{1}=-\infty}^{\infty} \sum_{k_{2}=-\infty}^{\infty} 2^{k_{1}\left(s_{1}+\alpha_{1}\right) q} 2^{k_{2}\left(s_{2}+\alpha_{2}\right) q}\right. \\
& \quad \times\left[\sum_{j_{1}=-\infty}^{\infty} \sum_{j_{2}=-\infty}^{\infty} 2^{-\left(k_{1} \wedge j_{1}\right) \alpha_{1}-\left|k_{1}-j_{1}\right|\left(1+\alpha_{1} \wedge 0\right)-\left(k_{2} \wedge j_{2}\right) \alpha_{2}-\left|k_{2}-j_{2}\right|\left(1+\alpha_{2} \wedge 0\right)}\right. \\
&\left.\left.\quad \times\left(1+\left|k_{1}-j_{1}\right|\right)\left(1+\left|k_{2}-j_{2}\right|\right)\left\|\tilde{\psi}_{j_{1} j_{2}} * f\right\|_{L^{p}\left(\mathbb{R}^{2}\right)}\right]^{q}\right\}^{1 / q}
\end{aligned}
$$

If $p=1$, by the Minkowski inequality and the Fubini theorem, we also obtain the same estimate. Now the assumptions that $\left|s_{i}\right|<1$ and $\left|s_{i}+\alpha_{i}\right|<1$ imply that

$$
\sup _{k_{i} \in \mathbb{Z}} \sum_{j_{i}=-\infty}^{\infty}\left(1+\left|k_{i}-j_{i}\right|\right) 2^{-\left(k_{i} \wedge j_{i}\right) \alpha_{i}-\left|k_{i}-j_{i}\right|\left(1+\alpha_{i} \wedge 0\right)+\left(k_{i}-j_{i}\right) s_{i}+k_{i} \alpha_{i}}<\infty
$$

and

$$
\sup _{j_{i} \in \mathbb{Z}} \sum_{k_{i}=-\infty}^{\infty}\left(1+\left|k_{i}-j_{i}\right|\right) 2^{-\left(k_{i} \wedge j_{i}\right) \alpha_{i}-\left|k_{i}-j_{i}\right|\left(1+\alpha_{i} \wedge 0\right)+\left(k_{i}-j_{i}\right) s_{i}+k_{i} \alpha_{i}}<\infty,
$$

where $i=1,2$. Combining these estimates (30) and (31) with (29) and using the Hölder inequality yield that

$$
\begin{aligned}
\left\|I_{\left(\alpha_{1}, \alpha_{2}\right)}(f)\right\|_{\dot{B}_{p q}^{s+\alpha}\left(\mathbb{R}^{2}\right)} & \leq C\left\{\sum_{j_{1}=-\infty}^{\infty} \sum_{j_{2}=-\infty}^{\infty} 2^{j_{1} s_{1} q} 2^{j_{2} s_{2} q}\left\|\tilde{\psi}_{j_{1} j_{2}} * f\right\|_{L^{p}\left(\mathbb{R}^{2}\right)}^{q}\right\}^{1 / q} \\
& \leq C\|f\|_{\dot{B}_{p q}^{s}\left(\mathbb{R}^{2}\right)}
\end{aligned}
$$

which completes the proof of Proposition 4.3 (i).

To prove Proposition 4.3 (ii), by Theorem 2.8, Lemma 2.4, the estimates (28), (30), and (31), and the Hölder inequality, we obtain

$$
\begin{aligned}
& \left\|I_{\left(\alpha_{1}, \alpha_{2}\right)}(f)\right\|_{\dot{F}_{p q}^{s+\alpha}\left(\mathbb{R}^{2}\right)} \\
& \quad \leq C\left\|\left\{\sum_{k_{1}=-\infty}^{\infty} \sum_{k_{2}=-\infty}^{\infty} 2^{k_{1}\left(s_{1}+\alpha_{1}\right) q} 2^{k_{2}\left(s_{2}+\alpha_{2}\right) q}\left|\tilde{\psi}_{k_{1} k_{2}} * I_{\left(\alpha_{1}, \alpha_{2}\right)}(f)\right|^{q}\right\}^{1 / q}\right\|_{L^{p}\left(\mathbb{R}^{2}\right)} \leq
\end{aligned}
$$




$$
\begin{aligned}
& \leq C \|\left\{\sum_{k_{1}=-\infty}^{\infty} \sum_{k_{2}=-\infty}^{\infty} 2^{k_{1}\left(s_{1}+\alpha_{1}\right) q} 2^{k_{2}\left(s_{2}+\alpha_{2}\right) q}\right. \\
& \times\left[\sum_{j_{1}=-\infty}^{\infty} \sum_{j_{2}=-\infty}^{\infty} 2^{-\left(k_{1} \wedge j_{1}\right) \alpha_{1}-\left|k_{1}-j_{1}\right|\left(1+\alpha_{1} \wedge 0\right)-\left(k_{2} \wedge j_{2}\right) \alpha_{2}-\left|k_{2}-j_{2}\right|\left(1+\alpha_{2} \wedge 0\right)}\right. \\
& \left.\left.\times\left(1+\left|k_{1}-j_{1}\right|\right)\left(1+\left|k_{2}-j_{2}\right|\right) M_{s}\left(\tilde{\psi}_{j_{1} j_{2}} * f\right)\right]^{q}\right\}^{1 / q}\left\|_{L^{p}\left(\mathbb{R}^{2}\right)} \leq C\right\| f \|_{\dot{F}_{p q}^{s}\left(\mathbb{R}^{2}\right)} \\
& \leq C\left\|\left\{\sum_{j_{1}=-\infty}^{\infty} \sum_{j_{2}=-\infty}^{\infty} 2^{j_{1} s_{1} q_{2} 2^{j_{2} s_{2} q}\left|\tilde{\psi}_{j_{1} j_{2}} * f\right|^{q}}\right\}^{1 / q}\right\| \|_{L^{p}\left(\mathbb{R}^{2}\right)} \leq C
\end{aligned}
$$

where, in the second-to-last inequality, we used the vector-valued inequality of Fefferman-Stein in [1]. This finishes the proof of Proposition 4.3.

We now establish the converse of Proposition 4.3.

Proposition 4.5. Let $\left|s_{i}\right|<1,\left|s_{i}+\alpha_{i}\right|<1$ for $i=1,2, s=\left(s_{1}, s_{2}\right)$, and $s+\alpha=$ $\left(s_{1}+\alpha_{1}, s_{2}+\alpha_{2}\right)$. Then there exist a positive constant $C$ and $\alpha_{i}^{0}\left(s_{1}, s_{2}\right) \in(0,1)$ such that, if $\left|\alpha_{i}\right|<\alpha_{i}^{0}\left(s_{1}, s_{2}\right)$ with $i=1,2$,

$$
\|f\|_{\dot{B}_{p q}^{s}\left(\mathbb{R}^{2}\right)} \leq C\left\|I_{\left(\alpha_{1}, \alpha_{2}\right)}(f)\right\|_{\dot{B}_{p q}^{s+\alpha}\left(\mathbb{R}^{2}\right)}
$$

for all $f \in \dot{B}_{p q}^{s}\left(\mathbb{R}^{2}\right)$ with $p, q \in[1, \infty]$, and for all $f \in \dot{F}_{p q}^{s}\left(\mathbb{R}^{2}\right)$ with $p \in(1, \infty)$ and $q \in(1, \infty]$,

$$
\|f\|_{\dot{F}_{p q}^{s}\left(\mathbb{R}^{2}\right)} \leq C\left\|I_{\left(\alpha_{1}, \alpha_{2}\right)}(f)\right\|_{\dot{F}_{p q}^{s+\alpha}\left(\mathbb{R}^{2}\right)} .
$$

Proof. The key of the proof is to show that the operator $I_{\left(-\alpha_{1},-\alpha_{2}\right)} I_{\left(\alpha_{1}, \alpha_{2}\right)}$ is invertible in $\dot{B}_{p q}^{s}\left(\mathbb{R}^{2}\right)$ and $\dot{F}_{p q}^{s}\left(\mathbb{R}^{2}\right)$ if $\alpha_{1}$ and $\alpha_{2}$ are small. To this end, we need to prove that the operator $I-I_{\left(-\alpha_{1},-\alpha_{2}\right)} I_{\left(\alpha_{1}, \alpha_{2}\right)}$ is bounded on $\dot{B}_{p q}^{s}\left(\mathbb{R}^{2}\right)$ and $\dot{F}_{p q}^{s}\left(\mathbb{R}^{2}\right)$ with operator norms less than 1 when $\alpha_{1}$ and $\alpha_{2}$ are small, where $I$ is the identity operator. We obtain this by using Theorem 3.1. Let $\left\{\psi_{k_{1} k_{2}}\right\}_{k_{1}, k_{2} \in \mathbb{Z}}$ be the same as in Lemma 2.4. We write

$$
I_{\left(-\alpha_{1},-\alpha_{2}\right)} I_{\left(\alpha_{1}, \alpha_{2}\right)}=\sum_{j_{1}=-\infty}^{\infty} \sum_{j_{2}=-\infty}^{\infty} \sum_{k_{1}=-\infty}^{\infty} \sum_{k_{2}=-\infty}^{\infty} 2^{-k_{1} \alpha_{1}} 2^{-k_{2} \alpha_{2}} \psi_{j_{1} j_{2}} * \psi_{k_{1}+j_{1}, k_{2}+j_{2}}
$$

and

$$
\begin{array}{r}
I-I_{\left(-\alpha_{1},-\alpha_{2}\right)} I_{\left(\alpha_{1}, \alpha_{2}\right)} \\
=\sum_{j_{1}=-\infty}^{\infty} \sum_{j_{2}=-\infty}^{\infty} \sum_{k_{1}=-\infty}^{\infty} \sum_{k_{2}=-\infty}^{\infty}\left(1-2^{-k_{1} \alpha_{1}} 2^{-k_{2} \alpha_{2}}\right) \psi_{j_{1} j_{2}} * \psi_{k_{1}+j_{1}, k_{2}+j_{2}} .
\end{array}
$$


We denote the kernel of $I-I_{\left(-\alpha_{1},-\alpha_{2}\right)} I_{\left(\alpha_{1}, \alpha_{2}\right)}$ simply by $K_{\left(\alpha_{1}, \alpha_{2}\right)}$. Noticing that, for $\left(x_{1}, x_{2}\right) \in \mathbb{R}^{2}$,

$$
\psi_{j_{1} j_{2}} * \psi_{k_{1}+j_{1}, k_{2}+j_{2}}\left(x_{1}, x_{2}\right)=\left(\psi_{j_{1}}^{(1)} * \psi_{k_{1}+j_{1}}^{(1)}\right) *_{2}\left(\psi_{j_{2}}^{(2)} * \psi_{k_{2}+j_{2}}^{(2)}\right)\left(x_{1}, x_{2}\right),
$$

we then have that

$$
\begin{aligned}
K_{\left(\alpha_{1}, \alpha_{2}\right)}^{\sharp}\left(x_{1}, x_{2}, x_{3}\right)= & \sum_{j_{1}=-\infty}^{\infty} \sum_{j_{2}=-\infty}^{\infty} \sum_{k_{1}=-\infty}^{\infty} \sum_{k_{2}=-\infty}^{\infty}\left(1-2^{-k_{1} \alpha_{1}} 2^{-k_{2} \alpha_{2}}\right) \\
& \times\left(\psi_{j_{1}}^{(1)} * \psi_{k_{1}+j_{1}}^{(1)}\right)\left(x_{1}, x_{2}\right)\left(\psi_{j_{2}}^{(2)} * \psi_{k_{2}+j_{2}}^{(2)}\right)\left(x_{3}\right),
\end{aligned}
$$

where $\left(x_{1}, x_{2}, x_{3}\right) \in \mathbb{R}^{2} \times \mathbb{R}$, is the corresponding product kernel on $\mathbb{R}^{2} \times \mathbb{R}$ of $K_{\left(\alpha_{1}, \alpha_{2}\right)}$. We only need to show that $K_{\left(\alpha_{1}, \alpha_{2}\right)}^{\sharp}$ satisfies the conditions of Definition 1.1 with a constant no more than

$$
C \sum_{k_{1}=-\infty}^{\infty} \sum_{k_{2}=-\infty}^{\infty}\left|1-2^{-k_{1} \alpha_{1}} 2^{-k_{2} \alpha_{2}}\right| 2^{-\left|k_{1}\right|} 2^{-\left|k_{2}\right|},
$$

where $C$ is a positive constant independent of $\alpha_{1}$ and $\alpha_{2}$. First, we point out that by a modified argument of (7), we can easily to show that for $\gamma_{1}, \gamma_{2}, \gamma_{3} \in \mathbb{Z}_{+}$, $\left(x_{1}, x_{2}\right) \in \mathbb{R}^{2}$, and $x_{3} \in \mathbb{R}$,

$$
\begin{aligned}
& \left|\partial_{x_{1}}^{\gamma_{1}} \partial_{x_{2}}^{\gamma_{2}}\left(\psi_{j_{1}}^{(1)} * \psi_{k_{1}+j_{1}}^{(1)}\right)\left(x_{1}, x_{2}\right)\right| \\
& \quad \leq C_{\gamma_{1}, \gamma_{2}} 2^{-\left|k_{1}\right|} \frac{2^{-j_{1} \wedge\left(k_{1}+j_{1}\right)}}{\left(2^{-j_{1} \wedge\left(k_{1}+j_{1}\right)}+\left\|\left(x_{1}, x_{2}\right)\right\|\right)^{4+\gamma_{1}+2 \gamma_{2}}}
\end{aligned}
$$

and

$$
\left|\partial_{x_{3}}^{\gamma_{3}}\left(\psi_{j_{2}}^{(2)} * \psi_{k_{2}+j_{2}}^{(2)}\right)\left(x_{3}\right)\right| \leq C_{\gamma_{3}} 2^{-\left|k_{2}\right|} \frac{2^{-j_{2} \wedge\left(k_{2}+j_{2}\right)}}{\left(2^{-j_{2} \wedge\left(k_{2}+j_{2}\right)}+\left|x_{3}\right|\right)^{2+\gamma_{3}}},
$$

by noticing that $\psi^{(i)}$ for $i=1,2$ are the Schwartz functions. In fact, we only need that these estimates are true for $\gamma_{1}=\gamma_{2}=\gamma_{3}=1$. The estimates (32) and (33) imply that for $\gamma_{1}, \gamma_{2}, \gamma_{3} \in \mathbb{Z}_{+},\left(x_{1}, x_{2}\right) \in \mathbb{R}^{2}$, and $x_{3} \in \mathbb{R}$,

$$
\begin{aligned}
\left|\partial_{x_{1}}^{\gamma_{1}} \partial_{x_{2}}^{\gamma_{2}} \partial_{x_{3}}^{\gamma_{3}} K_{\left(\alpha_{1}, \alpha_{2}\right)}^{\sharp}\left(x_{1}, x_{2}, x_{3}\right)\right| & \\
\leq & \sum_{j_{1}=-\infty}^{\infty} \sum_{j_{2}=-\infty}^{\infty} \sum_{k_{1}=-\infty}^{\infty} \sum_{k_{2}=-\infty}^{\infty}\left|1-2^{-k_{1} \alpha_{1}-k_{2} \alpha_{2}}\right| \\
& \times\left|\partial_{x_{1}}^{\gamma_{1}} \partial_{x_{2}}^{\gamma_{2}}\left(\psi_{j_{1}}^{(1)} * \psi_{k_{1}+j_{1}}^{(1)}\right)\left(x_{1}, x_{2}\right) \partial_{x_{3}}^{\gamma_{3}}\left(\psi_{j_{2}}^{(2)} * \psi_{k_{2}+j_{2}}^{(2)}\right)\left(x_{3}\right)\right| \\
\leq & C\left\{\sum_{k_{1}=-\infty}^{\infty} \sum_{k_{2}=-\infty}^{\infty}\left|1-2^{-k_{1} \alpha_{1}-k_{2} \alpha_{2}}\right| 2^{-\left|k_{1}\right|-\left|k_{2}\right|}\right\} \\
& \times \frac{1}{\left\|\left(x_{1}, x_{2}\right)\right\|^{3+\gamma_{1}+2 \gamma_{2}}} \cdot \frac{1}{\left|x_{3}\right|^{1+\gamma_{3}}} .
\end{aligned}
$$


Let $\varphi$ be a normalized bump function on $\mathbb{R}$ and $\delta>0$. We now estimate, by (32) and (33), that, for $\gamma_{1}, \gamma_{2} \in \mathbb{Z}_{+}$and $\left(x_{1}, x_{2}\right) \in \mathbb{R}^{2}$,

$$
\begin{aligned}
\left|\int_{\mathbb{R}} \partial_{x_{1}}^{\gamma_{1}} \partial_{x_{2}}^{\gamma_{2}} K_{\left(\alpha_{1}, \alpha_{2}\right)}^{\sharp}\left(x_{1}, x_{2}, x_{3}\right) \varphi\left(\delta x_{3}\right) d x_{3}\right| & \\
\leq & \sum_{j_{1}=-\infty}^{\infty} \sum_{j_{2}=-\infty}^{\infty} \sum_{k_{1}=-\infty}^{\infty} \sum_{k_{2}=-\infty}^{\infty}\left|1-2^{-k_{1} \alpha_{1}-k_{2} \alpha_{2}}\right| \\
& \times\left|\int_{\mathbb{R}} \partial_{x_{1}}^{\gamma_{1}} \partial_{x_{2}}^{\gamma_{2}}\left(\psi_{j_{1}}^{(1)} * \psi_{k_{1}+j_{1}}^{(1)}\right)\left(x_{1}, x_{2}\right)\left(\psi_{j_{2}}^{(2)} * \psi_{k_{2}+j_{2}}^{(2)}\right)\left(x_{3}\right) \varphi\left(\delta x_{3}\right) d x_{3}\right| \\
\leq & C \sum_{j_{1}=-\infty}^{\infty} \sum_{j_{2}=-\infty}^{\infty} \sum_{k_{1}=-\infty}^{\infty} \sum_{k_{2}=-\infty}^{\infty}\left|1-2^{-k_{1} \alpha_{1}-k_{2} \alpha_{2}}\right| 2^{-\left|k_{1}\right|} \\
& \times \frac{2^{-j_{1} \wedge\left(k_{1}+j_{1}\right)}}{\left(2^{-j_{1} \wedge\left(k_{1}+j_{1}\right)}+\left\|\left(x_{1}, x_{2}\right)\right\|\right)^{4+\gamma_{1}+2 \gamma_{2}}} \\
& \times\left|\int_{\mathbb{R}}\left(\psi_{j_{2}}^{(2)} * \psi_{k_{2}+j_{2}}^{(2)}\right)\left(x_{3}\right) \varphi\left(\delta x_{3}\right) d x_{3}\right| .
\end{aligned}
$$

We choose $j_{2}^{0} \in \mathbb{Z}$ such that $\delta \leq 2^{-j_{2}^{0} \wedge\left(k_{2}+j_{2}^{0}\right)}<2 \delta$. For this $j_{2}^{0}$ and any $\epsilon>0$, by the vanishing moment of $\psi^{(2)} * \psi^{(2)}$, we have

$$
\begin{aligned}
\sum_{j_{2}=-\infty}^{\infty} \mid \int_{\mathbb{R}}\left(\psi_{j_{2}}^{(2)} * \psi_{k_{2}+j_{2}}^{(2)}\right) & \left(x_{3}\right) \varphi\left(\delta x_{3}\right) d x_{3} \mid \\
\leq & C \sum_{j_{2}=-\infty}^{j_{2}^{0}} 2^{-\left|k_{2}\right|} \int_{\mathbb{R}} \frac{2^{-j_{2} \wedge\left(k_{2}+j_{2}\right)}}{\left(2^{-j_{2} \wedge\left(k_{2}+j_{2}\right)}+\left|x_{3}\right|\right)^{2}}\left|\varphi\left(\delta x_{3}\right)\right| d x_{3} \\
& +\sum_{j_{2}=j_{2}^{0}+1}^{\infty}\left|\int_{\mathbb{R}}\left(\psi_{j_{2}}^{(2)} * \psi_{k_{2}+j_{2}}^{(2)}\right)\left(x_{3}\right)\left[\varphi\left(\delta x_{3}\right)-\varphi(0)\right] d x_{3}\right| \\
\leq & C 2^{-\left|k_{2}\right|}\left\{1+\delta^{\epsilon} \sum_{j_{2}=j_{2}^{0}+1}^{\infty} 2^{-j_{2} \wedge\left(k_{2}+j_{2}\right) \epsilon}\right\} \leq C 2^{-\left|k_{2}\right|},
\end{aligned}
$$

which yields that

$$
\begin{aligned}
& \left|\int_{\mathbb{R}} \partial_{x_{1}}^{\gamma_{1}} \partial_{x_{2}}^{\gamma_{2}} K_{\left(\alpha_{1}, \alpha_{2}\right)}^{\sharp}\left(x_{1}, x_{2}, x_{3}\right) \varphi\left(\delta x_{3}\right) d x_{3}\right| \\
& \quad \leq C\left\{\sum_{k_{1}=-\infty}^{\infty} \sum_{k_{2}=-\infty}^{\infty}\left|1-2^{-k_{1} \alpha_{1}-k_{2} \alpha_{2}}\right| 2^{-\left|k_{1}\right|-\left|k_{2}\right|}\right\} \frac{1}{\left\|\left(x_{1}, x_{2}\right)\right\|^{3+\gamma_{1}+2 \gamma_{2}}},
\end{aligned}
$$

where $C$ is a positive constant independent of $\left(x_{1}, x_{2}\right) \in \mathbb{R}^{2}$ and $\alpha_{1}, \alpha_{2} \in \mathbb{Z}_{+}$. Similarly, we can show that for all normalized bump function $\varphi$ on $\mathbb{R}^{2}, \delta>0$, 
and all $x_{3} \in \mathbb{R}$,

$$
\begin{aligned}
& \left|\int_{\mathbb{R}^{2}} \partial_{x_{3}}^{\gamma_{3}} K_{\left(\alpha_{1}, \alpha_{2}\right)}^{\sharp}\left(x_{1}, x_{2}, x_{3}\right) \varphi\left(\delta x_{1}, \delta^{2} x_{2}\right) d x_{1} d x_{2}\right| \\
& \leq C\left\{\sum_{k_{1}=-\infty}^{\infty} \sum_{k_{2}=-\infty}^{\infty}\left|1-2^{-k_{1} \alpha_{1}-k_{2} \alpha_{2}}\right| 2^{-\left|k_{1}\right|-\left|k_{2}\right|}\right\} \frac{1}{\left|x_{3}\right|^{1+\gamma_{3}}},
\end{aligned}
$$

where $C$ is a positive constant independent of $x_{3} \in \mathbb{R}$ and $\alpha_{1}, \alpha_{2} \in \mathbb{Z}_{+}$. The estimates (34) and (35), and the special structure of $K_{\left(\alpha_{1}, \alpha_{2}\right)}^{\sharp}$ imply that for all normalized bump functions $\varphi_{1}$ and $\varphi_{2}$, respectively, on $\mathbb{R}^{2}$ and $\mathbb{R}$, and all $\delta_{1}, \delta_{2}>0$,

$$
\begin{aligned}
& \left|\int_{\mathbb{R}^{2} \times \mathbb{R}} K_{\left(\alpha_{1}, \alpha_{2}\right)}^{\sharp}\left(x_{1}, x_{2}, x_{3}\right) \varphi_{1}\left(\delta_{1} x_{1}, \delta_{1}^{2} x_{2}\right) \varphi_{2}\left(\delta_{2} x_{2}\right) d x_{1} d x_{2} d x_{3}\right| \\
& \leq C\left\{\sum_{k_{1}=-\infty}^{\infty} \sum_{k_{2}=-\infty}^{\infty}\left|1-2^{-k_{1} \alpha_{1}-k_{2} \alpha_{2}}\right| 2^{-\left|k_{1}\right|-\left|k_{2}\right|}\right\}
\end{aligned}
$$

with the positive constant $C$ independent of $\alpha_{1}, \alpha_{2} \in \mathbb{Z}_{+}$. Thus, the estimates (34), (35), (36), and (37) and Remark 1.3 imply that the kernel $K_{\left(\alpha_{1}, \alpha_{2}\right)}^{\sharp}$ is a product kernel on $\mathbb{R}^{2} \times \mathbb{R}$ with a constant no more than

$$
C_{0}\left\{\sum_{k_{1}=-\infty}^{\infty} \sum_{k_{2}=-\infty}^{\infty}\left|1-2^{-k_{1} \alpha_{1}-k_{2} \alpha_{2}}\right| 2^{-\left|k_{1}\right|-\left|k_{2}\right|}\right\}
$$

where $C_{0}$ is a positive constant independent of $\alpha_{1}, \alpha_{2} \in \mathbb{Z}_{+}$. Now, Theorem 3.1 and its proof imply that $I-I_{\left(-\alpha_{1},-\alpha_{2}\right)} I_{\left(\alpha_{1}, \alpha_{2}\right)}$ is bounded on $\dot{B}_{p q}^{s}\left(\mathbb{R}^{2}\right)$ and $\dot{F}_{p q}^{s}\left(\mathbb{R}^{2}\right)$ with operator norms no more than the quantity in (38). It is easy to see that we can choose $\alpha_{1}^{0}\left(s_{1}, s_{2}\right)>0$ and $\alpha_{2}^{0}\left(s_{1}, s_{2}\right)>0$ so small that if $\left|\alpha_{1}\right|<\alpha_{1}^{0}\left(s_{1}, s_{2}\right)$ and $\left|\alpha_{2}\right|<\alpha_{2}^{0}\left(s_{1}, s_{2}\right)$, then

$$
C_{0}\left\{\sum_{k_{1}=-\infty}^{\infty} \sum_{k_{2}=-\infty}^{\infty}\left|1-2^{-k_{1} \alpha_{1}-k_{2} \alpha_{2}}\right| 2^{-\left|k_{1}\right|-\left|k_{2}\right|}\right\}<1,
$$

where $C_{0}$ is the same positive constant as in (38). Thus, under this restriction, we know that $\left(I_{\left(-\alpha_{1},-\alpha_{2}\right)} I_{\left(\alpha_{1}, \alpha_{2}\right)}\right)^{-1}$ exists and is bounded, respectively, on $\dot{B}_{p q}^{s}\left(\mathbb{R}^{2}\right)$ with $p, q \in[1, \infty]$ and $\dot{F}_{p q}^{s}\left(\mathbb{R}^{2}\right)$ with $p \in(1, \infty)$ and $q \in(1, \infty]$. Namely, there exists a positive constant $C$ such that

$$
\left\|\left(I_{\left(-\alpha_{1},-\alpha_{2}\right)} I_{\left(\alpha_{1}, \alpha_{2}\right)}\right)^{-1}(f)\right\|_{\dot{B}_{p q}^{s}\left(\mathbb{R}^{2}\right)} \leq C\|f\|_{\dot{B}_{p q}^{s}\left(\mathbb{R}^{2}\right)}
$$

for all $f \in \dot{B}_{p q}^{s}\left(\mathbb{R}^{2}\right)$ with $p, q \in[1, \infty]$, and for all $f \in \dot{F}_{p q}^{s}\left(\mathbb{R}^{2}\right)$ with $p \in(1, \infty)$ and $q \in(1, \infty]$,

$$
\left\|\left(I_{\left(-\alpha_{1},-\alpha_{2}\right)} I_{\left(\alpha_{1}, \alpha_{2}\right)}\right)^{-1}(f)\right\|_{\dot{F}_{p q}^{s}\left(\mathbb{R}^{2}\right)} \leq C\|f\|_{\dot{F}_{p q}^{s}\left(\mathbb{R}^{2}\right)} .
$$


Combining these with Proposition 4.3 yields that, if $\left|\alpha_{1}\right|<\alpha_{1}^{0}\left(s_{1}, s_{2}\right)$ and $\left|\alpha_{2}\right|<$ $\alpha_{2}^{0}\left(s_{1}, s_{2}\right)$, then there exists a positive constant $C$ such that

$$
\begin{aligned}
\|f\|_{\dot{B}_{p q}^{s}\left(\mathbb{R}^{2}\right)} & =\left\|\left(I_{\left(-\alpha_{1},-\alpha_{2}\right)} I_{\left(\alpha_{1}, \alpha_{2}\right)}\right)^{-1}\left(I_{\left(-\alpha_{1},-\alpha_{2}\right)} I_{\left(\alpha_{1}, \alpha_{2}\right)}\right)(f)\right\|_{\dot{B}_{p q}^{s}\left(\mathbb{R}^{2}\right)} \\
& \leq C\left\|\left(I_{\left(-\alpha_{1},-\alpha_{2}\right)} I_{\left(\alpha_{1}, \alpha_{2}\right)}\right)(f)\right\|_{\dot{B}_{p q}^{s}\left(\mathbb{R}^{2}\right)} \leq C\left\|I_{\left(\alpha_{1}, \alpha_{2}\right)}(f)\right\|_{\dot{B}_{p q}^{s+\alpha}\left(\mathbb{R}^{2}\right)},
\end{aligned}
$$

for all $f \in \dot{B}_{p q}^{s}\left(\mathbb{R}^{2}\right)$ with $p, q \in[1, \infty]$, and for all $f \in \dot{F}_{p q}^{s}\left(\mathbb{R}^{2}\right)$ with $p \in(1, \infty)$ and $q \in(1, \infty]$,

$$
\begin{aligned}
\|f\|_{\dot{F}_{p q}^{s}\left(\mathbb{R}^{2}\right)} & =\left\|\left(I_{\left(-\alpha_{1},-\alpha_{2}\right)} I_{\left(\alpha_{1}, \alpha_{2}\right)}\right)^{-1}\left(I_{\left(-\alpha_{1},-\alpha_{2}\right)} I_{\left(\alpha_{1}, \alpha_{2}\right)}\right)(f)\right\|_{\dot{F}_{p q}^{s}\left(\mathbb{R}^{2}\right)} \\
& \leq C\left\|\left(I_{\left(-\alpha_{1},-\alpha_{2}\right)} I_{\left(\alpha_{1}, \alpha_{2}\right)}\right)(f)\right\|_{\dot{F}_{p q}^{s}\left(\mathbb{R}^{2}\right)} \leq C\left\|I_{\left(\alpha_{1}, \alpha_{2}\right)}(f)\right\|_{\dot{F}_{p q}^{s+\alpha}\left(\mathbb{R}^{2}\right)},
\end{aligned}
$$

which completes the proof of Proposition 4.5.

Combining Proposition 4.3 with Proposition 4.5 yields the following lifting properties of $\dot{B}_{p q}^{s}\left(\mathbb{R}^{2}\right)$ and $\dot{F}_{p q}^{s}\left(\mathbb{R}^{2}\right)$.

Theorem 4.6. Let $\left|s_{i}\right|<1,\left|s_{i}+\alpha_{i}\right|<1$ for $i=1,2, s=\left(s_{1}, s_{2}\right)$ and $s+\alpha=$ $\left(s_{1}+\alpha_{1}, s_{2}+\alpha_{2}\right)$. Then there exist a positive constant $C$ and $\alpha_{i}^{0}\left(s_{1}, s_{2}\right) \in(0,1)$ such that, if $\left|\alpha_{i}\right|<\alpha_{i}^{0}\left(s_{1}, s_{2}\right)$ with $i=1,2$,

$$
C^{-1}\|f\|_{\dot{B}_{p q}^{s}\left(\mathbb{R}^{2}\right)} \leq\left\|I_{\left(\alpha_{1}, \alpha_{2}\right)}(f)\right\|_{\dot{B}_{p q}^{s+\alpha}\left(\mathbb{R}^{2}\right)} \leq C\|f\|_{\dot{B}_{p q}^{s}\left(\mathbb{R}^{2}\right)}
$$

for all $f \in \dot{B}_{p q}^{s}\left(\mathbb{R}^{2}\right)$ with $p, q \in[1, \infty]$, and for all $f \in \dot{F}_{p q}^{s}\left(\mathbb{R}^{2}\right)$ with $p \in(1, \infty)$ and $q \in(1, \infty]$,

$$
C^{-1}\|f\|_{\dot{F}_{p q}^{s}\left(\mathbb{R}^{2}\right)} \leq\left\|I_{\left(\alpha_{1}, \alpha_{2}\right)}(f)\right\|_{\dot{F}_{p q}^{s+\alpha}\left(\mathbb{R}^{2}\right)} \leq C\|f\|_{\dot{F}_{p q}^{s}\left(\mathbb{R}^{2}\right)} .
$$

\section{Embedding theorems and fractional integrals}

In this section we first present some embedding theorems for both Besov spaces $\dot{B}_{p q}^{s}\left(\mathbb{R}^{2}\right)$ and Triebel-Lizorkin spaces $\dot{F}_{p q}^{s}\left(\mathbb{R}^{2}\right)$. We remark that our embedding theorems for Triebel-Lizorkin spaces are not the same as those for Besov spaces, which reflects the difference between these two kinds of spaces. As an application, we then obtain another boundedness of fractional integrals $I_{\left(\alpha_{1}, \alpha_{2}\right)}$ on $\dot{B}_{p q}^{s}\left(\mathbb{R}^{2}\right)$ and $\dot{F}_{p q}^{s}\left(\mathbb{R}^{2}\right)$ again.

Theorem 5.1. Let $q \in[1, \infty], p_{1}, p_{2} \in[1, \infty],\left|s_{i}\right|<1,\left|\bar{s}_{i}\right|<1$, and $\bar{s}_{i}<s_{i}$ with $i=1,2$. Then

(i) If $s_{1}-3 / p_{1}=\bar{s}_{1}-3 / p_{2}$, then $\dot{B}_{p_{1}, q}^{\left(s_{1}, s_{2}\right)}\left(\mathbb{R}^{2}\right) \subset \dot{B}_{p_{2}, q}^{\left(\bar{s}_{1}, s_{2}\right)}\left(\mathbb{R}^{2}\right)$, namely, there exists a positive constant $C$ such that, for all $f \in \dot{B}_{p_{1}, q}^{\left(s_{1}, s_{2}\right)}\left(\mathbb{R}^{2}\right)$,

$$
\|f\|_{\dot{B}_{p_{2}, q}^{\left(\bar{s}_{1}, s_{2}\right)}\left(\mathbb{R}^{2}\right)} \leq C\|f\|_{\dot{B}_{p_{1}, q}^{\left(s_{1}, s_{2}\right)}\left(\mathbb{R}^{2}\right)} .
$$


(ii) If $\bar{s}_{1}-s_{1}=1 / p_{2}-1 / p_{1}=\bar{s}_{2}-s_{2}$, then $\dot{B}_{p_{1}, q}^{\left(s_{1}, s_{2}\right)}\left(\mathbb{R}^{2}\right) \subset \dot{B}_{p_{2}, q}^{\left(\bar{s}_{1}, \bar{s}_{2}\right)}\left(\mathbb{R}^{2}\right)$, namely, there exists a positive constant $C$ such that, for all $f \in \dot{B}_{p_{1}, q}^{\left(s_{1}, s_{2}\right)}\left(\mathbb{R}^{2}\right)$,

$$
\|f\|_{\dot{B}_{p_{2}, q}^{\left(\bar{s}_{1}, \bar{s}_{2}\right)}\left(\mathbb{R}^{2}\right)} \leq C\|f\|_{\dot{B}_{p_{1}, q}^{\left(s_{1}, s_{2}\right)}\left(\mathbb{R}^{2}\right)} .
$$

Proof. To simplify our proof, based on Theorem 2.8 and Theorem 2.6, we may suppose that $\psi^{(i)}$ for $i=1,2$ in Lemma 2.4 have compact supports, namely,

$$
\operatorname{supp} \psi^{(1)} \subset\left\{\left(x_{1}, x_{2}\right) \in \mathbb{R}^{2}:\left\|\left(x_{1}, x_{2}\right)\right\| \leq 1\right\}
$$

and $\operatorname{supp} \psi^{(2)} \subset\left\{x_{3} \in \mathbb{R}:\left|x_{3}\right| \leq 1\right\}$. Let other notation be the same as in Lemma 2.4. We then easily show that for all $j_{1}, k_{1} \in \mathbb{Z},\left(x_{1}, x_{2}\right) \in \mathbb{R}^{2}$, and $x_{3} \in \mathbb{R}$,

$$
\begin{gathered}
\operatorname{supp} \psi_{j_{1}}^{(1)} * \psi_{k_{1}}^{(1)} \subset\left\{\left(x_{1}, x_{2}\right) \in \mathbb{R}^{2}:\left\|\left(x_{1}, x_{2}\right)\right\| \leq 2^{1-\left(j_{1} \wedge k_{1}\right)}\right\}, \\
\left|\left(\psi_{j_{1}}^{(1)} * \psi_{k_{1}}^{(1)}\right)\left(x_{1}, x_{2}\right)\right| \leq C 2^{-\left|j_{1}-k_{1}\right|} 2^{3\left(j_{1} \wedge k_{1}\right)}
\end{gathered}
$$

and

$$
\begin{gathered}
\operatorname{supp} \psi_{j_{2}}^{(2)} * \psi_{k_{2}}^{(2)} \subset\left\{x_{3} \in \mathbb{R}:\left|x_{3}\right| \leq 2^{1-\left(j_{2} \wedge k_{2}\right)}\right\}, \\
\left|\left(\psi_{j_{2}}^{(2)} * \psi_{k_{2}}^{(2)}\right)\left(x_{3}\right)\right| \leq C 2^{-\left|j_{2}-k_{2}\right|} 2^{j_{2} \wedge k_{2}} .
\end{gathered}
$$

The estimates (39) and (40), Lemma 2.4, and the Hölder inequality yield that for all $j_{1}, j_{2} \in \mathbb{Z}$ and $\left(x_{1}, x_{2}\right) \in \mathbb{R}^{2}$

$$
\begin{aligned}
\left|\psi_{j_{1} j_{2}} * f\left(x_{1}, x_{2}\right)\right|=\mid & \sum_{k_{1}, k_{2} \in \mathbb{Z}} \int_{\mathbb{R}^{2}}\left(\psi_{j_{1}}^{(1)} * \psi_{k_{1}}^{(1)}\right) *_{2}\left(\psi_{j_{2}}^{(2)} * \psi_{k_{2}}^{(2)}\right)\left(x_{1}-y_{1}, x_{2}-y_{2}\right) \\
& \times \psi_{k_{1} k_{2}} * f\left(y_{1}, y_{2}\right) d y_{1} d y_{2} \mid \\
\leq & C \sum_{k_{1}, k_{2} \in \mathbb{Z}} 2^{-\left|j_{1}-k_{1}\right| / p_{1}^{\prime}-\left|j_{2}-k_{2}\right| / p_{1}^{\prime}} \\
& \times\left\{\int_{\mathbb{R}^{2}}\left|\left(\psi_{j_{1}}^{(1)} * \psi_{k_{1}}^{(1)}\right) *_{2}\left(\psi_{j_{2}}^{(2)} * \psi_{k_{2}}^{(2)}\right)\left(x_{1}-y_{1}, x_{2}-y_{2}\right)\right|\right. \\
& \left.\times\left|\left(\psi_{k_{1} k_{2}} * f\right)\left(y_{1}, y_{2}\right)\right|^{p_{1}} d y_{1} d y_{2}\right\}^{1 / p_{1}} .
\end{aligned}
$$

Noticing that $p_{2}>p_{1}$, by the above estimate and the Minkowski inequality, we obtain

$$
\begin{aligned}
& \left\|\psi_{j_{1} j_{2}} * f\right\|_{L^{p_{2}\left(\mathbb{R}^{2}\right)}} \\
& \leq C \sum_{k_{1}, k_{2} \in \mathbb{Z}} 2^{-\left|j_{1}-k_{1}\right| / p_{1}^{\prime}-\left|j_{2}-k_{2}\right| / p_{1}^{\prime}}\left\|\psi_{k_{1} k_{2}} * f\right\|_{L^{p_{1}}\left(\mathbb{R}^{2}\right)} \\
& \quad \times\left\{\int_{\mathbb{R}^{2}}\left|\left(\psi_{j_{1}}^{(1)} * \psi_{k_{1}}^{(1)}\right) *_{2}\left(\psi_{j_{2}}^{(2)} * \psi_{k_{2}}^{(2)}\right)\left(x_{1}, x_{2}\right)\right|^{p_{2} / p_{1}} d x_{1} d x_{2}\right\}^{p_{1} / p_{2}} .
\end{aligned}
$$


We now have two ways to estimate the last quantity in (41), which result in the conclusions (i) and (ii) of Theorem 5.1, respectively.

Proof of (i). The Minkowski inequality and the estimates (39) and (40) imply that

$$
\begin{aligned}
\left\{\int_{\mathbb{R}^{2}}\left|\left(\psi_{j_{1}}^{(1)} * \psi_{k_{1}}^{(1)}\right) *_{2}\left(\psi_{j_{2}}^{(2)} * \psi_{k_{2}}^{(2)}\right)\left(x_{1}, x_{2}\right)\right|^{p_{2} / p_{1}} d x_{1} d x_{2}\right\}^{p_{1} / p_{2}} & \\
& \leq C 2^{-\left|j_{1}-k_{1}\right|} 2^{3\left(j_{1} \wedge k_{1}\right)\left(1-p_{1} / p_{2}\right)} 2^{-\left|j_{2}-k_{2}\right|} .
\end{aligned}
$$

Inserting this in (41) leads us to

$$
\begin{aligned}
& \left\|\psi_{j_{1} j_{2}} * f\right\|_{L^{p_{2}\left(\mathbb{R}^{2}\right)}} \\
& \leq C \sum_{k_{1}, k_{2} \in \mathbb{Z}} 2^{-\left|j_{1}-k_{1}\right|-\left|j_{2}-k_{2}\right|} 2^{3\left(j_{1} \wedge k_{1}\right)\left(1 / p_{1}-1 / p_{2}\right)}\left\|\psi_{k_{1} k_{2}} * f\right\|_{L^{p_{1}}\left(\mathbb{R}^{2}\right)},
\end{aligned}
$$

which together with Lemma 2.4 and the assumption that $s_{1}-3 / p_{1}=\bar{s}_{1}-3 / p_{2}$ yields that

$$
\begin{aligned}
\|f\|_{\dot{B}_{p_{2}, q}^{\left(\bar{s}_{1}, s_{2}\right)}\left(\mathbb{R}^{2}\right)} & \\
\leq C & \left\{\sum_{j_{1}, j_{2} \in \mathbb{Z}} 2^{j_{1} \bar{s}_{1} q} 2^{j_{2} s_{2} q}\right. \\
& \left.\times\left[\sum_{k_{1}, k_{2} \in \mathbb{Z}} 2^{-\left|j_{1}-k_{1}\right|-\left|j_{2}-k_{2}\right|} 2^{3\left(j_{1} \wedge k_{1}\right)\left(1 / p_{1}-1 / p_{2}\right)}\left\|\psi_{k_{1} k_{2}} * f\right\|_{L^{p_{1}\left(\mathbb{R}^{2}\right)}}\right]^{q}\right\}^{1 / q} \\
\leq & C\left\{\sum_{k_{1}, k_{2} \in \mathbb{Z}} 2^{k_{1} s_{1} q} 2^{k_{2} s_{2} q}\left\|\psi_{k_{1} k_{2}} * f\right\|_{L^{p_{1}\left(\mathbb{R}^{2}\right)}}^{q}\right\}^{1 / q} \leq C\|f\|_{\dot{B}_{p_{1}, q}^{\left(s_{1}, s_{2}\right)}\left(\mathbb{R}^{2}\right)},
\end{aligned}
$$

where in the second-to-last inequality, we used the assumptions that $\bar{s}_{1}<1, s_{1}>-1$, and $\left|s_{2}\right|<1$. This proves (i) of Theorem 5.1.

Proof of (ii). Again, the Minkowski inequality and the estimates (39) and (40) yield that

$$
\begin{aligned}
\left\{\int_{\mathbb{R}^{2}} \mid\left(\psi_{j_{1}}^{(1)} * \psi_{k_{1}}^{(1)}\right)\right. & \left.\left.*_{2}\left(\psi_{j_{2}}^{(2)} * \psi_{k_{2}}^{(2)}\right)\left(x_{1}, x_{2}\right)\right|^{p_{2} / p_{1}} d x_{1} d x_{2}\right\}^{p_{1} / p_{2}} \\
\leq & \left\{\int _ { \mathbb { R } } \left[\int_{\mathbb{R}}\left|\left(\psi_{j_{1}}^{(1)} * \psi_{k_{1}}^{(1)}\right)\left(x_{1}, x_{3}\right)\right|\right.\right. \\
& \left.\left.\times\left\{\int_{\mathbb{R}}\left|\left(\psi_{j_{2}}^{(2)} * \psi_{k_{2}}^{(2)}\right)(z)\right|^{p_{2} / p_{1}} d z\right\}^{p_{1} / p_{2}} d x_{3}\right]^{p_{2} / p_{1}} d x_{1}\right\}^{p_{1} / p_{2}} \\
\leq & C 2^{\left(j_{1} \wedge k_{1}\right)\left(1-p_{1} / p_{2}\right)-\left|j_{1}-k_{1}\right|} 2^{\left(j_{2} \wedge k_{2}\right)\left(1-p_{1} / p_{2}\right)-\left|j_{2}-k_{2}\right|} .
\end{aligned}
$$


This, Lemma 2.4, and the assumptions that $\bar{s}_{1}-s_{1}=1 / p_{2}-1 / p_{1}=\bar{s}_{2}-s_{2}$ lead us to

$$
\begin{aligned}
& \|f\|_{\dot{B}_{p_{2}, \bar{s}_{1}}^{\left(\bar{s}_{1}, \bar{s}_{2}\right)}\left(\mathbb{R}^{2}\right)} \leq C\left\{\sum _ { j _ { 1 } , j _ { 2 } \in \mathbb { Z } } 2 ^ { j _ { 1 } \overline { s } _ { 1 } q } 2 ^ { j _ { 2 } \overline { s } _ { 2 } q } \left[\sum_{k_{1}, k_{2} \in \mathbb{Z}} 2^{\left(j_{1} \wedge k_{1}\right)\left(1 / p_{1}-1 / p_{2}\right)-\left|j_{1}-k_{1}\right|}\right.\right. \\
& \left.\left.\times 2^{\left(j_{2} \wedge k_{2}\right)\left(1 / p_{1}-1 / p_{2}\right)-\left|j_{2}-k_{2}\right|}\left\|\psi_{k_{1} k_{2}} * f\right\|_{L^{p_{1}}\left(\mathbb{R}^{2}\right)}\right]^{q}\right\}^{1 / q} \\
& \leq C\left\{\sum_{k_{1}, k_{2} \in \mathbb{Z}} 2^{k_{1} s_{1} q} 2^{k_{2} s_{2} q}\left\|\psi_{k_{1} k_{2}} * f\right\|_{L^{p_{1}}\left(\mathbb{R}^{2}\right)}^{q}\right\}^{1 / q} \\
& \leq C\|f\|_{\dot{B}_{p_{1}, q}^{\left(s_{1}, s_{2}\right)}\left(\mathbb{R}^{2}\right)},
\end{aligned}
$$

where in the second-to-last inequality, we used the assumptions that $\bar{s}_{i}<1$ and $s_{i}>-1$ for $i=1,2$, which proves (ii) of Theorem 5.1. This finishes the proof of Theorem 5.1.

We now establish an embedding theorem for Triebel-Lizorkin spaces $\dot{F}_{p q}^{s}\left(\mathbb{R}^{2}\right)$.

Theorem 5.2. Let $q_{i} \in(1, \infty], p_{i} \in(1, \infty),\left|s_{i}\right|<1$, and $\left|\bar{s}_{i}\right|<1$ with $i=1,2$. Assume that $s_{2}<\bar{s}_{2}, \bar{s}_{1}+2 \bar{s}_{2}<s_{1}+2 s_{2}$, and $s_{1}+2 s_{2}-3 / p_{1}=\bar{s}_{1}+2 \bar{s}_{2}-3 / p_{2}$. Then $\dot{F}_{p_{1}, q_{1}}^{\left(s_{1}, s_{2}\right)}\left(\mathbb{R}^{2}\right) \subset \dot{F}_{p_{2}, q_{2}}^{\left(\bar{s}_{1}, \bar{s}_{2}\right)}\left(\mathbb{R}^{2}\right)$, namely, there exists a positive constant $C$ such that, for all $f \in \dot{F}_{p_{1}, q_{1}}^{\left(s_{1}, s_{2}\right)}\left(\mathbb{R}^{2}\right)$,

$$
\|f\|_{\dot{F}_{p_{2}, q_{2}}^{\left(\bar{s}_{1}, \bar{s}_{2}\right)\left(\mathbb{R}^{2}\right)}} \leq C\|f\|_{\dot{F}_{p_{1}, q_{1}}^{\left(s_{1}, s_{2}\right)}\left(\mathbb{R}^{2}\right)} .
$$

Proof. By Proposition 2.9 (ii), we may assume that $q_{1}=\infty$ and $q_{2}=1$. Thus, to prove the theorem, we only need to show $\dot{F}_{p_{1}, \infty}^{\left(s_{1}, s_{2}\right)}\left(\mathbb{R}^{2}\right) \subset \dot{F}_{p_{2}, 1}^{\left(\bar{s}_{1}, \bar{s}_{2}\right)}\left(\mathbb{R}^{2}\right)$. Let $f \in \dot{F}_{p_{1}, \infty}^{\left(s_{1}, s_{2}\right)}\left(\mathbb{R}^{2}\right)$ and $\|f\|_{\dot{F}_{p_{1}, \infty}^{\left(s_{1}, s_{2}\right)}\left(\mathbb{R}^{2}\right)}=1$ by homogeneity. We also let $\psi^{(i)}$ for $i=1,2$ be the same as in the proof of Corollary 2.22. Using the same notation as in Lemma 2.4, by Lemma 2.4 and this special choice of $\psi^{(i)}$ for $i=1,2$, we then have that

$$
f\left(x_{1}, x_{2}\right)=\sum_{j_{1}=-\infty}^{\infty} \sum_{j_{2}=-\infty}^{2 j_{1}+3} \psi_{j_{1} j_{2}} * \psi_{j_{1} j_{2}} * f\left(x_{1}, x_{2}\right)
$$

holds with the same meaning as in (4). By (42) and the Hölder inequality, we have that, for $k_{1}, k_{2} \in \mathbb{Z}$ and $\left(x_{1}, x_{2}\right) \in \mathbb{R}^{2}$,

$$
\begin{array}{r}
\left|\psi_{k_{1} k_{2}} * f\left(x_{1}, x_{2}\right)\right| \leq \sum_{j_{1}=-\infty}^{\infty} \sum_{j_{2}=-\infty}^{2 j_{1}+3}\left\{\int_{\mathbb{R}^{2}}\left|\left(\psi_{k_{1} k_{2}} * \psi_{j_{1} j_{2}}\right)\left(y_{1}, y_{2}\right)\right|^{p_{1}^{\prime}} d y_{1} d y_{2}\right\}^{1 / p_{1}^{\prime}} \\
\times\left\|\psi_{j_{1} j_{2}} * f\right\|_{L^{p_{1}}\left(\mathbb{R}^{2}\right)}
\end{array}
$$


The estimate (32) and the Minkowski inequality imply that

$$
\begin{aligned}
& \left\{\int_{\mathbb{R}^{2}}\left|\left(\psi_{k_{1} k_{2}} * \psi_{j_{1} j_{2}}\right)\left(y_{1}, y_{2}\right)\right|^{p_{1}^{\prime}} d y_{1} d y_{2}\right\}^{1 / p_{1}^{\prime}} \\
& \leq C 2^{-\left|j_{2}-k_{2}\right|} 2^{-\left|j_{1}-k_{1}\right|}\left\{\int_{\mathbb{R}^{2}} \frac{2^{-\left(j_{1} \wedge k_{1}\right) p_{1}^{\prime}}}{\left(2^{-\left(j_{1} \wedge k_{1}\right)}+\left\|\left(y_{1}, y_{2}\right)\right\|\right)^{4 p_{1}^{\prime}}} d y_{1} d y_{2}\right\}^{1 / p_{1}^{\prime}} \\
& \leq C 2^{-\left|j_{2}-k_{2}\right|} 2^{3\left(j_{1} \wedge k_{1}\right) / p_{1}-\left|j_{1}-k_{1}\right|} \text {. }
\end{aligned}
$$

Combining this estimate with (43) and using the assumption that $\|f\|_{\dot{F}_{p_{1}, \infty}^{\left(s_{1}, s_{2}\right)}\left(\mathbb{R}^{2}\right)}=1$ yield that

$$
\left|\psi_{k_{1} k_{2}} * f\left(x_{1}, x_{2}\right)\right| \leq C \sum_{j_{1}=-\infty}^{\infty} \sum_{j_{2}=-\infty}^{2 j_{1}+3} 2^{-\left|j_{2}-k_{2}\right|-j_{2} s_{2}} 2^{3\left(j_{1} \wedge k_{1}\right) / p_{1}-\left|j_{1}-k_{1}\right|-j_{1} s_{1}}
$$

From this and the assumptions that $\bar{s}_{2}>s_{2}, s_{1}+2 s_{2}-3 / p_{1}=\bar{s}_{1}+2 \bar{s}_{2}-3 / p_{2}$, and $\left|s_{i}\right|<1$ for $i=1,2$, it follows that, for any fixed $N \in \mathbb{Z}$ and all $\left(x_{1}, x_{2}\right) \in \mathbb{R}^{2}$,

$$
\begin{aligned}
\sum_{k_{1}=-\infty}^{N} \sum_{k_{2}=-\infty}^{2 k_{1}+3} 2^{k_{1} \bar{s}_{1}} 2^{k_{2} \bar{s}_{2}}\left|\psi_{k_{1} k_{2}} * f\left(x_{1}, x_{2}\right)\right| \\
\leq C \sum_{k_{1}=-\infty}^{N} \sum_{k_{2}=-\infty}^{2 k_{1}+3} 2^{k_{1} \bar{s}_{1}} 2^{k_{2}\left(\bar{s}_{2}-s_{2}\right)} \\
\quad \times \sum_{j_{1}=-\infty}^{\infty} \sum_{j_{2}=-\infty}^{2 j_{1}+3} 2^{\left(k_{2}-j_{2}\right) s_{2}-\left|j_{2}-k_{2}\right|} 2^{3\left(j_{1} \wedge k_{1}\right) / p_{1}-\left|j_{1}-k_{1}\right|-j_{1} s_{1}} \\
\leq C_{0} 2^{3 N / p_{2}}
\end{aligned}
$$

where $C_{0}$ is a positive constant independent of $N \in \mathbb{Z}$. On the other hand, for all $N \in \mathbb{Z}$ and $\left(x_{1}, x_{2}\right) \in \mathbb{R}^{2}$, by the assumptions that $s_{2}<\bar{s}_{2}$ and $\bar{s}_{1}+2 \bar{s}_{2}<s_{1}+2 s_{2}$, we have

$$
\begin{aligned}
& \sum_{k_{1}=N+1}^{\infty} \sum_{k_{2}=-\infty}^{2 k_{1}+3} 2^{k_{1} \bar{s}_{1}} 2^{k_{2} \bar{s}_{2}}\left|\psi_{k_{1} k_{2}} * f\left(x_{1}, x_{2}\right)\right| \\
& \leq \sum_{k_{1}=N+1}^{\infty} \sum_{k_{2}=-\infty}^{2 k_{1}+3} 2^{k_{1}\left(\bar{s}_{1}-s_{1}\right)} 2^{k_{2}\left(\bar{s}_{2}-s_{2}\right)} \sup _{\substack{j_{1} \in \mathbb{Z} \\
-\infty<j_{2} \leq 2 j_{1}+3}} 2^{j_{1} s_{1}} 2^{j_{2} s_{2}}\left|\psi_{j_{1} j_{2}} * f\left(x_{1}, x_{2}\right)\right| \\
& \leq C_{1} 2^{N\left(\bar{s}_{1}+2 \bar{s}_{2}-s_{1}-2 s_{2}\right)} \sup _{\substack{j_{1} \in \mathbb{Z} \\
-\infty<j_{2} \leq 2 j_{1}+3}} 2^{j_{1} s_{1}} 2^{j_{2} s_{2}}\left|\psi_{j_{1} j_{2}} * f\left(x_{1}, x_{2}\right)\right|
\end{aligned}
$$


where the positive constant $C_{1}$ is independent of $N \in \mathbb{Z}$. The estimates (44) and (45) then imply that

$$
\begin{aligned}
& \|f\|_{\dot{F}_{p_{2}, 1}^{\left(\bar{s}_{1}, \bar{s}_{2}\right)}\left(\mathbb{R}^{2}\right)}^{p_{2}}=p_{2} \sum_{N=-\infty}^{\infty} \int_{C_{0} 2^{3 N / p_{2}}}^{C_{0} 2^{3(N+1) / p_{2}}} t^{p_{2}-1} \\
& \times \mid\left\{\left(x_{1}, x_{2}\right) \in \mathbb{R}^{2}: \sum_{k_{1}=-\infty}^{N} \sum_{k_{2}=-\infty}^{2 k_{1}+3} 2^{k_{1} \bar{s}_{1}} 2^{k_{2} \bar{s}_{2}}\left|\psi_{k_{1} k_{2}} * f\left(x_{1}, x_{2}\right)\right|\right. \\
& \left.+\sum_{k_{1}=N+1}^{\infty} \sum_{k_{2}=-\infty}^{2 k_{1}+3} \cdots>t\right\} \mid d t \\
& \leq C p_{2} \int_{0}^{\infty} t^{p_{1}-1} \mid\left\{\left(x_{1}, x_{2}\right) \in \mathbb{R}^{2}: \sup _{\substack{j_{1} \in \mathbb{Z} \\
-\infty<j_{2} \leq 2 j_{1}+3}} 2^{j_{1} s_{1}} 2^{j_{2} s_{2}}\right. \\
& \left.\times\left|\psi_{j_{1} j_{2}} * f\left(x_{1}, x_{2}\right)\right|>t\right\} \mid d t \\
& =C\|f\|_{\dot{F}_{p_{1}, \infty}^{\left(s_{1}, s_{2}\right)}\left(\mathbb{R}^{2}\right)}^{p_{1}}=C,
\end{aligned}
$$

where the positive constant $C$ is independent of $f$. This finishes the proof of Theorem 5.2.

As a corollary of Proposition 4.3 and Theorems 5.1 and 5.2, we have the following boundedness of $I_{\left(\alpha_{1}, \alpha_{2}\right)}$ on $\dot{B}_{p q}^{s}\left(\mathbb{R}^{2}\right)$ and $\dot{F}_{p q}^{s}\left(\mathbb{R}^{2}\right)$.

Corollary 5.3. Let $\left|s_{i}\right|<1,\left|\alpha_{i}\right|<1$, and $\left|s_{i}+\alpha_{i}\right|<1$ for $i=1,2$. Then,

(i) If $q \in[1, \infty], \alpha_{1}>0, p_{1} \in(1, \infty)$, and $1 / p_{2}=1 / p_{1}-\alpha_{1} / 3$, then $I_{\left(\alpha_{1}, \alpha_{2}\right)}$ is bounded from $\dot{B}_{p_{1}, q}^{\left(s_{1}, s_{2}\right)}\left(\mathbb{R}^{2}\right)$ to $\dot{B}_{p_{2}, q}^{\left(s_{1}, s_{2}+\alpha_{2}\right)}\left(\mathbb{R}^{2}\right)$, namely, there exists a positive constant $C$ such that, for all $f \in \dot{B}_{p_{1}, q}^{\left(s_{1}, s_{2}\right)}\left(\mathbb{R}^{2}\right)$,

$$
\left\|I_{\left(\alpha_{1}, \alpha_{2}\right)}(f)\right\|_{\dot{B}_{p_{2}, q}^{\left(s_{1}, s_{2}+\alpha_{2}\right)}\left(\mathbb{R}^{2}\right)} \leq C\|f\|_{\dot{B}_{p_{1}, q}^{\left(s_{1}, s_{2}\right)}\left(\mathbb{R}^{2}\right)} .
$$

(ii) If $q \in[1, \infty], 0<\alpha_{1}=\alpha_{2}<1, p_{1} \in(1, \infty)$, and $1 / p_{2}=1 / p_{1}-\alpha_{1}$, then $I_{\left(\alpha_{1}, \alpha_{2}\right)}$ is bounded from $\dot{B}_{p_{1}, q}^{\left(s_{1}, s_{2}\right)}\left(\mathbb{R}^{2}\right)$ to $\dot{B}_{p_{2}, q}^{\left(s_{1}, s_{2}\right)}\left(\mathbb{R}^{2}\right)$, namely, there exists a positive constant $C$ such that, for all $f \in \dot{B}_{p_{1}, q}^{\left(s_{1}, s_{2}\right)}\left(\mathbb{R}^{2}\right)$,

$$
\left\|I_{\left(\alpha_{1}, \alpha_{2}\right)}(f)\right\|_{\dot{B}_{p_{2}, q}^{\left(s_{1}, s_{2}\right)}\left(\mathbb{R}^{2}\right)} \leq C\|f\|_{\dot{B}_{p_{1}, q}^{\left(s_{1}, s_{2}\right)}\left(\mathbb{R}^{2}\right)} .
$$

(iii) If $q_{1}, q_{2} \in(1, \infty], \alpha_{2}<0, \alpha_{1}>-2 \alpha_{2}, p_{1} \in(1, \infty)$, and $1 / p_{2}=1 / p_{1}-$ $\left(\alpha_{1}+2 \alpha_{2}\right) / 3$, then $I_{\left(\alpha_{1}, \alpha_{2}\right)}$ is bounded from $\dot{F}_{p_{1}, q_{1}}^{\left(s_{1}, s_{2}\right)}\left(\mathbb{R}^{2}\right)$ to $\dot{F}_{p_{2}, q_{2}}^{\left(s_{1}, s_{2}\right)}\left(\mathbb{R}^{2}\right)$, namely, there exists a positive constant $C$ such that, for all $f \in \dot{F}_{p_{1}, q_{1}}^{\left(s_{1}, s_{2}\right)}\left(\mathbb{R}^{2}\right)$,

$$
\left\|I_{\left(\alpha_{1}, \alpha_{2}\right)}(f)\right\|_{\dot{F}_{p_{2}, q_{2}}^{\left(s_{1}, s_{2}\right)\left(\mathbb{R}^{2}\right)}} \leq C\|f\|_{\dot{F}_{p_{1}, q_{1}}^{\left(s_{1}, s_{2}\right)}\left(\mathbb{R}^{2}\right)} .
$$


Finally, we consider the boundedness of semi-fractional integrals determined by the kernel $K_{\gamma}$ on $\dot{B}_{p q}^{s}\left(\mathbb{R}^{2}\right)$ and $\dot{F}_{p q}^{s}\left(\mathbb{R}^{2}\right)$.

Theorem 5.4. Let $K_{0}$ be a distribution kernel on $\mathbb{R}$ which coincides with a $C^{\infty}$ function away from $\{0\}$ and which satisfies:

(i) For any $\alpha \in \mathbb{Z}_{+}$, there exists a positive constant $C_{\alpha}$ such that, for all $z \in \mathbb{R} \backslash\{0\}$,

$$
\left|\partial_{z}^{\alpha} K_{0}(z)\right| \leq C_{\alpha}|z|^{-1-\alpha} .
$$

(ii) For any given normalized bump function $\varphi$ on $\mathbb{R}$ and any $\delta>0$, there exists a positive constant $C$ such that

$$
\left|\int_{\mathbb{R}} K_{0}(z) \varphi(\delta z) d z\right| \leq C
$$

For $\gamma \in(0,3)$, define the distribution kernel

$$
K_{\gamma}\left(x_{1}, x_{2}\right)=\left(\left\|\left(x_{1}, \cdot\right)\right\|^{\gamma-3} *_{2} K_{0}\right)\left(x_{2}\right),
$$

where $\left(x_{1}, x_{2}\right) \in \mathbb{R}^{2}$. Let $\left|s_{i}\right|<1$ for $i=1$, 2. If $p_{1} \in(1, \infty)$ and $1 / p_{2}=1 / p_{1}-\gamma / 3$, then there exists a positive constant $C$ such that, for all $f \in \dot{B}_{p_{1}, q}^{\left(s_{1}, s_{2}\right)}\left(\mathbb{R}^{2}\right)$ with $q \in[1, \infty]$,

$$
\left\|K_{\gamma} * f\right\|_{\dot{B}_{p_{2}, q}^{\left(s_{1}, s_{2}\right)}\left(\mathbb{R}^{2}\right)} \leq C\|f\|_{\dot{B}_{p_{1}, q}^{\left(s_{1}, s_{2}\right)}\left(\mathbb{R}^{2}\right)},
$$

and for all $f \in \dot{F}_{p_{1}, q}^{\left(s_{1}, s_{2}\right)}\left(\mathbb{R}^{2}\right)$ with $q \in(1, \infty]$,

$$
\left\|K_{\gamma} * f\right\|_{\dot{F}_{p_{2}, q}^{\left(s_{1}, s_{2}\right)}\left(\mathbb{R}^{2}\right)} \leq C\|f\|_{\dot{F}_{p_{1}, q}^{\left(s_{1}, s_{2}\right)}\left(\mathbb{R}^{2}\right)}
$$

Proof. Let $\psi^{(i)}$ for $i=1,2$ be the same as in the proof of Theorem 3.1. Repeating the proof of Theorem 3.1 implies that

$$
\begin{aligned}
&\left|\left(\psi_{u_{1} u_{2}} * K_{\gamma} * \psi_{t_{1} t_{2}}\right)\left(x_{1}, x_{2}\right)\right| \\
& \leq C\left(\frac{u_{1}}{t_{1}} \wedge \frac{t_{1}}{u_{1}}\right)\left(\frac{u_{2}}{t_{2}} \wedge \frac{t_{2}}{u_{2}}\right) \\
& \times \int_{\mathbb{R}} \frac{u_{1} \vee t_{1}}{\left(u_{1} \vee t_{1}+\left\|\left(x_{1}, x_{2}-x_{3}\right)\right\|\right)^{4-\gamma}} \frac{u_{2} \vee t_{2}}{\left(u_{2} \vee t_{2}+\left|x_{3}\right|\right)^{2}} d x_{3} .
\end{aligned}
$$

Inserting this estimate into (23) yields that

$$
\begin{aligned}
& \left|\left(\psi_{u_{1} u_{2}} * K_{\gamma} * f\right)\left(x_{1}, x_{2}\right)\right| \\
& \quad \leq C \int_{0}^{\infty} \int_{0}^{\infty}\left(\frac{u_{1}}{t_{1}} \wedge \frac{t_{1}}{u_{1}}\right)\left(\frac{u_{2}}{t_{2}} \wedge \frac{t_{2}}{u_{2}}\right) I_{\gamma} \circ M_{2}\left(\left|\left(\psi_{t_{1} t_{2}} * f\right)\left(x_{1}, \cdot\right)\right|\right)\left(x_{2}\right) \frac{d t_{1}}{t_{1}} \frac{d t_{2}}{t_{2}},
\end{aligned}
$$


where $I_{\gamma}$ is the standard fractional integral on $\mathbb{R}^{2}$ when $\mathbb{R}^{2}$ is regarded as a space of homogeneous type, and $M_{2}$ is the usual Hardy-Littlewood maximal function on the second variable. It is well-known that $I_{\gamma}$ is bounded from $L^{p_{1}}\left(\mathbb{R}^{2}\right)$ into $L^{p_{2}}\left(\mathbb{R}^{2}\right)$, where $p_{1} \in(1, \infty)$ and $1 / p_{2}=1 / p_{1}-\gamma / 3$; see [8]. Using this fact and noticing that $I_{\gamma}$ is a positive operator, by some computation similar to the proof of Theorem 2.6, we complete the proof of Theorem 5.4. We leave the details to the reader.

Finally, we point out that using some discrete Calderón reproducing formulae as in Theorem 1.8 in [3], we can develop a theory of Besov spaces $\dot{B}_{p q}^{s}\left(\mathbb{R}^{2}\right)$ and TriebelLizorkin spaces $\dot{F}_{p q}^{s}\left(\mathbb{R}^{2}\right)$ for full $s \in \mathbb{R}$ and $p, q \in(0, \infty]$. However, to limit the length of this paper and to simplify its presentation, we restrict ourself to the current case.

Acknowledgements. This paper was initially written during the period when Professor Yongsheng Han from Auburn University visited Beijing Normal University in 2005. The author sincerely thanks Professor Yongsheng Han to show him many ideas which are now presented in [3] and to have many stimulating conversations on this subject at that period. He also thanks editor Jose L. Gámez for his careful reading of the manuscript and valuable remarks which made this article more readable.

\section{References}

[1] C. Fefferman and E. M. Stein, Some maximal inequalities, Amer. J. Math. 93 (1971), 107-115.

[2] M. Frazier, B. Jawerth, and G. Weiss, Littlewood-Paley theory and the study of function spaces, CBMS Regional Conference Series in Mathematics, vol. 79, American Mathematical Society, Providence, RI, 1991.

[3] Y. Han and G. Lu, Discrete Littlewood-Paley-Stein theory and multi-parameter Hardy spaces associated with flag singular integrals, available at http://arxiv.org/abs/0801.1701v1.

[4] Y. S. Han and E. T. Sawyer, Littlewood-Paley theory on spaces of homogeneous type and the classical function spaces, Mem. Amer. Math. Soc. 110 (1994), no. 530, 1-126.

[5] D. Müller, F. Ricci, and E. M. Stein, Marcinkiewicz multipliers and multi-parameter structure on Heisenberg(-type) groups, I, Invent. Math. 119 (1995), no. 2, 199-233.

[6] A. Nagel, F. Ricci, and E. M. Stein, Singular integrals with flag kernels and analysis on quadratic CR manifolds, J. Funct. Anal. 181 (2001), no. 1, 29-118.

[7] H.-J. Schmeisser and H. Triebel, Topics in Fourier analysis and function spaces, A WileyInterscience Publication, John Wiley \& Sons Ltd., Chichester, 1987.

[8] E. M. Stein, Singular integrals and differentiability properties of functions, Princeton Mathematical Series, vol. 30, Princeton University Press, Princeton, N.J., 1970.

[9] H. Triebel, Theory of function spaces, Monographs in Mathematics, vol. 78, Birkhäuser Verlag, Basel, 1983.

[10] _ Spaces of Besov-Hardy-Sobolev type, Teubner-Texte zur Mathematik, vol. 15, BSB B. G. Teubner Verlagsgesellschaft, Leipzig, 1978.

[11] _ The structure of functions, Monographs in Mathematics, vol. 97, Birkhäuser Verlag, Basel, 2001. 